LA-13814-PR

Progress Report

Approved for public release;

distribution is unlimited.

\title{
Surface Water Data at \\ Los Alamos National Laboratory: \\ 2000 Water Year
}

\section{Los Alamos \\ NATIONAL LABORATORY}

Los Alamos National Laboratory is operated by the University of California for the United States Department of Energy under contract W-7405-ENG-36. 
The four most recent reports in this classified series are LA-13234-PR, $L A-13403-P R, L A-13551-P R$, and LA-13706-PR.

Edited by Faith Harp, Group IM-1

Photocomposition by Julie Medina, Comforce, for Group IM-1

An Affirmative Action/Equal Opportunity Employer

This report was prepared as an account of work sponsored by an agency of the United States Government. Neither The Regents of the University of California, the United States Government nor any agency thereof, nor any of their employees, makes any warranty, express or implied, or assumes any legal liability or responsibility for the accuracy, completeness, or usefulness of any information, apparatus, product, or process disclosed, or represents that its use would not infringe privately owned rights. Reference herein to any specific commercial product, process, or service by trade name, trademark, manufacturer, or otherwise, does not necessarily constitute or imply its endorsement, recommendation, or favoring by The Regents of the University of California, the United States Government, or any agency thereof. The views and opinions of authors expressed herein do not necessarily state or reflect those of The Regents of the University of California, the United States Government, or any agency thereof. Los Alamos National Laboratory strongly supports academic freedom and a researcher's right to publish; as an institution, however, the Laboratory does not endorse the viewpoint of a publication or guarantee its technical correctness. 
Surface Water Data at

Los Alamos National Laboratory:

2000 Water Year

D. A. Shaull

M. R. Alexander

R. P. Reynolds

R. P. Romero

E. T. Riebsomer

C. T. McLean 



\section{Contents}

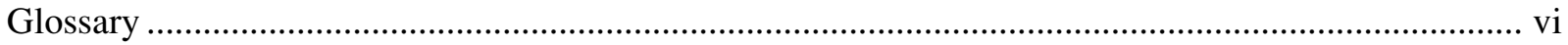

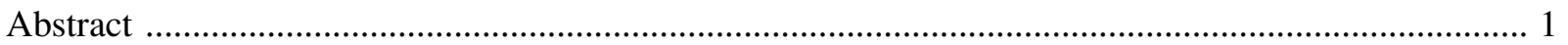

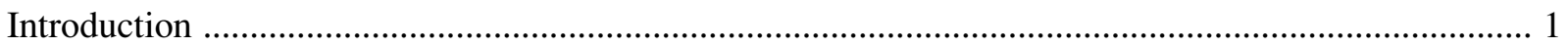

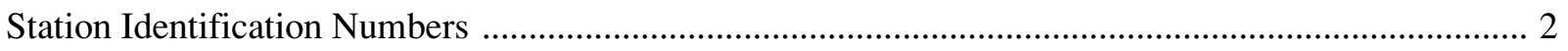

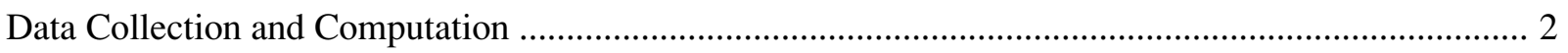

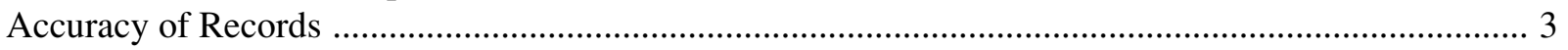

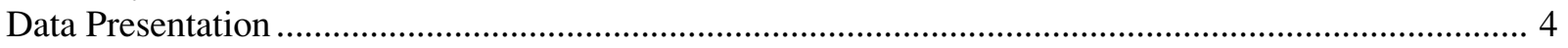

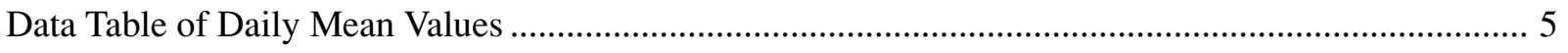

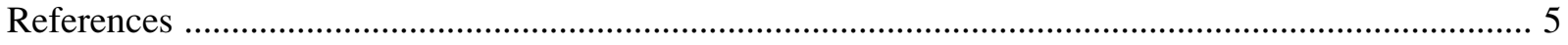

Summary of Selected Peak Flows after the Cerro Grande Fire .............................................................. 6

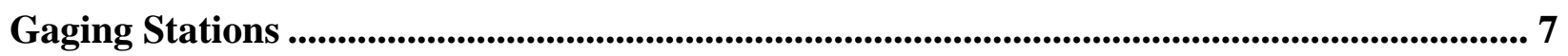

Gaging Stations at Los Alamos National Laboratory ..................................................................... 9

Summary of Discharges from Stream-Monitoring Stations at Los Alamos

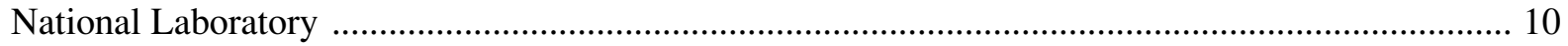

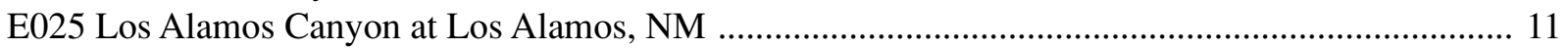

E030 Los Alamos Canyon below Laboratory Technical Area (TA) 2 near

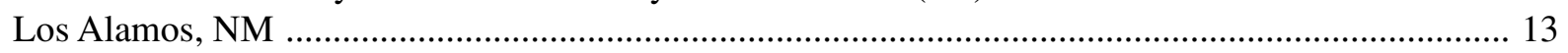

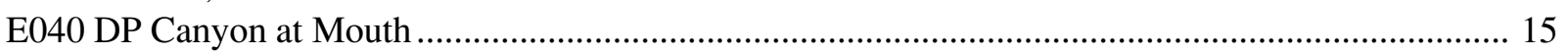

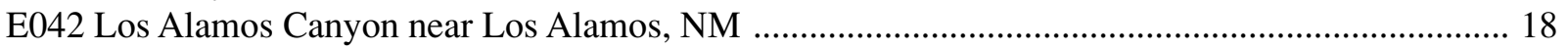

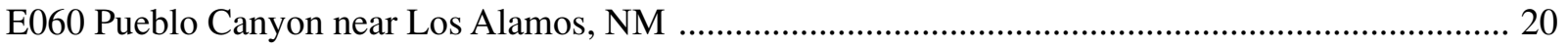

E125 Sandia Canyon above Highway 4 near White Rock, NM ....................................................... 22

E200 Mortandad Canyon at TA-50 near Los Alamos, NM ........................................................... 24

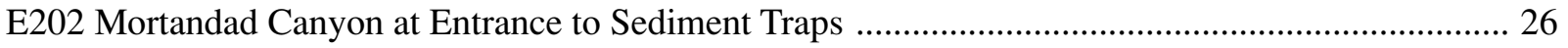

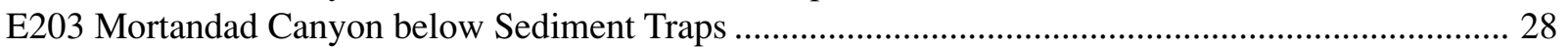

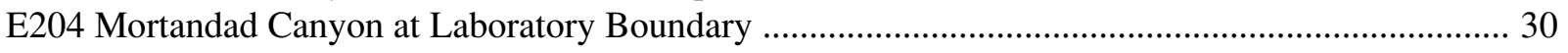

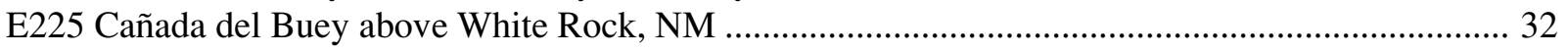

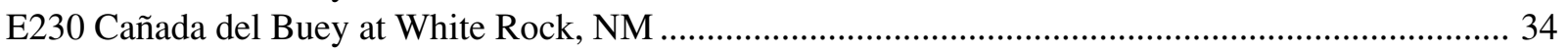

E240 Pajarito Canyon above Highway 501 near Los Alamos, NM ............................................... 36

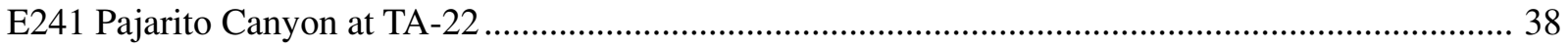

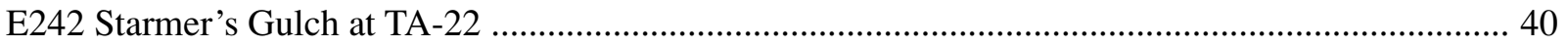

E245 Pajarito Canyon above TA-18 near Los Alamos, NM …......................................................... 43

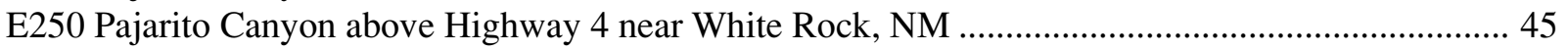

E252 Water Canyon above Highway 501 near Los Alamos, NM .................................................... 47

E253 Cañon del Valle above Highway 501near Los Alamos, NM .................................................... 49

E263 Water Canyon at Highway 4 near White Rock, NM .................................................................. 51

E265 Water Canyon below Highway 4 near White Rock, NM ........................................................... 54

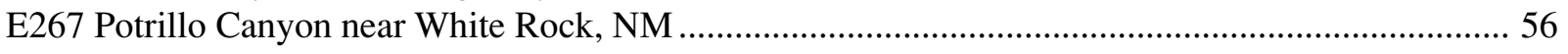

E275 Ancho Canyon near Bandelier National Monument, NM ...................................................... 58

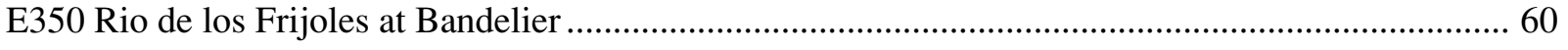

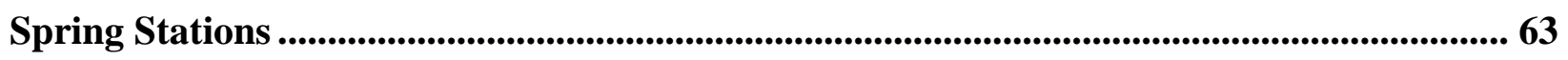

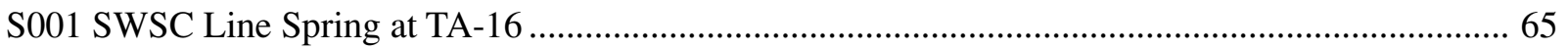

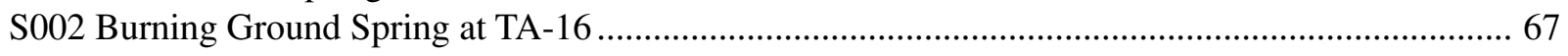

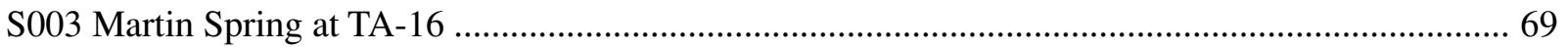




\section{Glossary}

Acre-foot (Ac-Ft, acre-ft) is the quantity of water required to cover 1 acre to a depth of 1 foot and is equivalent to 43,560 cubic feet, 325,851 gallons, or 1233.49 cubic meters.

Cfs-day is the volume of water represented by the flow of 1 cubic foot per second for 24 hours. It is equivalent to 86,400 cubic feet, 1.98347 acre-feet, 646,317 gallons, or 2445 cubic meters.

Control designates a feature downstream from the gage that determines the stage-discharge relation at the gage. This feature may be a natural constriction of the channel, an artificial structure, or a uniform cross section over a long reach of the channel.

Control structure as used in this report is a structure on a stream or canal that is used to regulate the flow or stage of the stream or to prevent the intrusion of salt water.

Cubic feet per second per square mile $\left[\left(\mathrm{ft}^{3} / \mathrm{s}\right) / \mathrm{mi}^{2}\right]$ is the average number of cubic feet of water flowing per second from each square mile of area drained, assuming that the runoff is distributed uniformly in time and area.

Cubic foot per second $\left(\mathrm{ft}^{3} / \mathrm{s}\right)$ is the rate of discharge representing a volume of 1 cubic foot passing a given point during 1 second and is equivalent to 7.48 gallons per second, 448.8 gallons per minute, or 0.02832 cubic meters per second.

Discharge is the volume of water (or more broadly, volume of fluid including suspended sediment) that passes a given point within a given period of time.

Drainage area (DA) of a stream at a specified location is that area, measured in a horizontal plane, enclosed by a topographic divide, from which direct surface runoff from precipitation normally drains by gravity into the stream above the specified point. Figures of drainage area given herein include all closed basins, or noncontributing areas, within the area unless otherwise noted.

Drainage basin is a part of the surface of the earth that is occupied by a drainage system, which consists of a surface stream or a body of impounded surface water together with all tributary surface streams and bodies of impounded surface water.

Gage height $(\mathrm{GH})$ is the water-surface elevation referred to in some arbitrary gage data. Gage height is often used interchangeably with the more general term "stage," although gage height is more appropriate when used with a reading on a gage.

Gaging station is a particular site on a stream, canal, lake, or reservoir where systematic observations of hydrologic data are obtained.

GPS is an abbreviation for Global Positioning System. 
HWM is an abbreviation for high-water mark.

Instantaneous discharge is the discharge at a particular instant of time.

LANL is the acronym for Los Alamos National Laboratory.

Mean discharge (MEAN) is the arithmetic mean of individual daily mean discharges during a specific period.

National Geodetic Vertical Datum of $1929(N G V D)$ is a geodetic datum derived from a general adjustment of the first order level nets of both the United States and Canada. It was formerly called Sea Level Datum of 1929 or "mean sea level" in this series of reports. Although the datum was derived from the average sea level over a period of many years at 26 tide stations along the Atlantic, Gulf of Mexico, and Pacific coasts, it does not necessarily represent the local mean sea level at any particular place.

NPDES is the abbreviation for National Pollution Discharge Elimination System.

SWSC is an abbreviation for sanitary wastewater systems consolidation.

USGS is the abbreviation for United States Geological Survey.

Water year in reports dealing with surface water supply is the 12-month period, October 1 through September 30. The water year is designated by the calendar year in which it ends and which includes 9 of the 12 months. Thus, the year ending September 30, 1980, is called the "1980 water year."

WDR is an abbreviation for "Water-Data Report" in the "Revised Records" paragraph to refer to annual hydrologic-data reports.

WSP is an abbreviation for "Water-Supply Paper" in references to previously published reports. 



\title{
Surface Water Data at Los Alamos National Laboratory: 2000 Water Year
}

\author{
by \\ D. A. Shaull, M. R. Alexander, R. P. Reynolds, R. P. Romero, \\ E. T. Riebsomer, and C. T. McLean
}

\begin{abstract}
The principal investigators collected and computed surface water discharge data from 23 stream-gaging stations that cover most of Los Alamos National Laboratory and one at Bandelier National Monument. Also included are discharge data from three springs, two that flow into Cañon del Valle and one that flows into Water Canyon.

\section{Introduction}

This annual water data report from Los Alamos National Laboratory (LANL) contains flow data from 23 stream-gaging stations that cover most of the Laboratory's property. We focused data collection on the Laboratory's downstream boundary, approximated by New Mexico State Highway 4; the upstream boundary is approximated by New Mexico State Highway 501. Some of the gaging stations are within Laboratory boundaries and were originally installed to assist groups other than the Water Quality and Hydrology Group (ESH-18) that also conduct sitespecific earth science research.
\end{abstract}

This network was severely affected this year by the Cerro Grande Fire, which began on May 5, 2000 , and continued through the month. Twenty stations were damaged to varying degrees by the fire.

After the fire was controlled, the threat of flood was imminent. This report contains a special summary of selected peak flows this year. These could have been more numerous, but rainfall was not particularly intense. The floods reported here were also responsible for destroying eight gaging stations to an extent that no record was collected after the June 28 event. One station (E2455) was removed from this publication because of this event. Others will show no discharge records for the last three months of the water year. These stations are being reinstalled as this document is being prepared. Some of the badly damaged or destroyed stations were in the "new" group not yet published; therefore, the stations missing from publication are limited to E2455.

References to automatic samplers have been removed from "Remarks" in station manuscripts because all stations now have automatic samplers.

Water chemistry data from selected storm events occurring at some stations will be published in the 2000 "Los Alamos National Laboratory Surveillance Report." 


\section{Station Identification Numbers}

The US Geological Survey (USGS), Water Resources Division, assigns a unique identification number to each stream-gaging station it establishes. All sites numbered since 1950 are part of the downstream order system. The downstream order system increases station numbers in the downstream direction along main streams, and, in the case of this report, their respective mouths to the Rio Grande.

This report adheres to the USGS convention of downstream order. Because of the close proximity of stations in this network, the first five digits of all station numbers are 08313 . We have replaced this number string with the letter $\mathrm{E}$ in the station number partly to abbreviate and also to accommodate instrumentation.

\section{Data Collection and Computation}

A complete record-gaging station gathers records of stage and discharge measurements from streams or canals. In addition to gathering these stage and discharge measurements, we directly observe factors affecting the stage/discharge relation, consult weather records, and use other information that supplements base data in determining daily flow. Direct readings on a nonrecording gage or from the data logger provide continuous records of stage. We measure discharge with current meters, using methods adapted by the USGS as a result of experience accumulated since 1880. Standard textbooks describe these methods, as do Water-Supply Paper 2175 and the US Geological Survey Technique of Water Resources Investigations, Book 3, Chapter A6.

We use stage/discharge relation curves to prepare rating tables that give the discharge for any stage measured at a stream-gaging station. When it is necessary to define discharge extremes outside the range of current meter measurements, we extend the curves using

- logarithmic plotting;

- velocity area studies;

- results of indirect measurements of peak discharge, such as slope area or contracted opening measurements and computations of flow over dams or weirs; or

- $\quad$ step backwater techniques.

Daily mean stages (gage heights) are applied to the stage-discharge curves or tables to compute daily mean discharges. If the stage/discharge relation is subject to change because of frequent or continual change in the physical features that form the control, the daily mean discharge is computed by the shifting-control method. In the shifting-control method, correction factors based on individual discharge measurements and notes by personnel taking the measurements are applied to the gage heights before discharges are determined from the curves or tables. 
The shifting-control method is also used if the stage/discharge relation for a station is temporarily changed by the presence of aquatic growth or debris on the control. At some northern stream-gaging stations, the stage/discharge relation is affected by ice in the winter, and it becomes impossible to compute discharge in the usual manner. Discharge for the period of ice effect is computed on the basis of gage height record and occasional winter discharge measurements. Consideration is given to the available information about temperature and precipitation, notes of observations, and comparable discharge records for other stations in the same or nearby basins for comparable periods of time.

For some gaging stations, periods occur when no gage height record is obtained, or the recorded gage height is so faulty that it cannot be used to compute daily discharge or contents. This happens when the recorder stops or otherwise fails to operate properly, intakes are plugged, the float is frozen in the well, etc. For such periods, the daily discharges are estimated on the basis of recorded range-in-stage, prior and subsequent records, discharge measurements, weather records, and record comparison made against other stations in the same or nearby basins. Likewise, daily contents may be estimated from operator logs, prior and subsequent records, inflow-outflow studies, and other information.

\section{Accuracy of Records}

Two factors determine the accuracy of streamflow records:

- stability of the stage-discharge relation or, if the control is unstable, the frequency of discharge measurements; and

- accuracy of measurements or stage, accuracy of discharge measurements, and interpretations of records.

Accuracy attributed to records is noted under "Remarks."

- Excellent-95\% of daily discharges are within 5\% of true value;

- Good-95\% of daily discharges are within $10 \%$ of true value;

- Fair-95\% of daily discharges are within $15 \%$ of true value; and

- Poor-records do not meet the criteria mentioned.

Differences in accuracy may be attributed to different parts of a given record.

The number of significant figures used to report daily mean discharges is based solely on the magnitude of the discharge value:

\begin{tabular}{|l|l|}
\hline \multicolumn{1}{|c|}{ If-the value $\left(\mathrm{ft}^{3} / \mathrm{s}\right)$ is } & \multicolumn{1}{c|}{ Then-it is reported to } \\
\hline less than $1 \mathrm{ft}^{3} / \mathrm{s}$ & nearest hundredth \\
\hline $1-10 \mathrm{ft}^{3} / \mathrm{s}$ & nearest tenth \\
\hline $10-1000 \mathrm{ft}^{3} / \mathrm{s}$ & whole number \\
\hline above $1000 \mathrm{ft}^{3} / \mathrm{s}$ & three significant figures \\
\hline
\end{tabular}




\section{Data Presentation}

The records published in this report are for each gaging station and comprise two parts:

- station manuscript description with photo and

- data table for the water year (October 1, 1999, to September 30, 2000).

The station manuscript provides data under various headings: station location, period of record, average discharge, historical extremes, record accuracy, and other points pertinent to station operation and regulation. Each continuous record of discharge includes the following categories of descriptions.

Location. The most accurate and available maps provide location information. The location of the gage with respect to the vicinity's cultural and physical features is given, as well as a name that refers to place. For a few stations, the US Army Corps of Engineers or the Water Resources Council (River Mileage Measurement, Bulletin 14, rev. October 1968) provided river mileage. We define left and right banks from the perspective of facing downstream.

Drainage Area. The most accurate and available maps provide drainage area measurements. The accuracy of drainage area measurements varies, depending on the type of map available for this purpose.

Period of Record. The period of record is the time during which published records exist for a station or its equivalent station. An equivalent station is one that was in operation at a time that the present station was not and was located so that records from it can reasonably be considered equivalent to records from the present station.

Gage. This section describes the type of gage in current use. The datum of the current gage referred to in the National Geodetic Vertical Datum of 1929 (see Glossary) and a condensed history of the types, locations, and data of previous gages are given under this heading.

Remarks. The date in the station description for water discharge records identifies all periods of estimated daily discharge records. The text also presents information relative to the accuracy of the records, special methods of computation, conditions that affect natural flow at the station, and other pertinent information.

Extremes for Period of Record. Extremes may include maximum and minimum stages and maximum and minimum discharges or content. Unless otherwise qualified, the maximum discharge or content is the instantaneous maximum corresponding to the highest stage that occurred. The highest stage may have been obtained from a graphic or digital recorder, a crest stage gage, or by direct observation of a nonrecording gage. If the maximum stage did not occur on the same day as the maximum discharge or content, it is given separately. Similarly, the minimum is the instantaneous minimum discharge, unless otherwise qualified, and was determined and is reported in the same manner as the maximum. 
Extremes Outside Period of Record. This section contains information concerning major floods or unusually low flows that occurred outside the stated period of record. The information may have been obtained from other agencies, old data files, newspapers, or local citizens.

Extremes for Current Year. Extremes given here are similar to those for the period of record. The time for occurrence of peaks is expressed in 24-hour local standard time. For example, 12:30 A.M. is 0030 and 1:30 P.M. is 1330 . The minimum for the current water year appears in this section.

\section{Data Table of Daily Mean Values}

The daily table of discharge records for stream gaging stations gives the mean discharge for each day of the water year. In the monthly summary for the table, the line headed "Total" gives the sum of the daily figures for each month; the line headed "Mean" gives the average flow in cubic feet per second for the month; and the lines headed "Max" and "Min" give the maximum and minimum daily mean discharges for each month and in acre feet, respectively, in the line headed "Ac-Ft."

\section{References}

Water-Supply Paper 2175 and the US Geological Survey Technique of Water Resources Investigations, Book 3, Chapter A6.

US Army Corps of Engineers, River Mileage Measurement, Bulletin 14, rev. October 1968.

National Geodetic Vertical Datum of 1929.

Previous Los Alamos National Laboratory reports in this series— "Surface Water Date at Los Alamos National Laboratory" for water years 1995-1999

1995: LA-13177-PR (August 1996)

1996: LA-13234-PR (November 1996)

1997: LA-13403-PR (January 1996)

1998: LA-13551-PR (February 1999)

1999: LA-13706-PR (April 2000) 
Summary of Selected Peak Flows after the Cerro Grande Fire

\begin{tabular}{llccc}
$\begin{array}{l}\text { Station } \\
\text { Number }\end{array}$ & Stream & $\begin{array}{c}\text { Drainage Area } \\
\left(\mathbf{m i}^{2}\right)\end{array}$ & $\begin{array}{c}\text { Gage Height } \\
(\mathbf{f t})\end{array}$ & $\begin{array}{c}\text { Discharge } \\
\left(\mathbf{f t}^{3} \mathbf{s} \mathbf{s}\right)\end{array}$ \\
\hline E240 & $\begin{array}{l}\text { Pajarito Canyon } \\
\text { above Highway 501 }\end{array}$ & 1.90 & - & 1,020 \\
E241 & $\begin{array}{l}\text { Pajarito Canyon } \\
\text { at TA-22 }\end{array}$ & 3.97 & 5.00 & 300 \\
E242 & $\begin{array}{l}\text { Starmer's Gulch } \\
\text { at TA-22 }\end{array}$ & 0.82 & 2.75 & 180 \\
E245 & $\begin{array}{l}\text { Pajarito Canyon } \\
\text { above TA-18 }\end{array}$ & 7.84 & 5.03 & 517 \\
E252 & $\begin{array}{l}\text { Water Canyon } \\
\text { above Highway 501 }\end{array}$ & 3.39 & - & 840 \\
E253 & $\begin{array}{l}\text { Cañon del Valle } \\
\text { above Highway 501 }\end{array}$ & 2.46 & - & 740 \\
E263 & $\begin{array}{l}\text { Water Canyon } \\
\text { at Highway 4 }\end{array}$ & 8.20 & 3.78 & 305 \\
E265 & $\begin{array}{l}\text { Water Canyon } \\
\text { below Highway 4 }\end{array}$ & 8.7 & 5.13 & 274 \\
\hline
\end{tabular}

List of peak flows from the following ungaged sites:

1. Rendija Canyon above confluence with Guaje Canyon $\quad D A=9.58 \mathrm{mi}^{2}$

2. Guaje Canyon above mouth of Rendija Canyon $\quad D A=14.6 \mathrm{mi}^{2}$

\begin{tabular}{|c|c|c|c|}
\hline Station & Date & Discharge in $\mathrm{ft}^{\mathbf{t}} / \mathrm{s}$ & Notes \\
\hline \multirow[t]{4}{*}{ No. 1} & July 16 & 900 & \multirow{4}{*}{$\begin{array}{l}\text { All these peaks were less than } 0.15 \\
\text { difference in stage. Discharge was } \\
\text { measured by indirect methods exce } \\
\text { Sept. } 8 \text {, which was estimated. }\end{array}$} \\
\hline & July 17 & 900 & \\
\hline & August 3 & 900 & \\
\hline & September 8 & 900 & \\
\hline \multirow[t]{4}{*}{ No. 2} & July 16 & 840 & \multirow{4}{*}{$\begin{array}{l}\text { Sept. } 8 \text { discharge was estimated on } \\
\text { the basis of HWM compared with } \\
\text { indirectly measured discharge on } \\
\text { Aug. } 27 \text { peak. }\end{array}$} \\
\hline & July 17 & 827 & \\
\hline & August 27 & 1,350 & \\
\hline & September 8 & 1,200 & \\
\hline
\end{tabular}




\section{Gaging Stations}





\section{Gaging Stations at Los Alamos National Laboratory}

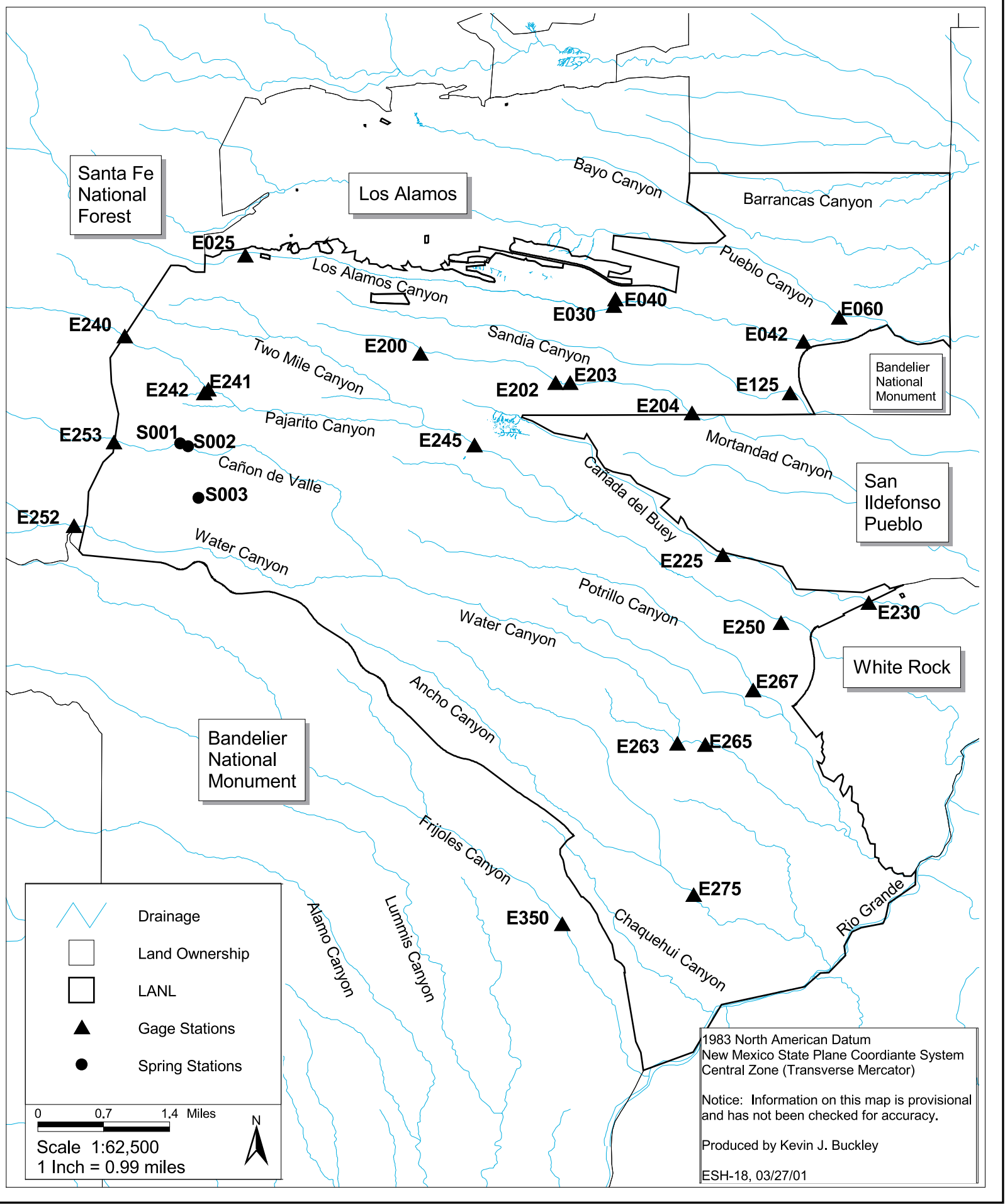




\section{Summary of Discharges from Stream-Monitoring Stations at Los Alamos National Laboratory}

Water Year 2000

October 1, 1999-September 30, 2000

\begin{tabular}{|c|c|c|c|}
\hline Canyon Sites & $\begin{array}{c}\text { Days with } \\
\text { Flow } \\
\end{array}$ & $\begin{array}{c}\text { Volume in } \\
\text { Ac-Ft }\end{array}$ & $\begin{array}{c}\text { Instantaneous } \\
\text { Max in } \mathrm{ft}^{3} / \mathrm{s} \\
\end{array}$ \\
\hline E025 Upper Los Alamos & 53 & 97 & 60 \\
\hline E030 Middle Los Alamos & 21 & 35 & 13 \\
\hline E040 DP Canyon at Mouth & 14 & 30 & 117 \\
\hline E042 Lower Los Alamos* & 22 & 27 & 17 \\
\hline E060 Pueblo* & 365 & 618 & 114 \\
\hline E125 Sandia* & 0 & 0 & 0 \\
\hline E200 Middle Mortandad & 249 & 17 & 12 \\
\hline E202 Mortandad, above Sediment Traps & 3 & 0.4 & 1.6 \\
\hline E203 Mortandad, below Sediment Traps & 0 & 0 & 0 \\
\hline E204 Lower Mortandad* & 0 & 0 & 0 \\
\hline E225 Upper Cañada del Buey & 0 & 0 & 0 \\
\hline E230 Lower Cañada del Buey* & 5 & 2.6 & 33 \\
\hline E240 Upper Pajarito** & 25 & 57 & 1,020 \\
\hline E241 Pajarito at TA-22 & 276 & 95 & 300 \\
\hline E242 Starmer's Gulch at TA-22 & 365 & 46 & 180 \\
\hline E245 Middle Pajarito & 8 & 20 & 517 \\
\hline E250 Lower Pajarito* & 2 & 3.0 & 14 \\
\hline E252 Upper Water** & 273 & 66 & 840 \\
\hline E253 Cañon de Valle** & 1 & 52 & 740 \\
\hline E263 Water Canyon at State Rd. 4 & 19 & 61 & 306 \\
\hline E265 Lower Water* & 22 & 53 & 271 \\
\hline E267 Potrillo* & 5 & 0.7 & 37 \\
\hline E275 Ancho* & 6 & 8.6 & 349 \\
\hline E350 Frijoles at Bandelier & 365 & 526 & 40 \\
\hline
\end{tabular}

*Station at downstream Laboratory boundary.

**Based on partial year of record. 


\section{E025 Los Alamos Canyon at Los Alamos, NM}

Location. Lat 35'52'50", long 106 $19^{\prime} 45^{\prime \prime}$, in SE 1/4 SE 1/4 sec. 17, T. 19 N, R. 6 E, Los Alamos County, on right bank $1.5 \mathrm{mi}$ downstream from Los Alamos Reservoir, and 0.4 mi upstream from "Rainbow" bridge on Diamond Drive over Los Alamos Canyon.

Drainage Area. $7.12 \mathrm{mi}^{2}$.

Period of Record. October 1, 1993, through September 30, 2000.

Gage. Data logger with cellular telemetry and 2-ft Parshall Flume. Elevation of gage is 7,237 ft above National Geodetic Vertical Datum of 1929, from GPS survey.

Remarks. Water discharge records good. Flow partially controlled by Los Alamos Reservoir 1.5 mi upstream.

Extremes for Period of Record. Maximum discharge $60 \mathrm{ft}$ 's No flow at times.

Extremes for Current Water Year. Maximum discharge $60 \mathrm{ft}^{3} / \mathrm{s}$ at $1900 \mathrm{hrs}$, July 18, gage height $2.02 \mathrm{ft}$. No flow at times.

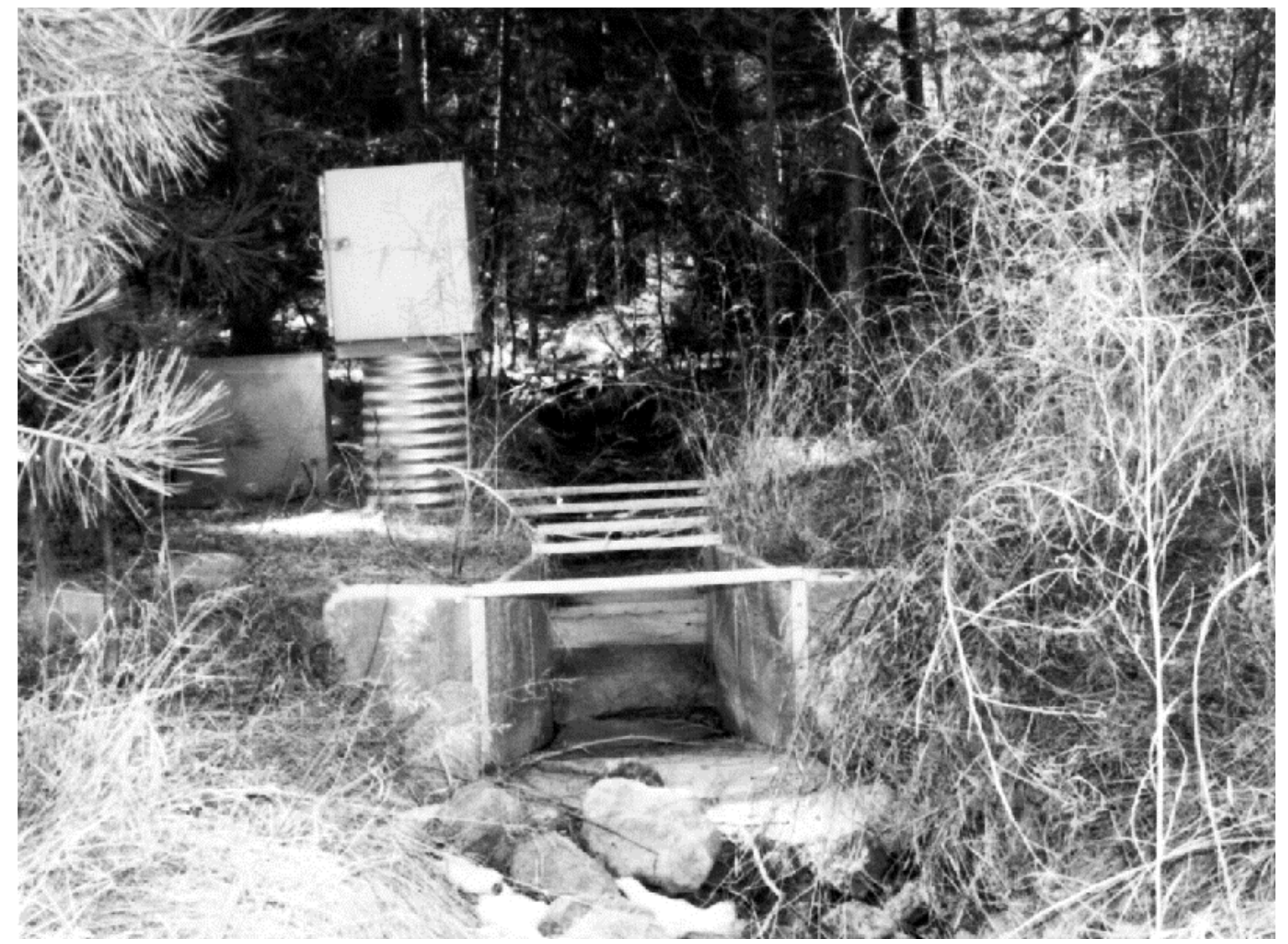




\section{E025 Los Alamos Canyon at Los Alamos, NM}

Daily Mean Discharge in Cubic Feet per Second

Water Year October 1999 to September 2000

\begin{tabular}{|c|c|c|c|c|c|c|c|c|c|c|c|c|}
\hline DAY & OCT & NOV & DEC & JAN & FEB & MAR & APR & MAY & JUN & JUL & AUG & SEP \\
\hline 1 & 0 & 0 & 0 & 0 & 0 & 0 & 0 & 0 & 2.0 & 0 & .02 & 0 \\
\hline 2 & 0 & 0 & 0 & 0 & 0 & 0 & 0 & 0 & 5.4 & 0 & .03 & 0 \\
\hline 3 & 0 & 0 & 0 & 0 & 0 & 0 & 0 & 0 & 4.2 & 0 & .13 & 0 \\
\hline 4 & 0 & 0 & 0 & 0 & 0 & 0 & 0 & 0 & .14 & 0 & .13 & 0 \\
\hline 5 & 0 & 0 & 0 & 0 & 0 & 0 & 0 & 0 & 0 & 0 & .13 & 0 \\
\hline 6 & 0 & 0 & 0 & 0 & 0 & 0 & 0 & 0 & 0 & 0 & .09 & 1.0 \\
\hline 7 & 0 & 0 & 0 & 0 & 0 & 0 & 0 & 0 & 0 & 0 & .07 & 1.8 \\
\hline 8 & 0 & 0 & 0 & 0 & 0 & 0 & 0 & 0 & 0 & 0 & .07 & 1.7 \\
\hline 9 & 0 & 0 & 0 & 0 & 0 & 0 & 0 & 0 & 0 & .50 & .07 & .08 \\
\hline 10 & 0 & 0 & 0 & 0 & 0 & 0 & 0 & 0 & 0 & 0 & .07 & .04 \\
\hline 11 & 0 & 0 & 0 & 0 & 0 & 0 & 0 & 0 & 0 & 0 & .45 & 1.1 \\
\hline 12 & 0 & 0 & 0 & 0 & 0 & 0 & 0 & 0 & 0 & 0 & 0 & 2.1 \\
\hline 13 & 0 & 0 & 0 & 0 & 0 & 0 & 0 & 0 & 0 & 0 & 0 & .82 \\
\hline 14 & 0 & 0 & 0 & 0 & 0 & 0 & 0 & 0 & 0 & 0 & 0 & 1.2 \\
\hline 15 & 0 & 0 & 0 & 0 & 0 & 0 & 0 & 0 & 0 & .01 & 0 & .36 \\
\hline 16 & 0 & 0 & 0 & 0 & 0 & 0 & 0 & 0 & 0 & 0 & 0 & .07 \\
\hline 17 & 0 & 0 & 0 & 0 & 0 & 0 & 0 & 0 & 0 & .02 & 0 & .04 \\
\hline 18 & 0 & 0 & 0 & 0 & 0 & 0 & 0 & 0 & 0 & 2.1 & 0 & .03 \\
\hline 19 & 0 & 0 & 0 & 0 & 0 & 0 & 0 & 0 & 0 & 5.8 & 0 & .10 \\
\hline 20 & 0 & 0 & 0 & 0 & 0 & 0 & 0 & 0 & 0 & 13 & 0 & 0 \\
\hline 21 & 0 & 0 & 0 & 0 & 0 & 0 & 0 & 0 & 0 & 1.9 & 0 & 0 \\
\hline 22 & 0 & 0 & 0 & 0 & 0 & 0 & 0 & 0 & 0 & .13 & 0 & 0 \\
\hline 23 & 0 & 0 & 0 & 0 & 0 & 0 & 0 & 0 & 0 & .09 & 0 & 0 \\
\hline 24 & 0 & 0 & 0 & 0 & 0 & 0 & 0 & 0 & 0 & .07 & 0 & 0 \\
\hline 25 & 0 & 0 & 0 & 0 & 0 & 0 & 0 & .04 & 0 & .07 & 0 & 0 \\
\hline 26 & 0 & 0 & 0 & 0 & 0 & 0 & 0 & .14 & 0 & .07 & 0 & 0 \\
\hline 27 & 0 & 0 & 0 & 0 & 0 & 0 & 0 & .20 & 0 & .07 & 0 & 0 \\
\hline 28 & 0 & 0 & 0 & 0 & 0 & 0 & 0 & .17 & 0 & .07 & 0 & 0 \\
\hline 29 & 0 & 0 & 0 & 0 & ----- & 0 & 0 & .17 & 0 & .07 & 0 & 0 \\
\hline 30 & 0 & 0 & 0 & 0 & ----- & 0 & 0 & .17 & 0 & .07 & 0 & 0 \\
\hline 31 & 0 & ------ & 0 & 0 & ----- & 0 & ----- & .53 & ----- & .07 & 0 & ------ \\
\hline Total & 0 & 0 & 0 & 0 & 0 & 0 & 0 & 1.42 & 11.74 & 24.11 & 1.26 & 10.44 \\
\hline Mean & 0 & 0 & 0 & 0 & 0 & 0 & 0 & .046 & .39 & .78 & .041 & .35 \\
\hline Max & 0 & 0 & 0 & 0 & 0 & 0 & 0 & .53 & 5.4 & 13 & .45 & 2.1 \\
\hline Min & 0 & 0 & 0 & 0 & 0 & 0 & 0 & 0 & 0 & 0 & 0 & 0 \\
\hline Ac-Ft & 0 & 0 & 0 & 0 & 0 & 0 & 0 & 2.8 & 24 & 47 & 2.5 & 21 \\
\hline Wtr Year & 2000 & Total & 48 & & Mean & .13 & Max & 13 & Min & 0 & $\mathrm{Ac}-\mathrm{Ft}$ & 97 \\
\hline Cal Year & 1999 & Total & 30 & & Mean & .084 & Max & 3.1 & Min & 0 & $\mathrm{Ac}-\mathrm{Ft}$ & 61 \\
\hline
\end{tabular}




\section{E030 Los Alamos Canyon below Laboratory Technical Area (TA) 2 near Los Alamos, NM}

Location. Lat $35^{\circ} 52^{\prime} 21^{\prime \prime}$, long $106^{\circ} 15^{\prime} 36^{\prime \prime}$, SW 1/4, SE 1/4 sec. 14, T. 19 N, R. 6 E, Los Alamos County, $150 \mathrm{ft}$ upstream from mouth of DP Canyon wash and 2.4 mi upstream from NM State Highway 4.

Drainage Area. $8.58 \mathrm{mi}^{2}$.

Period of Record. July 1994 to September 30, 2000.

Gage. Data logger with cellular telemetry and concrete control. Elevation of gage is 6,627 ft above National Geodetic Vertical Datum of 1929, from GPS survey.

Remarks. Water discharge records fair. Flow partially regulated by Los Alamos Reservoir about 2.5 mi upstream.

Extremes Outside Period of Record. Flood of July 31, 1968, was $329 \mathrm{ft}^{3} / \mathrm{s}$ from slope area determination. Gage height was established later at $3.71 \mathrm{ft}$ present datum.

Extremes for Period of Record. Maximum discharge $13 \mathrm{ft}^{3} / \mathrm{s}$, July 9, 1999, gage height, $1.60 \mathrm{ft}$. No flow most of time.

Extremes for Current Water Year. Maximum discharge $13 \mathrm{ft}^{3} / \mathrm{s}$ at $1805 \mathrm{hrs}$ June 2, gage height $1.60 \mathrm{ft}$. No flow most of time.

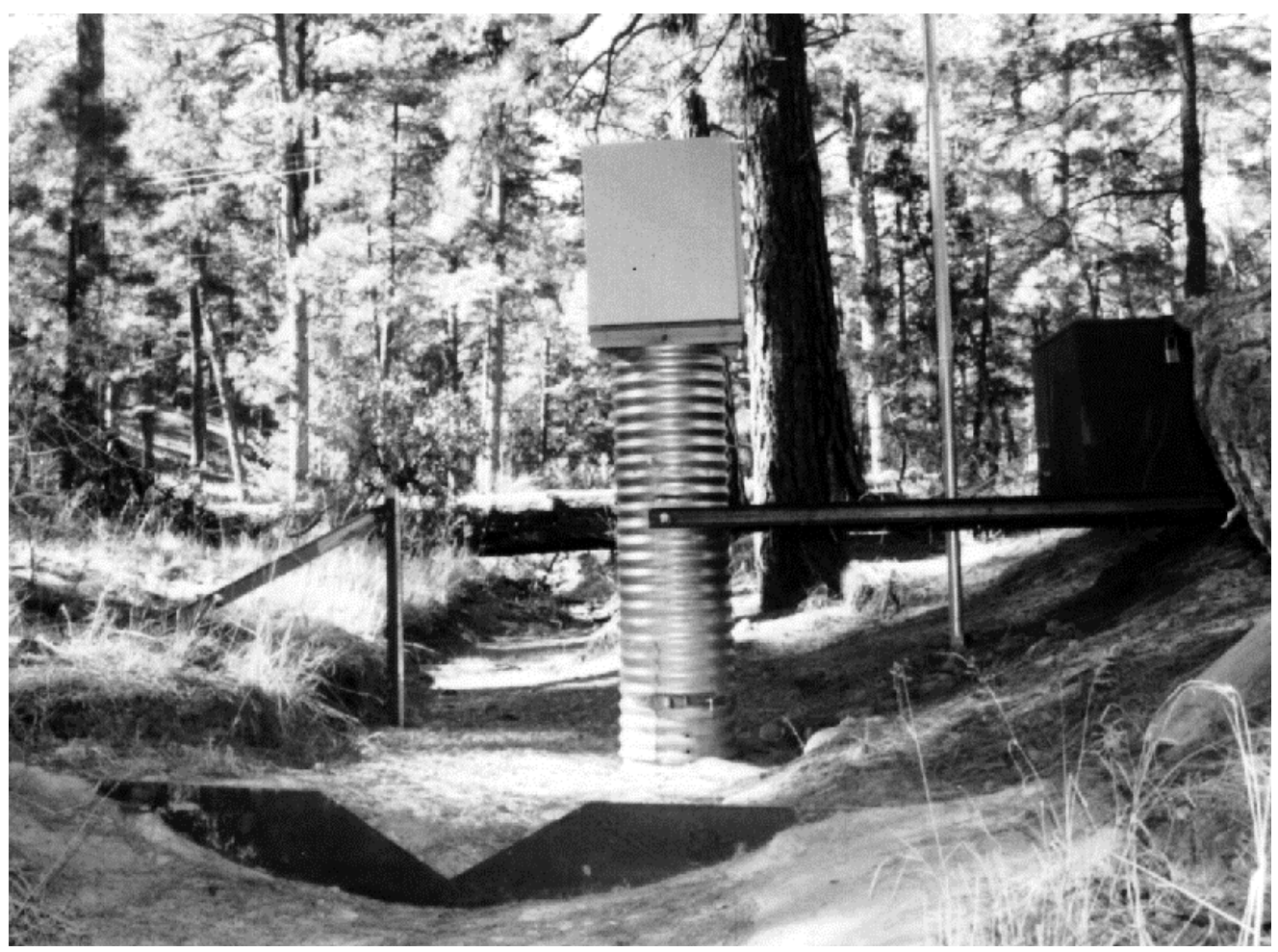




\section{E030 Los Alamos Canyon below TA-2 near Los Alamos, NM}

Daily Mean Discharge in Cubic Feet per Second

Water Year October 1999 to September 2000

\begin{tabular}{|c|c|c|c|c|c|c|c|c|c|c|c|c|}
\hline DAY & OCT & NOV & DEC & JAN & FEB & MAR & APR & MAY & JUN & JUL & AUG & SEP \\
\hline 1 & 0 & 0 & 0 & 0 & 0 & 0 & 0 & 0 & 0 & 0 & $0^{*}$ & 0 \\
\hline 2 & 0 & 0 & 0 & 0 & 0 & 0 & 0 & 0 & 2.3 & 0 & $0^{*}$ & 0 \\
\hline 3 & 0 & 0 & 0 & 0 & 0 & 0 & 0 & 0 & 3.9 & 0 & $0^{*}$ & 0 \\
\hline 4 & 0 & 0 & 0 & 0 & 0 & 0 & 0 & 0 & 1.1 & 0 & $0^{*}$ & 0 \\
\hline 5 & 0 & 0 & 0 & 0 & 0 & 0 & 0 & 0 & .27 & 0 & $0^{*}$ & 0 \\
\hline 6 & 0 & 0 & 0 & 0 & 0 & 0 & 0 & 0 & .13 & 0 & $0^{*}$ & 0 \\
\hline 7 & 0 & 0 & 0 & 0 & 0 & 0 & 0 & 0 & 0 & 0 & $0^{*}$ & .02 \\
\hline 8 & 0 & 0 & 0 & 0 & 0 & 0 & 0 & 0 & 0 & 0 & $0^{*}$ & .35 \\
\hline 9 & 0 & 0 & 0 & 0 & 0 & 0 & 0 & 0 & .18 & .38 & $0^{*}$ & .10 \\
\hline 10 & 0 & 0 & 0 & 0 & 0 & 0 & 0 & 0 & 0 & .28 & $0^{*}$ & 0 \\
\hline 11 & 0 & 0 & 0 & 0 & 0 & 0 & 0 & 0 & 0 & .10 & $0^{*}$ & 0 \\
\hline 12 & 0 & 0 & 0 & 0 & 0 & 0 & 0 & 0 & 0 & 0 & $0^{*}$ & 0 \\
\hline 13 & 0 & 0 & 0 & 0 & 0 & 0 & 0 & 0 & 0 & 0 & $0^{*}$ & .41 \\
\hline 14 & 0 & 0 & 0 & 0 & 0 & 0 & 0 & 0 & 0 & 0 & $0^{*}$ & 1.5 \\
\hline 15 & 0 & 0 & 0 & 0 & 0 & 0 & 0 & 0 & 0 & 0 & $0^{*}$ & .74 \\
\hline 16 & 0 & 0 & 0 & 0 & 0 & 0 & 0 & 0 & 0 & 0 & $0^{*}$ & .01 \\
\hline 17 & 0 & 0 & 0 & 0 & 0 & 0 & 0 & 0 & 0 & 0 & $0^{*}$ & 0 \\
\hline 18 & 0 & 0 & 0 & 0 & 0 & 0 & 0 & 0 & 0 & .06 & $0^{*}$ & 0 \\
\hline 19 & 0 & 0 & 0 & 0 & 0 & 0 & 0 & 0 & 0 & 1.2 & $0^{*}$ & 0 \\
\hline 20 & 0 & 0 & 0 & 0 & 0 & 0 & 0 & 0 & 0 & 3.3 & $0^{*}$ & 0 \\
\hline 21 & 0 & 0 & 0 & 0 & 0 & 0 & 0 & 0 & 0 & 1.1 & $0^{*}$ & 0 \\
\hline 22 & 0 & 0 & 0 & 0 & 0 & 0 & 0 & 0 & 0 & .15 & $0^{*}$ & 0 \\
\hline 23 & 0 & 0 & 0 & 0 & 0 & 0 & 0 & 0 & 0 & 0 & 0 & 0 \\
\hline 24 & 0 & 0 & 0 & 0 & 0 & 0 & 0 & 0 & 0 & 0 & 0 & 0 \\
\hline 25 & 0 & 0 & 0 & 0 & 0 & 0 & 0 & 0 & 0 & 0 & 0 & 0 \\
\hline 26 & 0 & 0 & 0 & 0 & 0 & 0 & 0 & 0 & 0 & 0 & 0 & 0 \\
\hline 27 & 0 & 0 & 0 & 0 & 0 & 0 & 0 & 0 & 0 & $0^{*}$ & 0 & 0 \\
\hline 28 & 0 & 0 & 0 & 0 & 0 & 0 & 0 & 0 & 0 & $0^{*}$ & 0 & 0 \\
\hline 29 & 0 & 0 & 0 & 0 & ----- & 0 & 0 & 0 & 0 & $0^{*}$ & 0 & 0 \\
\hline 30 & 0 & 0 & 0 & 0 & ----- & 0 & 0 & 0 & 0 & $0^{*}$ & 0 & 0 \\
\hline 31 & 0 & ---- & 0 & 0 & ----- & 0 & ----- & 0 & ----- & $0^{*}$ & 0 & ---- \\
\hline Total & 0 & 0 & 0 & 0 & 0 & 0 & 0 & 0 & 7.88 & 6.57 & 0 & 3.13 \\
\hline Mean & 0 & 0 & 0 & 0 & 0 & 0 & 0 & 0 & .26 & .21 & 0 & .10 \\
\hline Max & 0 & 0 & 0 & 0 & 0 & 0 & 0 & 0 & 3.9 & 3.3 & 0 & 1.5 \\
\hline Min & 0 & 0 & 0 & 0 & 0 & 0 & 0 & 0 & 0 & 0 & 0 & 0 \\
\hline Ac-Ft & 0 & 0 & 0 & 0 & 0 & 0 & 0 & 0 & 16 & 13 & 0 & 6.2 \\
\hline Wtr Year & 2000 & Total & 17.58 & & lean & .048 & $\operatorname{Max}$ & 3.9 & Min & 0 & $\mathrm{Ac}-\mathrm{Ft}$ & 35 \\
\hline Cal Year & 1999 & Total & 40.45 & & lean & .11 & Max & 2.9 & Min & 0 & $\mathrm{Ac}-\mathrm{Ft}$ & 80 \\
\hline
\end{tabular}

${ }^{\star}$ Estimated. 


\section{E040 DP Canyon at Mouth}

Location. Lat $35^{\circ} 52^{\prime} 24^{\prime \prime}$, long $106^{\circ} 15^{\prime} 34^{\prime \prime}$, SW 1/4 sec. 14, T. 19 N, R. 6 E, Los Alamos County $150 \mathrm{ft}$ upstream from confluence of DP Canyon and Los Alamos Canyon and $2.4 \mathrm{mi}$ upstream from NM State Highway 4.

Drainage Area. $0.57 \mathrm{mi}^{2}$.

Period of Record. May 1999 to September 30, 2000.

Gage. Data logger with cellular telemetry and concrete control. Elevation of gage is 6,625 ft above National Geodetic Vertical Datum of 1929, from GPS survey.

Remarks. Water discharge records poor.

Extremes for Period of Record. Maximum discharge $117 \mathrm{ft}^{3} / \mathrm{s}$ at $1640 \mathrm{hrs}$, July 25, 2000, gage height $3.69 \mathrm{ft}$. No flow most of time.

Extremes for May 1999-September 2000. Maximum discharge $117 \mathrm{ft}^{3} / \mathrm{s}$ at $1640 \mathrm{hrs,} \mathrm{July} \mathrm{25,}$ gage height $3.69 \mathrm{ft}$. No flow most of time.

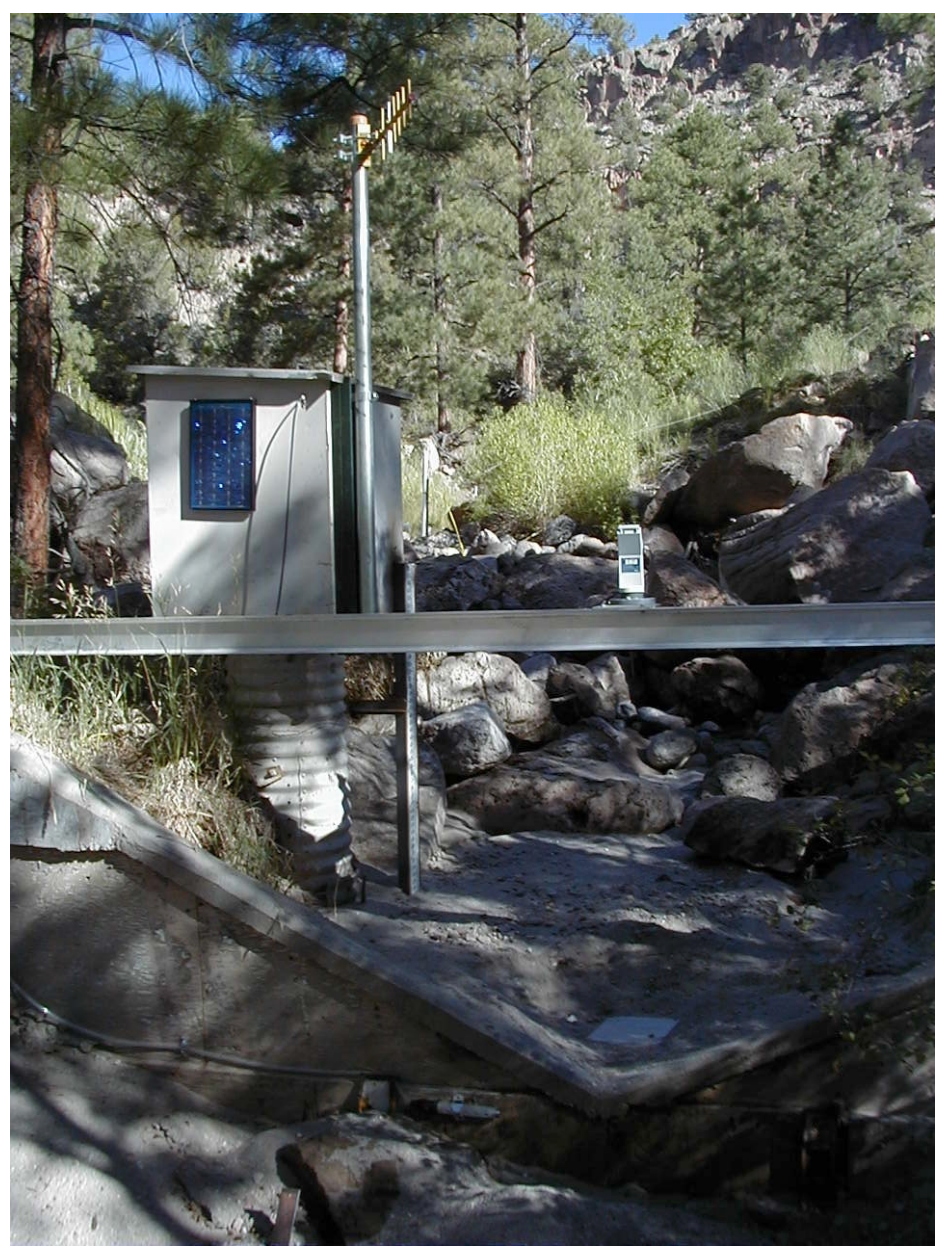




\section{E040 DP Canyon at Mouth}

Daily Mean Discharge in Cubic Feet per Second

Water Year October 1998 to September 1999

\begin{tabular}{|c|c|c|c|c|c|c|c|c|c|c|c|c|}
\hline DAY & OCT & NOV & DEC & JAN & FEB & MAR & APR & MAY & JUN & JUL & AUG & SEP \\
\hline 1 & & & & & & & & & 0 & 0 & .12 & 0 \\
\hline 2 & & & & & & & & & 0 & 0 & .26 & 0 \\
\hline 3 & & & & & & & & & 0 & 0 & .48 & 0 \\
\hline 4 & & & & & & & & & 0 & 0 & .76 & 0 \\
\hline 5 & & & & & & & & & 0 & 0 & 1.1 & 0 \\
\hline 6 & & & & & & & & & 0 & .06 & .37 & .13 \\
\hline 7 & & & & & & & & & 0 & .46 & .43 & 1.8 \\
\hline 8 & & & & & & & & & 0 & 1.5 & .68 & .02 \\
\hline 9 & & & & & & & & & 0 & 2.5 & .40 & 0 \\
\hline 10 & & & & & & & & & 0 & 2.3 & .96 & 0 \\
\hline 11 & & & & & & & & & 0 & 1.8 & 0 & 0 \\
\hline 12 & & & & & & & & & 0 & 0 & 0 & 0 \\
\hline 13 & & & & & & & & & 0 & 0 & 0 & 0 \\
\hline 14 & & & & & & & & & 0 & 0 & 1.0 & .97 \\
\hline 15 & & & & & & & & & 0 & 0 & .20 & .64 \\
\hline 16 & & & & & & & & & .16 & 0 & 0 & 2.8 \\
\hline 17 & & & & & & & & & .43 & 0 & 0 & 1.9 \\
\hline 18 & & & & & & & & & .02 & 0 & 0 & .59 \\
\hline 19 & & & & & & & & 0 & .31 & 0 & 0 & .01 \\
\hline 20 & & & & & & & & 0 & 1.2 & 0 & 0 & 0 \\
\hline 21 & & & & & & & & 0 & 2.0 & 0 & 0 & 0 \\
\hline 22 & & & & & & & & 0 & 1.0 & 0 & 0 & 0 \\
\hline 23 & & & & & & & & 0 & .02 & 0 & 0 & 0 \\
\hline 24 & & & & & & & & .74 & 0 & 0 & 0 & 0 \\
\hline 25 & & & & & & & & .03 & 0 & 0 & 0 & 0 \\
\hline 26 & & & & & & & & 0 & 0 & 0 & 0 & 0 \\
\hline 27 & & & & & & & & .04 & 0 & 0 & .18 & 0 \\
\hline 28 & & & & & & & & .35 & 0 & 0 & 2.8 & 0 \\
\hline 29 & & & & & & & & .73 & 0 & .01 & 9.3 & 0 \\
\hline 30 & & & & & & & & 2.3 & 0 & .06 & 1.0 & 0 \\
\hline 31 & & & & & & & & .08 & ------ & .13 & 0 & ---- \\
\hline Total & & & & & & & & 4.27 & 5.14 & 8.82 & 20.04 & 8.86 \\
\hline Mean & & & & & & & & .33 & .17 & .28 & .65 & .31 \\
\hline Max & & & & & & & & 2.3 & 2.0 & 2.5 & 9.3 & 2.8 \\
\hline Min & & & & & & & & 0 & 0 & 0 & 0 & 0 \\
\hline Ac-Ft & & & & & & & & 8.5 & 10 & 18 & 39 & 18 \\
\hline Wtr Year & 1999 & Total & 47.13 & & Mean & .35 & Max & 9.3 & Min & 0 & $\mathrm{Ac}-\mathrm{Ft}$ & 93 \\
\hline Cal Year & 1998 & Total & 0 & & Mean & 0 & Max & 0 & Min & 0 & $\mathrm{Ac}-\mathrm{Ft}$ & 0 \\
\hline
\end{tabular}


E040 DP Canyon at Mouth

Daily Mean Discharge in Cubic Feet per Second

Water Year October 1999 to September 2000

\begin{tabular}{|c|c|c|c|c|c|c|c|c|c|c|c|c|}
\hline DAY & OCT & NOV & DEC & JAN & FEB & MAR & APR & MAY & JUN & JUL & AUG & SEP \\
\hline 1 & 0 & 0 & 0 & 0 & 0 & 0 & 0 & 0 & 0 & 0 & 0 & 0 \\
\hline 2 & 0 & 0 & 0 & 0 & 0 & 0 & 0 & 0 & 3.9 & 0 & 0 & 0 \\
\hline 3 & 0 & 0 & 0 & 0 & 0 & 0 & 0 & 0 & 0 & 0 & 0 & 0 \\
\hline 4 & 0 & 0 & 0 & 0 & 0 & 0 & 0 & 0 & 0 & 0 & 0 & 0 \\
\hline 5 & 0 & 0 & 0 & 0 & 0 & 0 & 0 & 0 & 0 & 0 & 0 & 0 \\
\hline 6 & 0 & 0 & 0 & 0 & 0 & 0 & 0 & 0 & 0 & 0 & 0 & 0 \\
\hline 7 & 0 & 0 & 0 & 0 & 0 & 0 & 0 & 0 & 0 & 0 & 0 & 0 \\
\hline 8 & 0 & 0 & 0 & 0 & 0 & 0 & 0 & 0 & 0 & 0 & 0 & 0 \\
\hline 9 & 0 & 0 & 0 & 0 & 0 & 0 & 0 & 0 & 0 & .11 & 0 & .02 \\
\hline 10 & 0 & 0 & 0 & 0 & 0 & 0 & 0 & 0 & 0 & 0 & 0 & 0 \\
\hline 11 & 0 & 0 & 0 & 0 & 0 & 0 & 0 & 0 & 0 & 0 & 0 & 0 \\
\hline 12 & 0 & 0 & 0 & 0 & 0 & 0 & .25 & 0 & 0 & 0 & 0 & 0 \\
\hline 13 & 0 & 0 & 0 & 0 & 0 & 0 & 0 & 0 & 0 & 0 & 0 & 0 \\
\hline 14 & 0 & 0 & 0 & 0 & 0 & 0 & 0 & 0 & 0 & 0 & 0 & 0 \\
\hline 15 & 0 & 0 & 0 & 0 & 0 & 0 & 0 & 0 & 0 & 0 & 0 & 0 \\
\hline 16 & 0 & 0 & 0 & 0 & 0 & 0 & 0 & 0 & 0 & .11 & 0 & 0 \\
\hline 17 & 0 & 0 & 0 & 0 & 0 & 0 & 0 & 0 & 0 & .84 & 2.0 & 0 \\
\hline 18 & 0 & 0 & 0 & 0 & 0 & 0 & 0 & 0 & 0 & 3.3 & 1.0 & 0 \\
\hline 19 & 0 & 0 & 0 & 0 & 0 & 0 & 0 & 0 & 0 & 0 & 0 & 0 \\
\hline 20 & 0 & 0 & 0 & 0 & 0 & 0 & 0 & 0 & 0 & 0 & 0 & 0 \\
\hline 21 & 0 & 0 & 0 & 0 & 0 & 0 & 0 & 0 & 0 & 0 & 0 & 0 \\
\hline 22 & 0 & 0 & 0 & 0 & 0 & .38 & 0 & 0 & 0 & 0 & 0 & 0 \\
\hline 23 & 0 & 0 & 0 & 0 & 0 & .01 & 0 & 0 & 0 & 0 & 0 & 0 \\
\hline 24 & 0 & 0 & 0 & 0 & 0 & 0 & 0 & 0 & 0 & 0 & 0 & 0 \\
\hline 25 & 0 & 0 & 0 & 0 & 0 & 0 & 0 & 0 & 0 & 0 & 0 & 0 \\
\hline 26 & 0 & 0 & 0 & 0 & 0 & 0 & 0 & 0 & 0 & 0 & 0 & 0 \\
\hline 27 & 0 & 0 & 0 & 0 & 0 & 0 & 0 & 0 & 0 & 0 & 0 & 0 \\
\hline 28 & 0 & 0 & 0 & 0 & 0 & 0 & 0 & 0 & 0 & 0 & 0 & 0 \\
\hline 29 & 0 & 0 & 0 & 0 & ----- & 0 & 0 & 0 & 0 & .08 & 0 & 0 \\
\hline 30 & 0 & 0 & 0 & 0 & ------ & 0 & 0 & 0 & 0 & 1.7 & 0 & 0 \\
\hline 31 & 0 & ------ & 0 & 0 & ------ & 0 & ----- & 0 & ----- & 1.5 & 0 & ----- \\
\hline Total & 0 & 0 & 0 & 0 & 0 & 0.39 & 0.25 & 0 & 3.9 & 7.64 & 3.0 & 0.02 \\
\hline Mean & 0 & 0 & 0 & 0 & 0 & .013 & .008 & 0 & .13 & .25 & .097 & .001 \\
\hline Max & 0 & 0 & 0 & 0 & 0 & .38 & .25 & 0 & 3.9 & 3.3 & 2.0 & .02 \\
\hline Min & 0 & 0 & 0 & 0 & 0 & 0 & 0 & 0 & 0 & 0 & 0 & 0 \\
\hline Ac-Ft & 0 & 0 & 0 & 0 & 0 & .7 & .5 & 0 & 7.7 & 15 & 6.0 & .04 \\
\hline Wtr Year & 2000 & Total & 15 & & Mean & .042 & Max & 3.9 & Min & 0 & $\mathrm{Ac}-\mathrm{Ft}$ & 30 \\
\hline Cal Year & 1999 & Total & 52. & & Mean & .14 & Max & 10 & Min & 0 & Ac- $\mathrm{Ft}$ & 105 \\
\hline
\end{tabular}




\section{E042 Los Alamos Canyon near Los Alamos, NM}

Location. Lat 35 52'01", long 106¹3'25", in SW 1/4 sec. 20, T. 19 N R. 7 E, Santa Fe County, on right bank, 1/4 mi upstream from NM State Highway 4, 2.7 mi NW of White Rock, NM, $3.9 \mathrm{mi} \mathrm{E}$ of Los Alamos, and $13.5 \mathrm{mi} \mathrm{SW}$ of Española.

Drainage Area. $9.08 \mathrm{mi}^{2}$.

Period of Record. November 1970 to June 1971, October 1991 to September 30, 2000.

Gage. Data logger with cellular telemetry and concrete control. Elevation of gage is 6,383 ft above National Geodetic Vertical Datum of 1929, from GPS survey.

Remarks. Water discharge records fair.

Extremes for Period of Record. Maximum discharge $171 \mathrm{ft}^{3} / \mathrm{s}$, August 22, 1997, gage height $2.95 \mathrm{ft}$. No flow at times.

Extremes for Current Water Year. Maximum discharge $17 \mathrm{ft}^{3} / \mathrm{s}$, at $1850 \mathrm{hrs}$, June 2, gage height $2.10 \mathrm{ft}$. No flow most of time.

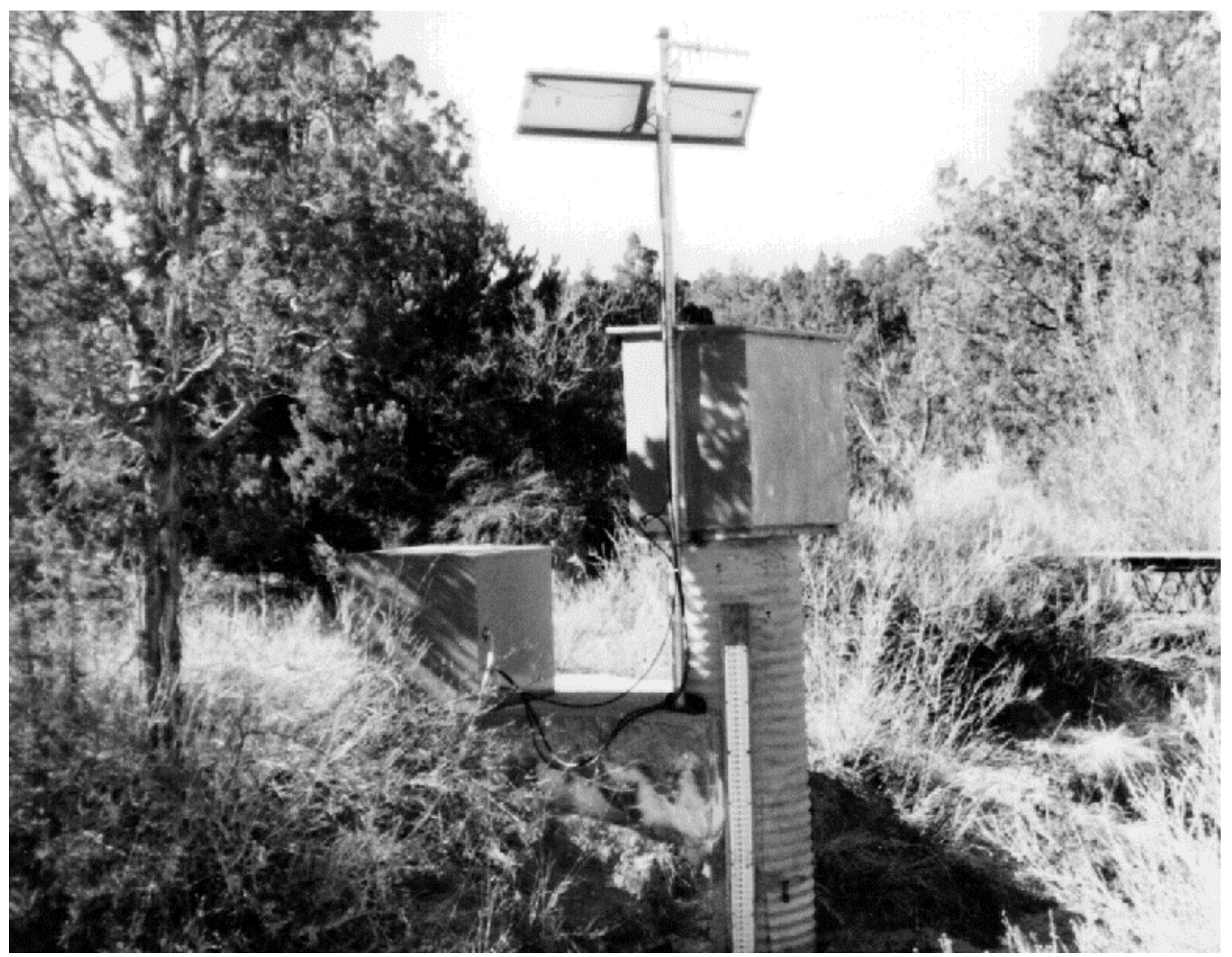




\section{E042 Los Alamos Canyon near Los Alamos, NM}

Daily Mean Discharge in Cubic Feet per Second

Water Year October 1999 to September 2000

\begin{tabular}{|c|c|c|c|c|c|c|c|c|c|c|c|c|}
\hline DAY & ОСТ & NOV & DEC & JAN & FEB & MAR & APR & MAY & JUN & JUL & AUG & SEP \\
\hline 1 & 0 & 0 & 0 & 0 & 0 & 0 & 0 & 0 & 0 & 0 & 0 & 0 \\
\hline 2 & 0 & 0 & 0 & 0 & 0 & 0 & 0 & 0 & 1.1 & 0 & 0 & 0 \\
\hline 3 & 0 & 0 & 0 & 0 & 0 & 0 & 0 & 0 & 1.9 & 0 & 0 & 0 \\
\hline 4 & 0 & 0 & 0 & 0 & 0 & 0 & 0 & 0 & .16 & 0 & 0 & 0 \\
\hline 5 & 0 & 0 & 0 & 0 & 0 & 0 & 0 & 0 & 0 & 0 & 0 & 0 \\
\hline 6 & 0 & 0 & 0 & 0 & 0 & 0 & 0 & 0 & 0 & 0 & 0 & 0 \\
\hline 7 & 0 & 0 & 0 & 0 & 0 & 0 & 0 & 0 & 0 & 0 & 0 & 0 \\
\hline 8 & 0 & 0 & 0 & 0 & 0 & 0 & 0 & 0 & 0 & 0 & 0 & .05 \\
\hline 9 & 0 & 0 & 0 & 0 & 0 & 0 & 0 & 0 & .01 & .06 & .01 & 0 \\
\hline 10 & 0 & 0 & 0 & 0 & 0 & 0 & 0 & 0 & 0 & 0 & 0 & 0 \\
\hline 11 & 0 & 0 & 0 & 0 & 0 & 0 & 0 & 0 & 0 & 0 & 0 & .06 \\
\hline 12 & 0 & 0 & 0 & 0 & 0 & 0 & 0 & 0 & 0 & 0 & 0 & 1.3 \\
\hline 13 & 0 & 0 & 0 & 0 & 0 & 0 & 0 & 0 & 0 & $0^{*}$ & 0 & .01 \\
\hline 14 & 0 & 0 & 0 & 0 & 0 & 0 & 0 & 0 & 0 & $0^{*}$ & 0 & .20 \\
\hline 15 & 0 & 0 & 0 & 0 & 0 & 0 & 0 & 0 & 0 & $0^{*}$ & 0 & 0 \\
\hline 16 & 0 & 0 & 0 & 0 & 0 & 0 & 0 & 0 & 0 & 0 & 0 & 0 \\
\hline 17 & 0 & 0 & 0 & 0 & 0 & 0 & 0 & 0 & 0 & .49 & 0 & 0 \\
\hline 18 & 0 & 0 & 0 & 0 & 0 & 0 & 0 & 0 & 0 & .42 & .06 & 0 \\
\hline 19 & 0 & 0 & 0 & 0 & 0 & 0 & 0 & 0 & 0 & 1.4 & .50 & 0 \\
\hline 20 & 0 & 0 & 0 & 0 & 0 & 0 & 0 & 0 & 0 & 4.2 & $0^{*}$ & 0 \\
\hline 21 & 0 & 0 & 0 & 0 & 0 & 0 & 0 & 0 & 0 & 1.3 & $0^{*}$ & 0 \\
\hline 22 & 0 & 0 & 0 & 0 & 0 & 0 & 0 & 0 & 0 & .09 & 0 & 0 \\
\hline 23 & 0 & 0 & 0 & 0 & 0 & 0 & 0 & 0 & 0 & .01 & 0 & 0 \\
\hline 24 & 0 & 0 & 0 & 0 & 0 & 0 & 0 & 0 & 0 & 0 & 0 & 0 \\
\hline 25 & 0 & 0 & 0 & 0 & 0 & 0 & 0 & 0 & 0 & 0 & 0 & 0 \\
\hline 26 & 0 & 0 & 0 & 0 & 0 & 0 & 0 & 0 & 0 & 0 & 0 & 0 \\
\hline 27 & 0 & 0 & 0 & 0 & 0 & 0 & 0 & 0 & 0 & 0 & 0 & 0 \\
\hline 28 & 0 & 0 & 0 & 0 & 0 & 0 & 0 & 0 & 0 & 0 & 0 & 0 \\
\hline 29 & 0 & 0 & 0 & 0 & ------ & 0 & 0 & 0 & 0 & .02 & 0 & 0 \\
\hline 30 & 0 & 0 & 0 & 0 & ----- & 0 & 0 & 0 & 0 & .01 & 0 & 0 \\
\hline 31 & 0 & ------ & 0 & 0 & ----- & 0 & ----- & 0 & ----- & 0 & 0 & ----- \\
\hline Total & 0 & 0 & 0 & 0 & 0 & 0 & 0 & 0 & 3.17 & 8.00 & 0.57 & 1.62 \\
\hline Mean & 0 & 0 & 0 & 0 & 0 & 0 & 0 & 0 & .11 & .26 & .018 & .054 \\
\hline Max & 0 & 0 & 0 & 0 & 0 & 0 & 0 & 0 & 1.9 & 4.2 & .50 & 1.3 \\
\hline Min & 0 & 0 & 0 & 0 & 0 & 0 & 0 & 0 & 0 & 0 & 0 & 0 \\
\hline Ac-Ft & 0 & 0 & .01 & 0 & 0 & 0 & 0 & 0 & 6.3 & 16 & 1.1 & 3.2 \\
\hline Wtr Year & 2000 & Total & 13.36 & & Mean & .037 & Max & 4.2 & Min & 0 & $\mathrm{Ac}-\mathrm{Ft}$ & 27 \\
\hline Cal Year & 1999 & Total & 44.96 & & Mean & .12 & Max & 3.5 & Min & 0 & $\mathrm{Ac}-\mathrm{Ft}$ & 89 \\
\hline
\end{tabular}

${ }^{*}$ Estimated. 


\section{E060 Pueblo Canyon near Los Alamos, NM}

Location. Lat $35^{\circ} 52^{\prime} 50^{\prime \prime}$, long $106^{\circ} 13^{\prime} 1 "$ ", in NE 1/4 NE 1/4 sec. 20, T. 19 N, R. 7 E, Santa Fe County on right bank at state highway maintenance yard $200 \mathrm{ft}$. north of NM State Highway 502, and 4.2. mi east of Los Alamos.

Drainage Area. $6.94 \mathrm{mi}^{2}$.

Period of Record. January 1992 to September 30, 2000.

Gage. Data logger with cellular telemetry. Elevation of gage is 6,356 ft above National Geodetic Vertical Datum of 1929, from GPS survey.

Remarks. Records fair. No diversion above station. Perennial flow is primarily from effluent.

Extremes for Period of Record. Maximum discharge $114 \mathrm{ft}^{3} / \mathrm{s}$, September 8, 2000, gage height $7.97 \mathrm{ft}$. No flow at times.

Extremes for Current Water Year. Maximum discharge $114 \mathrm{ft}^{3} / \mathrm{s}$ at $2010 \mathrm{hrs}$, September 8, gage height $7.97 \mathrm{ft}$. No flow at times.

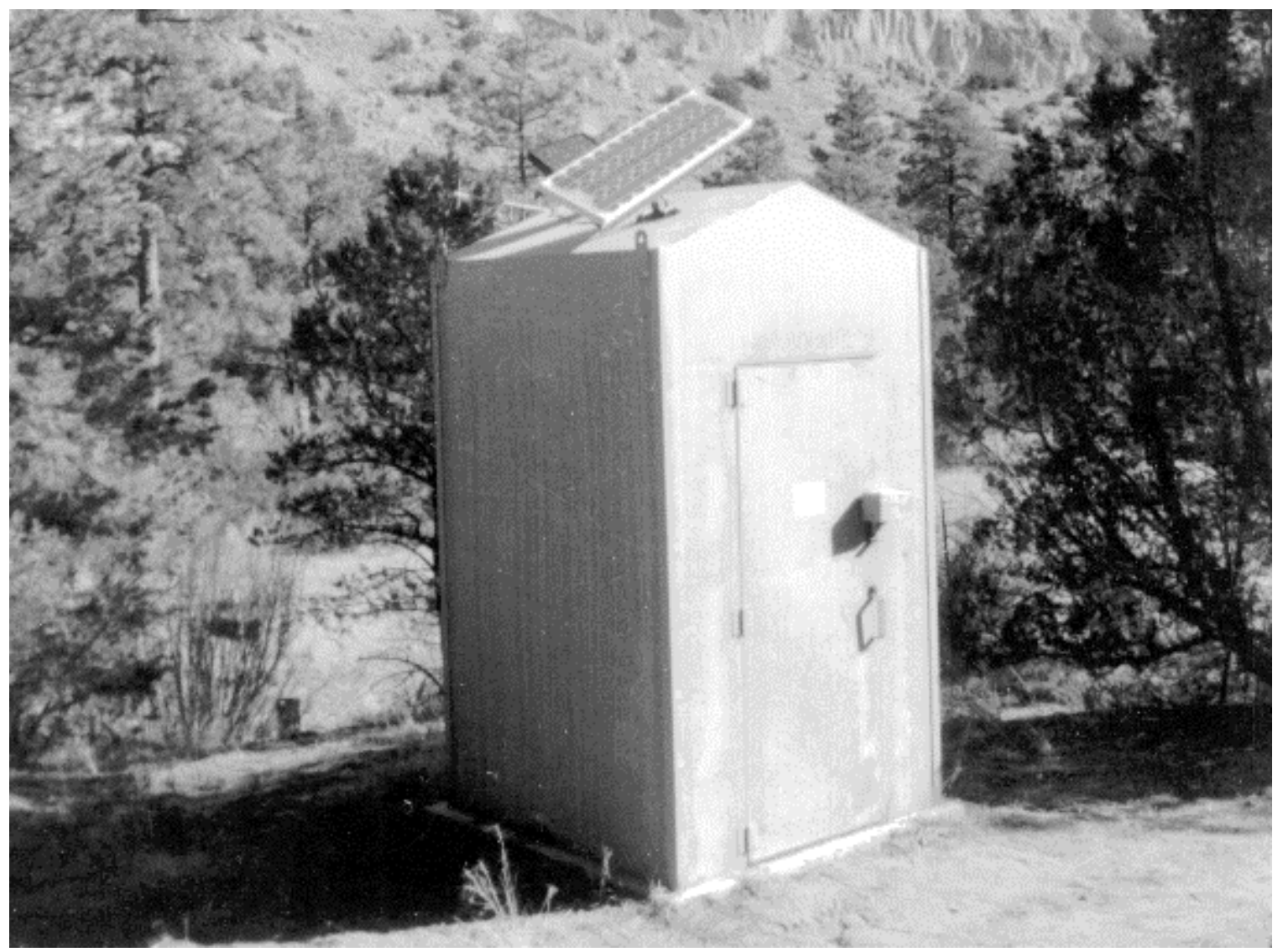




\section{E060 Pueblo Canyon near Los Alamos, NM}

Daily Mean Discharge in Cubic Feet Per Second

Water Year October 1999 to September 2000

\begin{tabular}{|c|c|c|c|c|c|c|c|c|c|c|c|c|}
\hline DAY & OCT & NOV & DEC & JAN & FEB & MAR & APR & MAY & JUN & JUL & AUG & SEP \\
\hline 1 & 1.2 & 2.7 & 2.2 & .70 & .55 & 2.4 & .55 & .20 & .07 & .20 & .13 & .01 \\
\hline 2 & .95 & 1.1 & 2.8 & .70 & .60 & 2.8 & .55 & .16 & .02 & .13 & .13 & .06 \\
\hline 3 & 1.0 & 2.0 & 3.1 & .65 & .60 & 3.0 & .50 & .15 & .19 & .13 & 4.0 & .02 \\
\hline 4 & 1.6 & 1.6 & 2.5 & .65 & .65 & 2.1 & .33 & .15 & .16 & .13 & .16 & .02 \\
\hline 5 & .96 & 1.9 & 2.3 & .65 & .65 & 2.0 & .32 & .15 & .21 & .13 & .28 & .11 \\
\hline 6 & 1.6 & 1.9 & 2.2 & .60 & .65 & 2.1 & .18 & .14 & .15 & .14 & .61 & .01 \\
\hline 7 & 1.3 & 1.5 & 2.2 & .55 & .70 & 2.3 & .20 & .14 & .13 & .12 & .24 & 0 \\
\hline 8 & 1.7 & 2.6 & 2.2 & .55 & .75 & 2.4 & .17 & .16 & .14 & .25 & .20 & 4.9 \\
\hline 9 & 1.4 & 1.8 & 2.2 & .50 & .80 & 2.0 & .21 & .20 & .14 & .15 & .16 & 1.5 \\
\hline 10 & 1.3 & 2.5 & 2.1 & .45 & .80 & 2.0 & .21 & .14 & .16 & .29 & .19 & .33 \\
\hline 11 & 1.8 & 1.7 & 2.1 & .45 & .85 & 1.6 & .23 & .13 & .18 & .30 & .09 & .37 \\
\hline 12 & 1.5 & 2.5 & 2.1 & .45 & .90 & 1.6 & .28 & .11 & .14 & .27 & 1.1 & .07 \\
\hline 13 & 1.7 & 1.7 & 2.0 & .50 & .90 & 1.4 & .27 & .13 & .13 & .23 & .21 & .09 \\
\hline 14 & 1.5 & 2.9 & 1.9 & .50 & .95 & 1.6 & .20 & .14 & .14 & .20 & .25 & .08 \\
\hline 15 & 1.8 & 2.9 & 1.8 & .55 & .95 & 1.6 & .17 & .13 & .14 & .17 & .10 & .05 \\
\hline 16 & 1.7 & 2.0 & 1.8 & .60 & .95 & 1.3 & .22 & .10 & .09 & .14 & .01 & .06 \\
\hline 17 & 2.7 & 2.7 & 1.7 & .65 & .95 & 1.6 & .19 & .04 & .05 & .12 & .01 & .08 \\
\hline 18 & 2.9 & 2.7 & 1.5 & .65 & 1.0 & .88 & .42 & .01 & .14 & .10 & .33 & .13 \\
\hline 19 & 2.7 & 2.0 & 1.4 & .70 & 1.0 & 1.2 & .25 & 0 & .13 & .09 & .82 & .46 \\
\hline 20 & 2.7 & 2.5 & 1.3 & .70 & 1.0 & 1.4 & .25 & 0 & .13 & .10 & .27 & .08 \\
\hline 21 & 2.4 & 2.9 & 1.0 & .70 & 1.2 & 1.2 & .17 & 0 & .14 & .10 & .35 & .05 \\
\hline 22 & 1.2 & 3.1 & 1.0 & .70 & 1.3 & 1.1 & .19 & 0 & .11 & .10 & .19 & .16 \\
\hline 23 & 1.4 & 2.9 & .95 & .70 & 1.4 & .90 & .22 & 0 & .13 & .10 & .09 & .11 \\
\hline 24 & 2.4 & 3.0 & .90 & .65 & 1.4 & .88 & .17 & 0 & .13 & .10 & .05 & .23 \\
\hline 25 & 2.6 & 3.2 & .90 & .60 & 1.4 & .76 & .16 & 0 & .13 & .10 & .01 & .23 \\
\hline 26 & 2.0 & 3.1 & .85 & .55 & 1.5 & .87 & .15 & 0 & .11 & .10 & 0 & .43 \\
\hline 27 & 1.5 & 2.9 & .85 & .55 & 1.5 & .77 & .15 & .12 & .13 & .11 & .02 & .07 \\
\hline 28 & 2.4 & 2.3 & .85 & .50 & 1.5 & .38 & .14 & .14 & .12 & .11 & .01 & .07 \\
\hline 29 & 2.7 & 1.8 & .80 & .55 & ----- & .58 & .16 & .07 & .16 & .11 & .02 & .10 \\
\hline 30 & 2.3 & 2.1 & .75 & .55 & ----- & .59 & .16 & .10 & .36 & .11 & .01 & .33 \\
\hline 31 & 2.7 & ----- & .70 & .55 & ------ & .51 & $-\cdot---$ & .14 & - & .12 & .02 & ---- \\
\hline Total & 57.61 & 70.5 & 50.95 & 18.35 & 28.80 & 45.82 & 7.37 & 2.95 & 4.16 & 4.55 & 10.06 & 10.21 \\
\hline Mean & 1.86 & 2.35 & 1.64 & .59 & .99 & 1.48 & .25 & .095 & .14 & .15 & .32 & .34 \\
\hline Max & 2.9 & 3.2 & 3.1 & .70 & 1.5 & 3.0 & .55 & .20 & .36 & .30 & 4.0 & 4.9 \\
\hline Min & .95 & 1.1 & .70 & .45 & .55 & .38 & .14 & 0 & .02 & .09 & 0 & 0 \\
\hline$A c-F t$ & 114 & 140 & 102 & 37 & 58 & 91 & 15 & 6.0 & 8.5 & 9.1 & 20 & 20 \\
\hline Wtr Year & 2000 & Tota & 311 & & Mean & .85 & Max & 4.9 & Min & 0 & $\mathrm{Ac}-\mathrm{Ft}$ & 618 \\
\hline Cal Year & 1999 & Tota & 563 & & Mean & 1.54 & Max & 4.2 & Min & .40 & $\mathrm{Ac}-\mathrm{Ft}$ & 1120 \\
\hline
\end{tabular}




\section{E125 Sandia Canyon above Highway 4 near White Rock, NM}

Location. Lat 3551'32", long $106^{\circ} 13^{\prime} 34^{\prime \prime}$, SE 1/4 SW 1/4 sec. 20, T. 19 N, R.7 E, Santa Fe County, $0.25 \mathrm{mi}$ N of East Jemez Road and $0.5 \mathrm{mi}$ upstream from NM State Highway 4.

Drainage Area. $2.52 \mathrm{mi}^{2}$.

Period of Record. October 1993 to September 30, 2000.

Gage. Data logger with cellular telemetry and concrete control. Elevation of gage is 6,498 $\mathrm{ft}$. above National Geodetic Vertical Datum of 1929, from GPS survey.

Remarks. Water discharge records fair.

Extremes for Period of Record. Maximum discharge $13 \mathrm{ft}^{3} / \mathrm{s}$, September 8, 1995, gage height $1.82 \mathrm{ft}$. No flow most of time.

Extremes for Current Water Year. No flow all year.

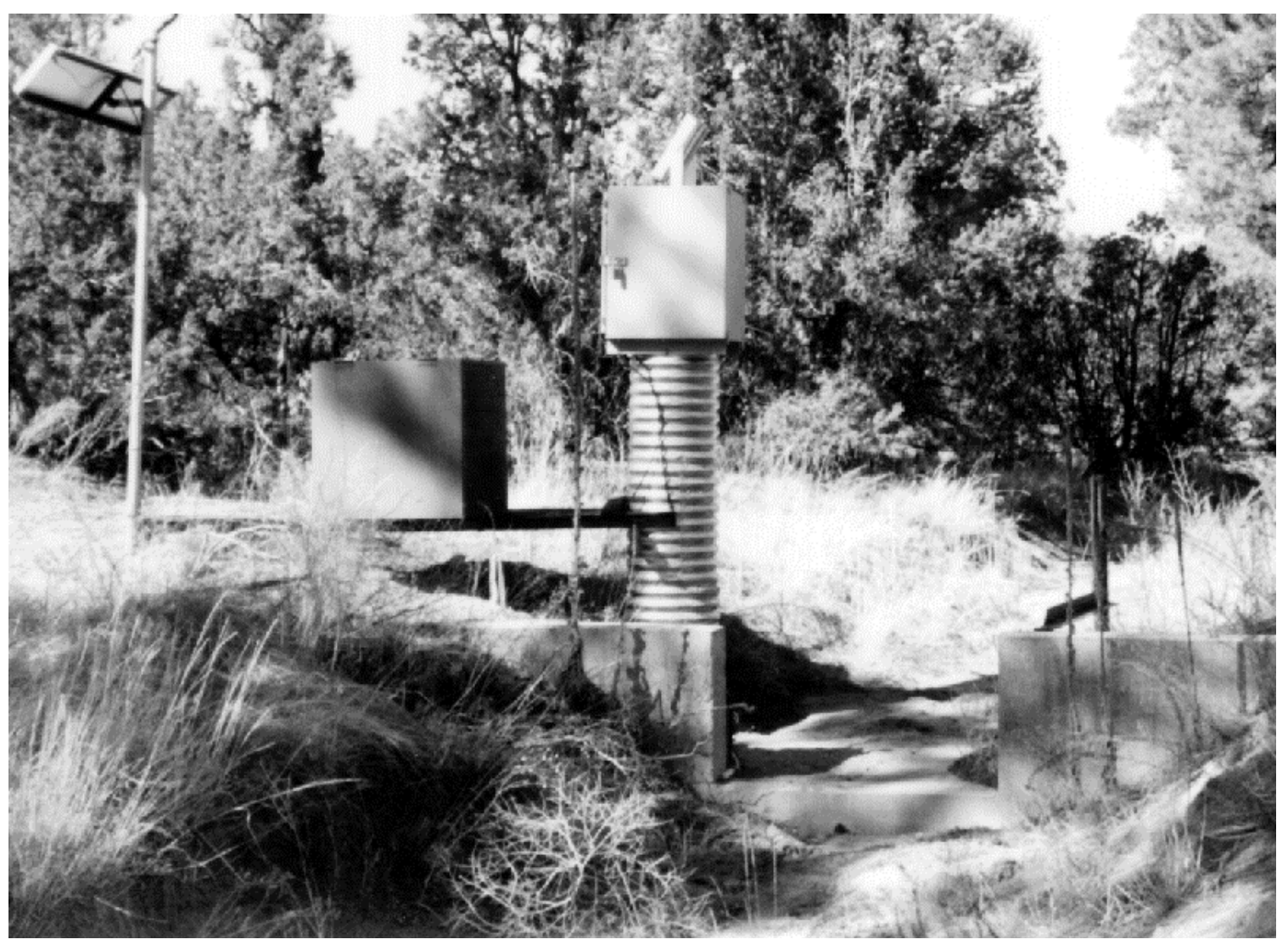




\section{E125 Sandia Canyon above Highway 4 near White Rock, NM}

Daily Mean Discharge in Cubic Feet per Second

Water Year October 1999 to September 2000

\begin{tabular}{|c|c|c|c|c|c|c|c|c|c|c|c|c|}
\hline DAY & OCT & NOV & DEC & JAN & FEB & MAR & APR & MAY & JUN & JUL & AUG & SEP \\
\hline 1 & 0 & 0 & 0 & 0 & 0 & 0 & 0 & 0 & 0 & 0 & 0 & 0 \\
\hline 2 & 0 & 0 & 0 & 0 & 0 & 0 & 0 & 0 & 0 & 0 & 0 & 0 \\
\hline 3 & 0 & 0 & 0 & 0 & 0 & 0 & 0 & 0 & 0 & 0 & 0 & 0 \\
\hline 4 & 0 & 0 & 0 & 0 & 0 & 0 & 0 & 0 & 0 & 0 & 0 & 0 \\
\hline 5 & 0 & 0 & 0 & 0 & 0 & 0 & 0 & 0 & 0 & 0 & 0 & 0 \\
\hline 6 & 0 & 0 & 0 & 0 & 0 & 0 & 0 & 0 & 0 & 0 & 0 & 0 \\
\hline 7 & 0 & 0 & 0 & 0 & 0 & 0 & 0 & 0 & 0 & 0 & 0 & 0 \\
\hline 8 & 0 & 0 & 0 & 0 & 0 & 0 & 0 & 0 & 0 & 0 & 0 & 0 \\
\hline 9 & 0 & 0 & 0 & 0 & 0 & 0 & 0 & 0 & 0 & 0 & 0 & 0 \\
\hline 10 & 0 & 0 & 0 & 0 & 0 & 0 & 0 & 0 & 0 & 0 & 0 & 0 \\
\hline 11 & 0 & 0 & 0 & 0 & 0 & 0 & 0 & 0 & 0 & 0 & 0 & 0 \\
\hline 12 & 0 & 0 & 0 & 0 & 0 & 0 & 0 & 0 & 0 & 0 & 0 & 0 \\
\hline 13 & 0 & 0 & 0 & 0 & 0 & 0 & 0 & 0 & 0 & 0 & 0 & 0 \\
\hline 14 & 0 & 0 & 0 & 0 & 0 & 0 & 0 & 0 & 0 & 0 & 0 & 0 \\
\hline 15 & 0 & 0 & 0 & 0 & 0 & 0 & 0 & 0 & 0 & 0 & 0 & 0 \\
\hline 16 & 0 & 0 & 0 & 0 & 0 & 0 & 0 & 0 & 0 & 0 & 0 & 0 \\
\hline 17 & 0 & 0 & 0 & 0 & 0 & 0 & 0 & 0 & 0 & 0 & 0 & 0 \\
\hline 18 & 0 & 0 & 0 & 0 & 0 & 0 & 0 & 0 & 0 & 0 & 0 & 0 \\
\hline 19 & 0 & 0 & 0 & 0 & 0 & 0 & 0 & 0 & 0 & 0 & 0 & 0 \\
\hline 20 & 0 & 0 & 0 & 0 & 0 & 0 & 0 & 0 & 0 & 0 & 0 & 0 \\
\hline 21 & 0 & 0 & 0 & 0 & 0 & 0 & 0 & 0 & 0 & 0 & 0 & 0 \\
\hline 22 & 0 & 0 & 0 & 0 & 0 & 0 & 0 & 0 & 0 & 0 & 0 & 0 \\
\hline 23 & 0 & 0 & 0 & 0 & 0 & 0 & 0 & 0 & 0 & 0 & 0 & 0 \\
\hline 24 & 0 & 0 & 0 & 0 & 0 & 0 & 0 & 0 & 0 & 0 & 0 & 0 \\
\hline 25 & 0 & 0 & 0 & 0 & 0 & 0 & 0 & 0 & 0 & 0 & 0 & 0 \\
\hline 26 & 0 & 0 & 0 & 0 & 0 & 0 & 0 & 0 & 0 & 0 & 0 & 0 \\
\hline 27 & 0 & 0 & 0 & 0 & 0 & 0 & 0 & 0 & 0 & 0 & 0 & 0 \\
\hline 28 & 0 & 0 & 0 & 0 & 0 & 0 & 0 & 0 & 0 & 0 & 0 & 0 \\
\hline 29 & 0 & 0 & 0 & 0 & ------ & 0 & 0 & 0 & 0 & 0 & 0 & 0 \\
\hline 30 & 0 & 0 & 0 & 0 & ------ & 0 & 0 & 0 & 0 & 0 & 0 & 0 \\
\hline 31 & 0 & ------ & 0 & 0 & -.--- & 0 & ------ & 0 & ----- & 0 & 0 & ---.-- \\
\hline Total & 0 & 0 & 0 & 0 & 0 & 0 & 0 & 0 & 0 & 0 & 0 & 0 \\
\hline Mean & 0 & 0 & 0 & 0 & 0 & 0 & 0 & 0 & 0 & 0 & 0 & 0 \\
\hline Max & 0 & 0 & 0 & 0 & 0 & 0 & 0 & 0 & 0 & 0 & 0 & 0 \\
\hline Min & 0 & 0 & 0 & 0 & 0 & 0 & 0 & 0 & 0 & 0 & 0 & 0 \\
\hline Ac-Ft & 0 & 0 & 0 & 0 & 0 & 0 & 0 & 0 & 0 & 0 & 0 & 0 \\
\hline Wtr Year & 2000 & Total & 0 & & lean & & $\operatorname{Max}$ & 0 & Min & 0 & Ac-Ft & 0 \\
\hline Cal Year & 1999 & Total & 0 & & lean & & Max & 0 & Min & 0 & Ac-Ft & 0 \\
\hline
\end{tabular}


Location. Lat 3551'55", long 106²1'46", SW 1/4 NE 1/2 sec. 22, T. 19 N, R. 6 E, Los Alamos County, $0.6 \mathrm{mi}$ of Pajarito Road and $0.25 \mathrm{mi} \mathrm{N}$ of LANL TA-50 and 1/4 mi below TA-50 outfall.

Drainage Area. $0.49 \mathrm{mi}^{2}$.

Period of Record. May 10, 1995, to September 30, 2000.

Gage. Data logger with cellular telemetry and steel "fabricated" Parshall Flume as low-water control. Elevation of gage is $7062.50 \mathrm{ft}$ above National Geodetic Vertical Datum of 1929, from survey.

Remarks. Water discharge records poor. Flow is mostly effluent from LANL TA-50, liquid radiological waste plant.

Extremes Outside Period of Record. Flow of $34 \mathrm{ft}^{3} / \mathrm{s}$ occurred August 19, 1970, gage height $3.07 \mathrm{ft}$, from old data files of USGS.

Extremes for Period of Record. Maximum discharge $38 \mathrm{ft}^{3} / \mathrm{s}$, August 17, 1997, gage height $3.19 \mathrm{ft}$. No flow at times.

Extremes for Current Water Year. Maximum discharge $12 \mathrm{ft}^{3} / \mathrm{s}$ at $1345 \mathrm{hrs}$, August 19, gage height $2.11 \mathrm{ft}$. No flow at times.

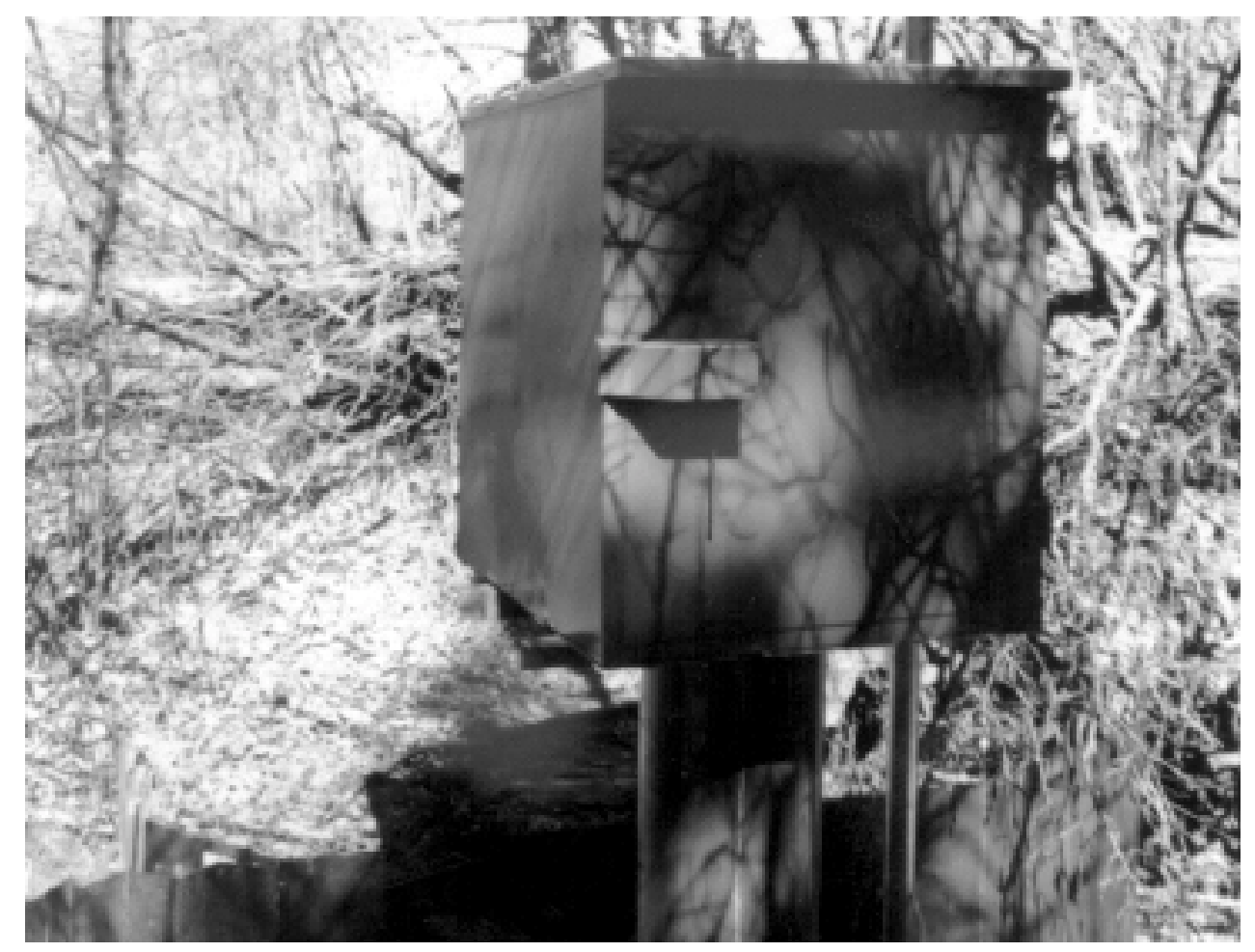




\section{E200 Mortandad Canyon at TA-50 near Los Alamos, NM}

Daily Mean Discharge in Cubic Feet per Second

Water Year October 1999 to September 2000

\begin{tabular}{|c|c|c|c|c|c|c|c|c|c|c|c|c|}
\hline DAY & OCT & NOV & DEC & JAN & FEB & MAR & APR & MAY & JUN & JUL & AUG & SEP \\
\hline 1 & .02 & .03 & 0 & 0 & .06 & .03 & 0 & .03 & .05 & 0 & .02 & .02 \\
\hline 2 & 0 & .05 & .03 & .03 & .03 & .06 & 0 & .03 & .08 & 0 & .02 & 0 \\
\hline 3 & 0 & .04 & .03 & .03 & .03 & .05 & .06 & .03 & .01 & .03 & .02 & 0 \\
\hline 4 & .06 & 0 & 0 & .03 & .03 & 0 & .06 & .03 & 0 & .01 & .02 & 0 \\
\hline 5 & .07 & 0 & 0 & .01 & 0 & 0 & 0 & .03 & .02 & .03 & 0 & .01 \\
\hline 6 & .03 & 0 & .05 & .06 & .03 & .03 & 0 & 0 & .03 & .03 & 0 & .01 \\
\hline 7 & .03 & 0 & .03 & 0 & .03 & .03 & .06 & 0 & 0 & .03 & .02 & .02 \\
\hline 8 & 0 & 0 & .03 & .01 & .03 & .03 & 0 & 0 & 0 & 0 & .04 & .01 \\
\hline 9 & 0 & 0 & .04 & .01 & .03 & .03 & 0 & 0 & .03 & 0 & .01 & 0 \\
\hline 10 & .03 & .03 & 0 & .03 & .03 & .06 & .06 & .03 & 0 & .03 & .04 & 0 \\
\hline 11 & 0 & .02 & 0 & .04 & .03 & 0 & .03 & .01 & 0 & .03 & 0 & .01 \\
\hline 12 & .03 & .02 & 0 & .03 & 0 & 0 & .03 & 0 & .03 & .03 & 0 & .01 \\
\hline 13 & .03 & .03 & .01 & .02 & 0 & .03 & .06 & 0 & .03 & .06 & 0 & 0 \\
\hline 14 & .04 & .03 & .03 & .02 & .06 & .03 & .03 & 0 & .03 & .01 & 0 & .01 \\
\hline 15 & .04 & .06 & .01 & 0 & .03 & .03 & .01 & .06 & .06 & 0 & .01 & .01 \\
\hline 16 & .02 & .09 & .03 & .02 & .06 & .08 & 0 & 0 & .06 & 0 & .01 & 0 \\
\hline 17 & .02 & .10 & .03 & 0 & .03 & .03 & .06 & 0 & 0 & .03 & .05 & 0 \\
\hline 18 & .06 & .03 & 0 & .02 & .03 & 0 & .03 & .03 & .10 & .03 & .16 & .01 \\
\hline 19 & .04 & .05 & .01 & .02 & 0 & 0 & .03 & .03 & .03 & .06 & .48 & 0 \\
\hline 20 & .04 & .02 & .06 & .02 & .03 & .03 & 0 & 0 & .01 & .06 & .02 & .01 \\
\hline 21 & .07 & .02 & 0 & .02 & 0 & .03 & 0 & 0 & 0 & .03 & .06 & .02 \\
\hline 22 & .03 & .04 & .04 & 0 & .06 & .14 & 0 & 0 & 0 & 0 & .02 & .02 \\
\hline 23 & 0 & .03 & .03 & 0 & .03 & .07 & 0 & .03 & .03 & 0 & .04 & 0 \\
\hline 24 & 0 & .08 & .01 & .02 & .03 & .03 & .03 & .03 & 0 & .03 & .02 & 0 \\
\hline 25 & .04 & .01 & .01 & .02 & .03 & 0 & .03 & .03 & 0 & .04 & .02 & .02 \\
\hline 26 & .02 & .01 & .03 & .03 & 0 & 0 & .03 & .03 & 0 & .05 & 0 & 0 \\
\hline 27 & .04 & .02 & 0 & .02 & 0 & .03 & .03 & 0 & .03 & .02 & 0 & 0 \\
\hline 28 & .03 & .05 & .02 & .02 & .03 & .06 & .03 & .03 & .03 & .02 & .02 & .04 \\
\hline 29 & .04 & 0 & 0 & .01 & ----- & .06 & .01 & .01 & .06 & .09 & .02 & 0 \\
\hline 30 & 0 & .02 & .01 & .02 & ----- & .03 & 0 & 0 & .11 & .01 & .02 & 0 \\
\hline 31 & 0 & 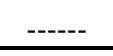 & .03 & .01 & ----- & 0 & $-\cdots--$ & .05 & ----- & .05 & 0 & $-\cdots$ \\
\hline Total & 0.83 & 0.88 & 0.57 & 0.57 & 0.78 & 1.00 & 0.68 & 0.52 & 0.83 & 0.81 & 1.14 & 0.23 \\
\hline Mean & .027 & .029 & .018 & .018 & .027 & .032 & .023 & .017 & .028 & .026 & .037 & .008 \\
\hline Max & .07 & .10 & .06 & .06 & .06 & .14 & .06 & .06 & .11 & .09 & .48 & .04 \\
\hline Min & 0 & 0 & 0 & 0 & 0 & 0 & 0 & 0 & 0 & 0 & 0 & 0 \\
\hline $\mathrm{Ac}-\mathrm{Ft}$ & 1.5 & 1.7 & 1.1 & 1.2 & 1.5 & 2.0 & 1.3 & 1.0 & 1.6 & 1.6 & 2.3 & .5 \\
\hline Wtr Year & 2000 & Tota & & 84 & Mean & .024 & Max & .48 & Min & 0 & $\mathrm{Ac}-\mathrm{Ft}$ & 17 \\
\hline Cal Year & 1999 & Tot & & & Mean & .037 & Max & .91 & Min & 0 & $\mathrm{Ac}-\mathrm{Ft}$ & 26 \\
\hline
\end{tabular}




\section{E202 Mortandad Canyon at Entrance to Sediment Traps}

Location. Lat 3551'39", long 106¹6'15", NE 1/4 SW 1/4 sec. 23, T. 19 N, R. 6 E, Los Alamos County, 4.3 mi upstream from NM State Highway 4.

Drainage Area. $0.81 \mathrm{mi}^{2}$.

Period of Record. October 1, 1997, to September 30, 2000.

Gage. Data logger with cellular telemetry and $2 \mathrm{ft}$ Parshall Flume. Elevation of gage is $6833.06 \mathrm{ft}$ above National Geodetic Vertical Datum of 1929.

Extremes for Period of Record. Maxium discharge $6.4 \mathrm{ft}^{3} / \mathrm{s}$, gage height $0.87 \mathrm{ft}$, August 17, 1997. No flow most of time.

Extremes for Current Water Year. Maxium discharge $1.6 \mathrm{ft}^{3} / \mathrm{s}$ at $2000 \mathrm{hrs,} \mathrm{July} \mathrm{29,} \mathrm{gage} \mathrm{height}$ $0.38 \mathrm{ft}$. No flow most of time.

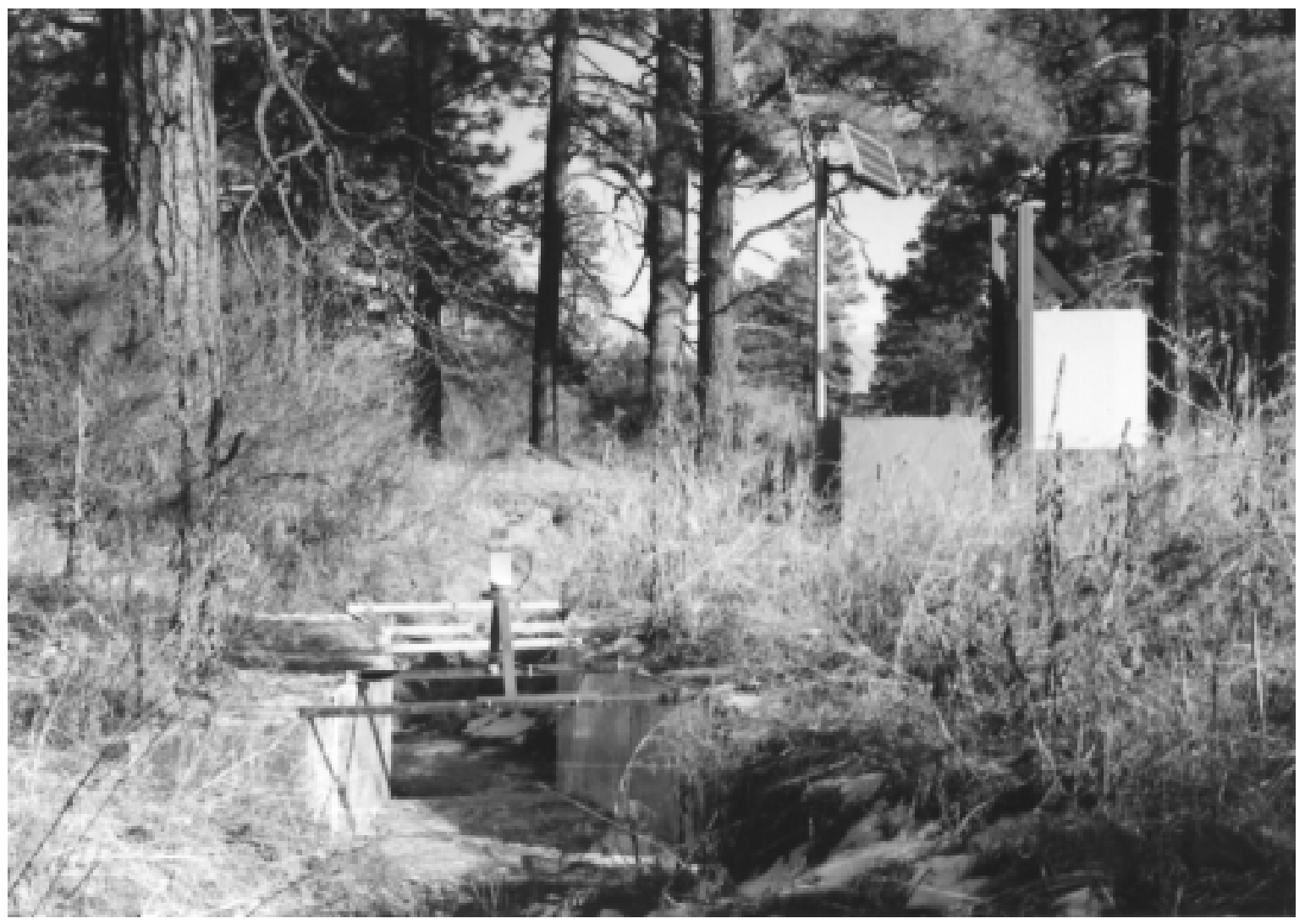




\section{E202 Mortandad Canyon at Entrance to Sediment Traps}

Daily Mean Discharge in Cubic Feet per Second

Water Year October 1999 to September 2000

\begin{tabular}{|c|c|c|c|c|c|c|c|c|c|c|c|c|}
\hline DAY & ОСТ & NOV & DEC & JAN & FEB & MAR & APR & MAY & JUN & JUL & AUG & SEP \\
\hline 1 & 0 & 0 & 0 & 0 & 0 & 0 & 0 & 0 & 0 & 0 & 0 & 0 \\
\hline 2 & 0 & 0 & 0 & 0 & 0 & 0 & 0 & 0 & 0 & 0 & 0 & 0 \\
\hline 3 & 0 & 0 & 0 & 0 & 0 & 0 & 0 & 0 & 0 & 0 & 0 & 0 \\
\hline 4 & 0 & 0 & 0 & 0 & 0 & 0 & 0 & 0 & 0 & 0 & 0 & 0 \\
\hline 5 & 0 & 0 & 0 & 0 & 0 & 0 & 0 & 0 & 0 & 0 & 0 & 0 \\
\hline 6 & 0 & 0 & 0 & 0 & 0 & 0 & 0 & 0 & 0 & 0 & 0 & 0 \\
\hline 7 & 0 & 0 & 0 & 0 & 0 & 0 & 0 & 0 & 0 & 0 & 0 & 0 \\
\hline 8 & 0 & 0 & 0 & 0 & 0 & 0 & 0 & 0 & 0 & 0 & 0 & 0 \\
\hline 9 & 0 & 0 & 0 & 0 & 0 & 0 & 0 & 0 & 0 & 0 & 0 & 0 \\
\hline 10 & 0 & 0 & 0 & 0 & 0 & 0 & 0 & 0 & 0 & 0 & 0 & 0 \\
\hline 11 & 0 & 0 & 0 & 0 & 0 & 0 & 0 & 0 & 0 & 0 & 0 & 0 \\
\hline 12 & 0 & 0 & 0 & 0 & 0 & 0 & 0 & 0 & 0 & 0 & 0 & 0 \\
\hline 13 & 0 & 0 & 0 & 0 & 0 & 0 & 0 & 0 & 0 & 0 & 0 & 0 \\
\hline 14 & 0 & 0 & 0 & 0 & 0 & 0 & 0 & 0 & 0 & 0 & 0 & 0 \\
\hline 15 & 0 & 0 & 0 & 0 & 0 & 0 & 0 & 0 & 0 & 0 & 0 & 0 \\
\hline 16 & 0 & 0 & 0 & 0 & 0 & 0 & 0 & 0 & 0 & 0 & 0 & 0 \\
\hline 17 & 0 & 0 & 0 & 0 & 0 & 0 & 0 & 0 & 0 & 0 & 0 & 0 \\
\hline 18 & 0 & 0 & 0 & 0 & 0 & 0 & 0 & 0 & 0 & 0 & 0 & 0 \\
\hline 19 & 0 & 0 & 0 & 0 & 0 & 0 & 0 & 0 & 0 & 0 & .08 & 0 \\
\hline 20 & 0 & 0 & 0 & 0 & 0 & 0 & 0 & 0 & 0 & 0 & 0 & 0 \\
\hline 21 & 0 & 0 & 0 & 0 & 0 & 0 & 0 & 0 & 0 & 0 & 0 & 0 \\
\hline 22 & 0 & 0 & 0 & 0 & 0 & 0 & 0 & 0 & 0 & 0 & 0 & 0 \\
\hline 23 & 0 & 0 & 0 & 0 & 0 & 0 & 0 & 0 & 0 & 0 & 0 & 0 \\
\hline 24 & 0 & 0 & 0 & 0 & 0 & 0 & 0 & 0 & 0 & 0 & 0 & 0 \\
\hline 25 & 0 & 0 & 0 & 0 & 0 & 0 & 0 & 0 & 0 & 0 & 0 & 0 \\
\hline 26 & 0 & 0 & 0 & 0 & 0 & 0 & 0 & 0 & 0 & 0 & 0 & 0 \\
\hline 27 & 0 & 0 & 0 & 0 & 0 & 0 & 0 & 0 & 0 & 0 & 0 & 0 \\
\hline 28 & 0 & 0 & 0 & 0 & 0 & 0 & 0 & 0 & 0 & 0 & 0 & 0 \\
\hline 29 & 0 & 0 & 0 & 0 & ----- & 0 & 0 & 0 & 0 & .08 & 0 & 0 \\
\hline 30 & 0 & 0 & 0 & 0 & ----- & 0 & 0 & 0 & 0 & .01 & 0 & 0 \\
\hline 31 & 0 & ----- & 0 & 0 & ----- & 0 & ----- & 0 & ----- & 0 & 0 & ----- \\
\hline Total & 0 & 0 & 0 & 0 & 0 & 0 & 0 & 0 & 0 & 0.09 & .08 & 0 \\
\hline Mean & 0 & 0 & 0 & 0 & 0 & 0 & 0 & 0 & 0 & .003 & .003 & 0 \\
\hline Max & 0 & 0 & 0 & 0 & 0 & 0 & 0 & 0 & 0 & .08 & .08 & 0 \\
\hline Min & 0 & 0 & 0 & 0 & 0 & 0 & 0 & 0 & 0 & 0 & 0 & 0 \\
\hline Ac-Ft & 0 & 0 & 0 & 0 & 0 & 0 & 0 & 0 & 0 & .2 & .2 & 0 \\
\hline Wtr Year & 2000 & Total & & & Mean & 0 & Max & .08 & Min & 0 & $\mathrm{Ac}-\mathrm{Ft}$ & .4 \\
\hline Cal Year & 1999 & Total & c & & Mean & 0 & Max & 0 & Min & 0 & $\mathrm{Ac}-\mathrm{Ft}$ & 0 \\
\hline
\end{tabular}




\section{E203 Mortandad Canyon below Sediment Traps}

Location. Lat 3551'39", long 106¹6'6", NE 1/4 SW 1/4 sec. 23, T. 19 N, R. 6 E, Los Alamos County, at exit from sediment collection traps, 4.2 mi upstream from NM State Highway 4.

Drainage Area. $0.9 \mathrm{mi}^{2}$, approximately.

Period of Record. October 1, 1996, to September 30, 2000.

Gage. Data logger and 6-in. Parshall flume. Elevation of gage is 6,811.52 ft above National Geodetic Vertical Datum of 1929.

Extremes for Period of Record. No flow for period.

Extremes for Current Water Year. No flow all year.

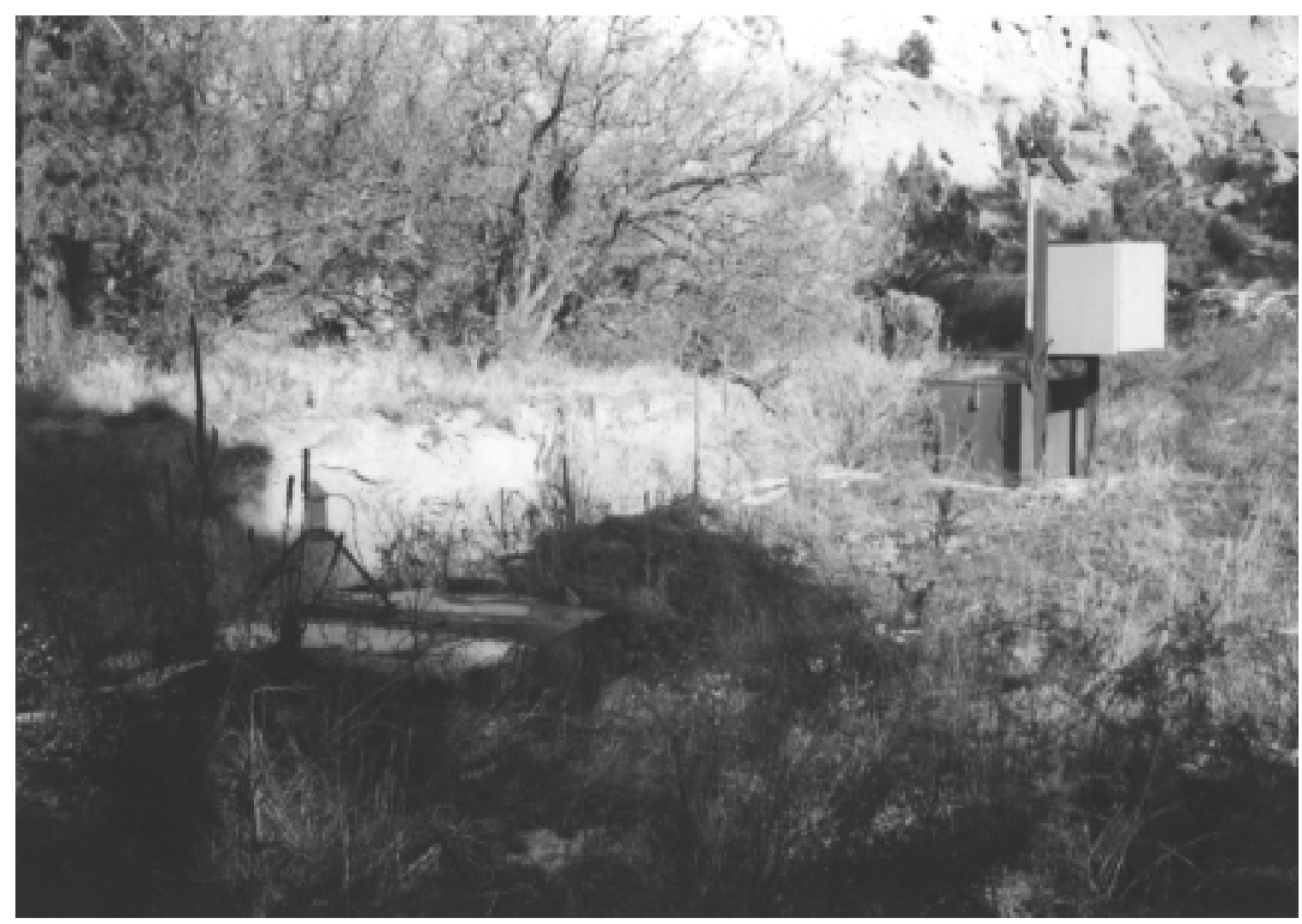




\section{E203 Mortandad Canyon below Sediment Traps}

Daily Mean Discharge in Cubic Feet per Second

Water Year October 1999 to September 2000

\begin{tabular}{|c|c|c|c|c|c|c|c|c|c|c|c|c|}
\hline DAY & OCT & NOV & DEC & JAN & FEB & MAR & APR & MAY & JUN & JUL & AUG & SEP \\
\hline 1 & 0 & 0 & 0 & 0 & 0 & 0 & 0 & 0 & 0 & 0 & 0 & 0 \\
\hline 2 & 0 & 0 & 0 & 0 & 0 & 0 & 0 & 0 & 0 & 0 & 0 & 0 \\
\hline 3 & 0 & 0 & 0 & 0 & 0 & 0 & 0 & 0 & 0 & 0 & 0 & 0 \\
\hline 4 & 0 & 0 & 0 & 0 & 0 & 0 & 0 & 0 & 0 & 0 & 0 & 0 \\
\hline 5 & 0 & 0 & 0 & 0 & 0 & 0 & 0 & 0 & 0 & 0 & 0 & 0 \\
\hline 6 & 0 & 0 & 0 & 0 & 0 & 0 & 0 & 0 & 0 & 0 & 0 & 0 \\
\hline 7 & 0 & 0 & 0 & 0 & 0 & 0 & 0 & 0 & 0 & 0 & 0 & 0 \\
\hline 8 & 0 & 0 & 0 & 0 & 0 & 0 & 0 & 0 & 0 & 0 & 0 & 0 \\
\hline 9 & 0 & 0 & 0 & 0 & 0 & 0 & 0 & 0 & 0 & 0 & 0 & 0 \\
\hline 10 & 0 & 0 & 0 & 0 & 0 & 0 & 0 & 0 & 0 & 0 & 0 & 0 \\
\hline 11 & 0 & 0 & 0 & 0 & 0 & 0 & 0 & 0 & 0 & 0 & 0 & 0 \\
\hline 12 & 0 & 0 & 0 & 0 & 0 & 0 & 0 & 0 & 0 & 0 & 0 & 0 \\
\hline 13 & 0 & 0 & 0 & 0 & 0 & 0 & 0 & 0 & 0 & 0 & 0 & 0 \\
\hline 14 & 0 & 0 & 0 & 0 & 0 & 0 & 0 & 0 & 0 & 0 & 0 & 0 \\
\hline 15 & 0 & 0 & 0 & 0 & 0 & 0 & 0 & 0 & 0 & 0 & 0 & 0 \\
\hline 16 & 0 & 0 & 0 & 0 & 0 & 0 & 0 & 0 & 0 & 0 & 0 & 0 \\
\hline 17 & 0 & 0 & 0 & 0 & 0 & 0 & 0 & 0 & 0 & 0 & 0 & 0 \\
\hline 18 & 0 & 0 & 0 & 0 & 0 & 0 & 0 & 0 & 0 & 0 & 0 & 0 \\
\hline 19 & 0 & 0 & 0 & 0 & 0 & 0 & 0 & 0 & 0 & 0 & 0 & 0 \\
\hline 20 & 0 & 0 & 0 & 0 & 0 & 0 & 0 & 0 & 0 & 0 & 0 & 0 \\
\hline 21 & 0 & 0 & 0 & 0 & 0 & 0 & 0 & 0 & 0 & 0 & 0 & 0 \\
\hline 22 & 0 & 0 & 0 & 0 & 0 & 0 & 0 & 0 & 0 & 0 & 0 & 0 \\
\hline 23 & 0 & 0 & 0 & 0 & 0 & 0 & 0 & 0 & 0 & 0 & 0 & 0 \\
\hline 24 & 0 & 0 & 0 & 0 & 0 & 0 & 0 & 0 & 0 & 0 & 0 & 0 \\
\hline 25 & 0 & 0 & 0 & 0 & 0 & 0 & 0 & 0 & 0 & 0 & 0 & 0 \\
\hline 26 & 0 & 0 & 0 & 0 & 0 & 0 & 0 & 0 & 0 & 0 & 0 & 0 \\
\hline 27 & 0 & 0 & 0 & 0 & 0 & 0 & 0 & 0 & 0 & 0 & 0 & 0 \\
\hline 28 & 0 & 0 & 0 & 0 & 0 & 0 & 0 & 0 & 0 & 0 & 0 & 0 \\
\hline 29 & 0 & 0 & 0 & 0 & ----- & 0 & 0 & 0 & 0 & 0 & 0 & 0 \\
\hline 30 & 0 & 0 & 0 & 0 & ----- & 0 & 0 & 0 & 0 & 0 & 0 & 0 \\
\hline 31 & 0 & ----- & 0 & 0 & ----- & 0 & ----- & 0 & ----- & 0 & 0 & -..- \\
\hline Total & 0 & 0 & 0 & 0 & 0 & 0 & 0 & 0 & 0 & 0 & 0 & 0 \\
\hline Mean & 0 & 0 & 0 & 0 & 0 & 0 & 0 & 0 & 0 & 0 & 0 & 0 \\
\hline Max & 0 & 0 & 0 & 0 & 0 & 0 & 0 & 0 & 0 & 0 & 0 & 0 \\
\hline Min & 0 & 0 & 0 & 0 & 0 & 0 & 0 & 0 & 0 & 0 & 0 & 0 \\
\hline $\mathrm{Ac}-\mathrm{Ft}$ & 0 & 0 & 0 & 0 & 0 & 0 & 0 & 0 & 0 & 0 & 0 & 0 \\
\hline Wtr Year & 2000 & Total & 0 & & Mean & 0 & Max & 0 & Min & 0 & $\mathrm{Ac}-\mathrm{Ft}$ & 0 \\
\hline Cal Year & 1999 & Total & 0 & & Mean & 0 & Max & 0 & Min & 0 & $A c-F t$ & 0 \\
\hline
\end{tabular}




\section{E204 Mortandad Canyon at Laboratory Boundary}

Location. Lat 35 51'21", long 106²14'43", NW 1/4 NW 1/4 sec. 30, T. 19, R. 7 E, Santa Fe County, $100 \mathrm{ft}$ upstream from LANL/San Ildefonso Indian Reservation Boundary and $2.8 \mathrm{mi}$ upstream from NM State Highway 4.

Drainage Area. $1.67 \mathrm{mi}^{2}$.

Period of Record. October 1, 1993, to September 30, 2000.

Gage. Data logger with cellular telemetry and concrete control. Elevation of gage is 6,657.66 ft above National Geodetic Vertical Datum of 1929, from survey.

Extremes for Period of Record. No flow for period.

Extremes for Current Water Year. No flow for year.

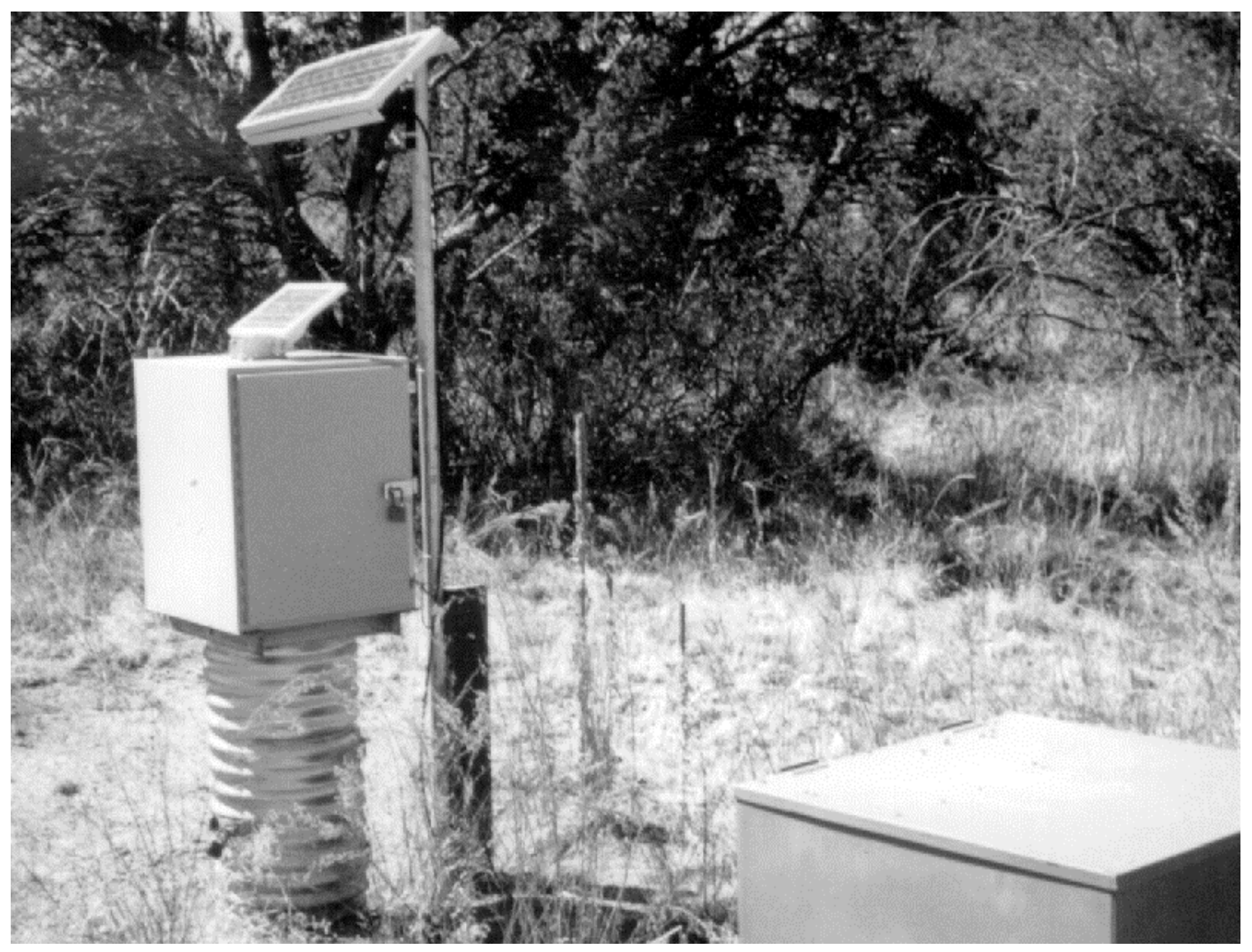




\section{E204 Mortandad Canyon at Laboratory Boundary}

Daily Mean Discharge in Cubic Feet per Second

Water Year October 1999 to September 2000

\begin{tabular}{|c|c|c|c|c|c|c|c|c|c|c|c|c|}
\hline DAY & OCT & NOV & DEC & JAN & FEB & MAR & APR & MAY & JUN & JUL & AUG & SEP \\
\hline 1 & 0 & 0 & 0 & 0 & 0 & 0 & 0 & 0 & 0 & 0 & 0 & 0 \\
\hline 2 & 0 & 0 & 0 & 0 & 0 & 0 & 0 & 0 & 0 & 0 & 0 & 0 \\
\hline 3 & 0 & 0 & 0 & 0 & 0 & 0 & 0 & 0 & 0 & 0 & 0 & 0 \\
\hline 4 & 0 & 0 & 0 & 0 & 0 & 0 & 0 & 0 & 0 & 0 & 0 & 0 \\
\hline 5 & 0 & 0 & 0 & 0 & 0 & 0 & 0 & 0 & 0 & 0 & 0 & 0 \\
\hline 6 & 0 & 0 & 0 & 0 & 0 & 0 & 0 & 0 & 0 & 0 & 0 & 0 \\
\hline 7 & 0 & 0 & 0 & 0 & 0 & 0 & 0 & 0 & 0 & 0 & 0 & 0 \\
\hline 8 & 0 & 0 & 0 & 0 & 0 & 0 & 0 & 0 & 0 & 0 & 0 & 0 \\
\hline 9 & 0 & 0 & 0 & 0 & 0 & 0 & 0 & 0 & 0 & 0 & 0 & 0 \\
\hline 10 & 0 & 0 & 0 & 0 & 0 & 0 & 0 & 0 & 0 & 0 & 0 & 0 \\
\hline 11 & 0 & 0 & 0 & 0 & 0 & 0 & 0 & 0 & 0 & 0 & 0 & 0 \\
\hline 12 & 0 & 0 & 0 & 0 & 0 & 0 & 0 & 0 & 0 & 0 & 0 & 0 \\
\hline 13 & 0 & 0 & 0 & 0 & 0 & 0 & 0 & 0 & 0 & 0 & 0 & 0 \\
\hline 14 & 0 & 0 & 0 & 0 & 0 & 0 & 0 & 0 & 0 & 0 & 0 & 0 \\
\hline 15 & 0 & 0 & 0 & 0 & 0 & 0 & 0 & 0 & 0 & 0 & 0 & 0 \\
\hline 16 & 0 & 0 & 0 & 0 & 0 & 0 & 0 & 0 & 0 & 0 & 0 & 0 \\
\hline 17 & 0 & 0 & 0 & 0 & 0 & 0 & 0 & 0 & 0 & 0 & 0 & 0 \\
\hline 18 & 0 & 0 & 0 & 0 & 0 & 0 & 0 & 0 & 0 & 0 & 0 & 0 \\
\hline 19 & 0 & 0 & 0 & 0 & 0 & 0 & 0 & 0 & 0 & 0 & 0 & 0 \\
\hline 20 & 0 & 0 & 0 & 0 & 0 & 0 & 0 & 0 & 0 & 0 & 0 & 0 \\
\hline 21 & 0 & 0 & 0 & 0 & 0 & 0 & 0 & 0 & 0 & 0 & 0 & 0 \\
\hline 22 & 0 & 0 & 0 & 0 & 0 & 0 & 0 & 0 & 0 & 0 & 0 & 0 \\
\hline 23 & 0 & 0 & 0 & 0 & 0 & 0 & 0 & 0 & 0 & 0 & 0 & 0 \\
\hline 24 & 0 & 0 & 0 & 0 & 0 & 0 & 0 & 0 & 0 & 0 & 0 & 0 \\
\hline 25 & 0 & 0 & 0 & 0 & 0 & 0 & 0 & 0 & 0 & 0 & 0 & 0 \\
\hline 26 & 0 & 0 & 0 & 0 & 0 & 0 & 0 & 0 & 0 & 0 & 0 & 0 \\
\hline 27 & 0 & 0 & 0 & 0 & 0 & 0 & 0 & 0 & 0 & 0 & 0 & 0 \\
\hline 28 & 0 & 0 & 0 & 0 & 0 & 0 & 0 & 0 & 0 & 0 & 0 & 0 \\
\hline 29 & 0 & 0 & 0 & 0 & ----- & 0 & 0 & 0 & 0 & 0 & 0 & 0 \\
\hline 30 & 0 & 0 & 0 & 0 & ------ & 0 & 0 & 0 & 0 & 0 & 0 & 0 \\
\hline 31 & 0 & ------ & 0 & 0 & ------ & 0 & ------ & 0 & ------ & 0 & 0 & ------ \\
\hline Total & 0 & 0 & 0 & 0 & 0 & 0 & 0 & 0 & 0 & 0 & 0 & 0 \\
\hline Mean & 0 & 0 & 0 & 0 & 0 & 0 & 0 & 0 & 0 & 0 & 0 & 0 \\
\hline Max & 0 & 0 & 0 & 0 & 0 & 0 & 0 & 0 & 0 & 0 & 0 & 0 \\
\hline Min & 0 & 0 & 0 & 0 & 0 & 0 & 0 & 0 & 0 & 0 & 0 & 0 \\
\hline $\mathrm{Ac}-\mathrm{Ft}$ & 0 & 0 & 0 & 0 & 0 & 0 & 0 & 0 & 0 & 0 & 0 & 0 \\
\hline Wtr Year & 2000 & Total & 0 & & Mean & 0 & Max & 0 & Min & 0 & $\mathrm{Ac}-\mathrm{Ft}$ & 0 \\
\hline Cal Year & 1999 & Total & 0 & & Mean & 0 & $\operatorname{Max}$ & 0 & Min & 0 & Ac-Ft & 0 \\
\hline
\end{tabular}




\section{E225 Cañada del Buey above White Rock, NM}

Location. Lat 35 50'1.3", long 106²14'22.1", in Ramon Vigil Grant, Los Alamos County, $0.1 \mathrm{mi}$ south of Santa Fe/Los Alamos County Line and 2.5 mi upstream from NM State Highway 4 in White Rock.

Drainage Area. $1.58 \mathrm{mi}^{2}$.

Period of Record. October 1993 to September 30, 2000.

Gage. Data logger with cellular telemetry and concrete control. Elevation of gage is 6,602 ft above National Geodetical Vertical Datum of 1929, from GPS survey.

Remarks. Water discharge records fair.

Extremes for Period of Record. Maximum discharge $17 \mathrm{ft}^{3} / \mathrm{s}$, September 8, 1995, gage height $2.71 \mathrm{ft}$. No flow most of time.

Extremes for Current Water Year. No flow for year.

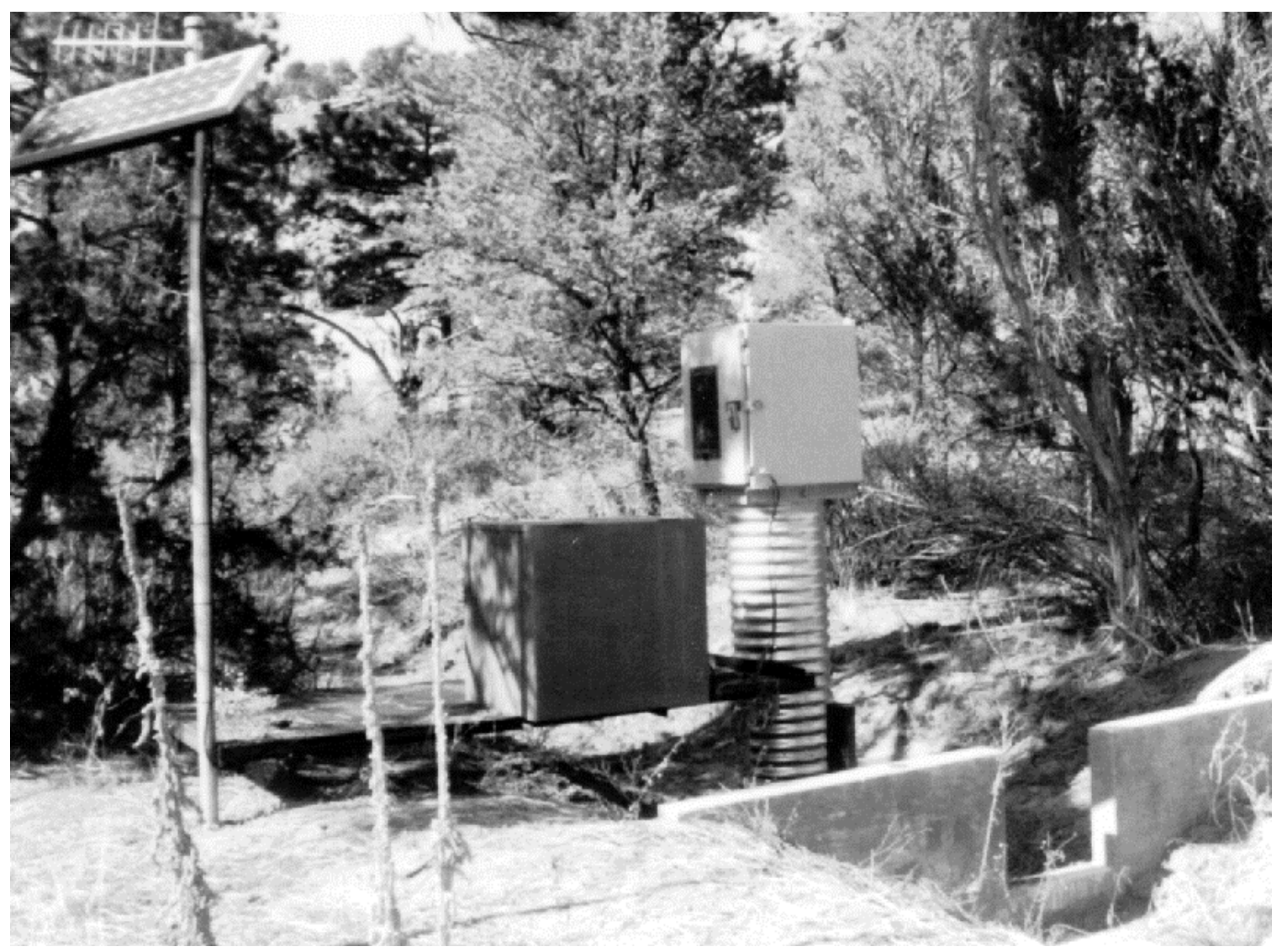




\section{E225 Cañada del Buey above White Rock, NM}

Daily Mean Discharge in Cubic Feet per Second

Water Year October 1999 to September 2000

\begin{tabular}{|c|c|c|c|c|c|c|c|c|c|c|c|c|}
\hline DAY & OCT & NOV & DEC & JAN & FEB & MAR & APR & MAY & JUN & JUL & AUG & SEP \\
\hline 1 & 0 & 0 & 0 & 0 & 0 & 0 & 0 & 0 & 0 & 0 & 0 & 0 \\
\hline 2 & 0 & 0 & 0 & 0 & 0 & 0 & 0 & 0 & 0 & 0 & 0 & 0 \\
\hline 3 & 0 & 0 & 0 & 0 & 0 & 0 & 0 & 0 & 0 & 0 & 0 & 0 \\
\hline 4 & 0 & 0 & 0 & 0 & 0 & 0 & 0 & 0 & 0 & 0 & 0 & 0 \\
\hline 5 & 0 & 0 & 0 & 0 & 0 & 0 & 0 & 0 & 0 & 0 & 0 & 0 \\
\hline 6 & 0 & 0 & 0 & 0 & 0 & 0 & 0 & 0 & 0 & 0 & 0 & 0 \\
\hline 7 & 0 & 0 & 0 & 0 & 0 & 0 & 0 & 0 & 0 & 0 & 0 & 0 \\
\hline 8 & 0 & 0 & 0 & 0 & 0 & 0 & 0 & 0 & 0 & 0 & 0 & 0 \\
\hline 9 & 0 & 0 & 0 & 0 & 0 & 0 & 0 & 0 & 0 & 0 & 0 & 0 \\
\hline 10 & 0 & 0 & 0 & 0 & 0 & 0 & 0 & 0 & 0 & 0 & 0 & 0 \\
\hline 11 & 0 & 0 & 0 & 0 & 0 & 0 & 0 & 0 & 0 & 0 & 0 & 0 \\
\hline 12 & 0 & 0 & 0 & 0 & 0 & 0 & 0 & 0 & 0 & 0 & 0 & 0 \\
\hline 13 & 0 & 0 & 0 & 0 & 0 & 0 & 0 & 0 & 0 & 0 & 0 & 0 \\
\hline 14 & 0 & 0 & 0 & 0 & 0 & 0 & 0 & 0 & 0 & 0 & 0 & 0 \\
\hline 15 & 0 & 0 & 0 & 0 & 0 & 0 & 0 & 0 & 0 & 0 & 0 & 0 \\
\hline 16 & 0 & 0 & 0 & 0 & 0 & 0 & 0 & 0 & 0 & 0 & 0 & 0 \\
\hline 17 & 0 & 0 & 0 & 0 & 0 & 0 & 0 & 0 & 0 & 0 & 0 & 0 \\
\hline 18 & 0 & 0 & 0 & 0 & 0 & 0 & 0 & 0 & 0 & 0 & 0 & 0 \\
\hline 19 & 0 & 0 & 0 & 0 & 0 & 0 & 0 & 0 & 0 & 0 & 0 & 0 \\
\hline 20 & 0 & 0 & 0 & 0 & 0 & 0 & 0 & 0 & 0 & 0 & 0 & 0 \\
\hline 21 & 0 & 0 & 0 & 0 & 0 & 0 & 0 & 0 & 0 & 0 & 0 & 0 \\
\hline 22 & 0 & 0 & 0 & 0 & 0 & 0 & 0 & 0 & 0 & 0 & 0 & 0 \\
\hline 23 & 0 & 0 & 0 & 0 & 0 & 0 & 0 & 0 & 0 & 0 & 0 & 0 \\
\hline 24 & 0 & 0 & 0 & 0 & 0 & 0 & 0 & 0 & 0 & 0 & 0 & 0 \\
\hline 25 & 0 & 0 & 0 & 0 & 0 & 0 & 0 & 0 & 0 & 0 & 0 & 0 \\
\hline 26 & 0 & 0 & 0 & 0 & 0 & 0 & 0 & 0 & 0 & 0 & 0 & 0 \\
\hline 27 & 0 & 0 & 0 & 0 & 0 & 0 & 0 & 0 & 0 & 0 & 0 & 0 \\
\hline 28 & 0 & 0 & 0 & 0 & 0 & 0 & 0 & 0 & 0 & 0 & 0 & 0 \\
\hline 29 & 0 & 0 & 0 & 0 & ----- & 0 & 0 & 0 & 0 & 0 & 0 & 0 \\
\hline 30 & 0 & 0 & 0 & 0 & ------ & 0 & 0 & 0 & 0 & 0 & 0 & 0 \\
\hline 31 & 0 & ------ & 0 & 0 & ------ & 0 & ------ & 0 & ------ & 0 & 0 & ------ \\
\hline Total & 0 & 0 & 0 & 0 & 0 & 0 & 0 & 0 & 0 & 0 & 0 & 0 \\
\hline Mean & 0 & 0 & 0 & 0 & 0 & 0 & 0 & 0 & 0 & 0 & 0 & 0 \\
\hline Max & 0 & 0 & 0 & 0 & 0 & 0 & 0 & 0 & 0 & 0 & 0 & 0 \\
\hline Min & 0 & 0 & 0 & 0 & 0 & 0 & 0 & 0 & 0 & 0 & 0 & 0 \\
\hline $\mathrm{Ac}-\mathrm{Ft}$ & 0 & 0 & 0 & 0 & 0 & 0 & 0 & 0 & 0 & 0 & 0 & 0 \\
\hline Wtr Year & 2000 & Total & 0 & & Mean & 0 & Max & 0 & Min & 0 & $\mathrm{Ac}-\mathrm{Ft}$ & 0 \\
\hline Cal Year & 1999 & Total & 0 & & Mean & 0 & $\operatorname{Max}$ & 0 & Min & 0 & Ac-Ft & 0 \\
\hline
\end{tabular}




\section{E230 Cañada del Buey at White Rock, NM}

Location. Lat 35'49'38", long 106²'43", in Ramon Vigil Grant, Los Alamos County, $250 \mathrm{ft}$ upstream from NM State Highway 4 in White Rock, NM.

Drainage Area. $2.14 \mathrm{mi}^{2}$.

Period of Record. October 1991 to September 30, 2000.

Gage. Data logger with cellular telemetry and concrete control. Elevation of gage is 6,401 ft above National Geodetic Vertical Datum of 1929, from GPS survey.

Remarks. Water discharge records fair.

Extremes for Period of Record. Maximum discharge $210 \mathrm{ft}^{3} / \mathrm{s}$, June 17, 1999, gage height $3.30 \mathrm{ft}$. No flow most of the time.

Extremes for Current Water Year. Maximum discharge $33 \mathrm{ft}^{3} / \mathrm{s}$ at $2020 \mathrm{hrs,} \mathrm{August} \mathrm{9,} \mathrm{gage}$ height $1.37 \mathrm{ft}$. No flow most of the time.

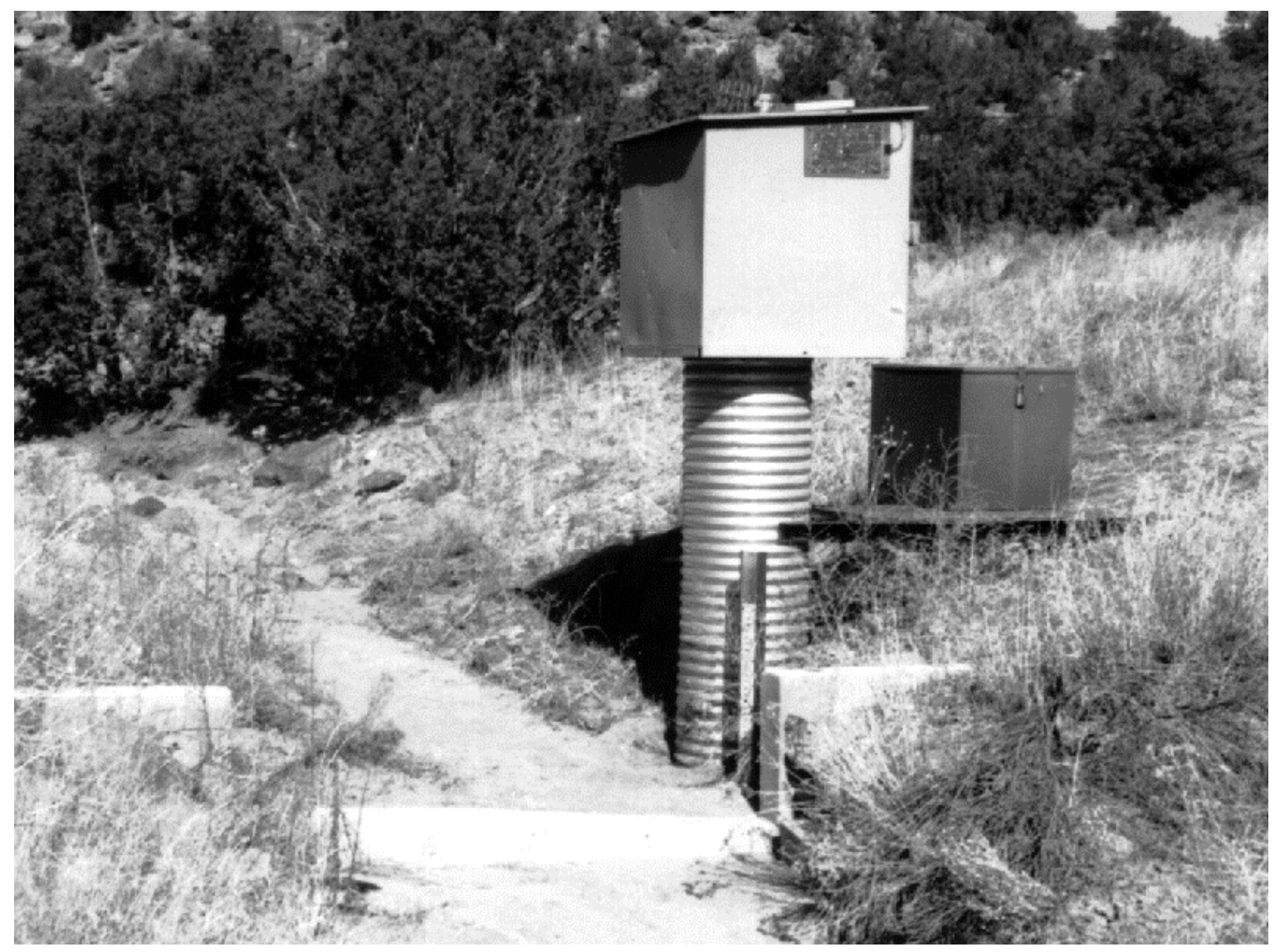




\section{E230 Cañada del Buey at White Rock, NM}

Daily Mean Discharge in Cubic Feet per Second

Water Year October 1999 to September 2000

\begin{tabular}{|c|c|c|c|c|c|c|c|c|c|c|c|c|}
\hline DAY & OCT & NOV & DEC & JAN & FEB & MAR & APR & MAY & JUN & JUL & AUG & SEP \\
\hline 1 & 0 & 0 & 0 & 0 & 0 & 0 & 0 & 0 & 0 & 0 & 0 & 0 \\
\hline 2 & 0 & 0 & 0 & 0 & 0 & 0 & 0 & 0 & 0 & 0 & 0 & 0 \\
\hline 3 & 0 & 0 & 0 & 0 & 0 & 0 & 0 & 0 & 0 & 0 & 0 & 0 \\
\hline 4 & 0 & 0 & 0 & 0 & 0 & 0 & 0 & 0 & 0 & 0 & 0 & 0 \\
\hline 5 & 0 & 0 & 0 & 0 & 0 & 0 & 0 & 0 & 0 & 0 & 0 & 0 \\
\hline 6 & 0 & 0 & 0 & 0 & 0 & 0 & 0 & 0 & 0 & 0 & 0 & 0 \\
\hline 7 & 0 & 0 & 0 & 0 & 0 & 0 & 0 & 0 & 0 & 0 & 0 & 0 \\
\hline 8 & 0 & 0 & 0 & 0 & 0 & 0 & 0 & 0 & 0 & 0 & .72 & 0 \\
\hline 9 & 0 & 0 & 0 & 0 & 0 & 0 & 0 & 0 & 0 & 0 & 0 & 0 \\
\hline 10 & 0 & 0 & 0 & 0 & 0 & 0 & 0 & 0 & 0 & 0 & 0 & 0 \\
\hline 11 & 0 & 0 & 0 & 0 & 0 & 0 & 0 & 0 & 0 & 0 & 0 & 0 \\
\hline 12 & 0 & 0 & 0 & 0 & 0 & 0 & 0 & 0 & 0 & 0 & 0 & 0 \\
\hline 13 & 0 & 0 & 0 & 0 & 0 & 0 & 0 & 0 & 0 & 0 & 0 & 0 \\
\hline 14 & 0 & 0 & 0 & 0 & 0 & 0 & 0 & 0 & 0 & 0 & 0 & 0 \\
\hline 15 & 0 & 0 & 0 & 0 & 0 & 0 & 0 & 0 & 0 & 0 & 0 & 0 \\
\hline 16 & 0 & 0 & 0 & 0 & 0 & 0 & 0 & 0 & 0 & 0 & 0 & 0 \\
\hline 17 & 0 & 0 & 0 & 0 & 0 & 0 & 0 & 0 & 0 & 0 & 0 & 0 \\
\hline 18 & 0 & 0 & 0 & 0 & 0 & 0 & 0 & 0 & 0 & 0 & .36 & 0 \\
\hline 19 & 0 & 0 & 0 & 0 & 0 & 0 & 0 & 0 & 0 & 0 & .13 & 0 \\
\hline 20 & 0 & 0 & 0 & 0 & 0 & 0 & 0 & 0 & 0 & 0 & 0 & 0 \\
\hline 21 & 0 & 0 & 0 & 0 & 0 & 0 & 0 & 0 & 0 & 0 & 0 & 0 \\
\hline 22 & 0 & 0 & 0 & 0 & 0 & 0 & 0 & 0 & 0 & 0 & 0 & 0 \\
\hline 23 & 0 & 0 & 0 & 0 & 0 & 0 & 0 & 0 & 0 & 0 & 0 & 0 \\
\hline 24 & 0 & 0 & 0 & 0 & 0 & 0 & 0 & 0 & 0 & 0 & 0 & 0 \\
\hline 25 & 0 & 0 & 0 & 0 & 0 & 0 & 0 & 0 & 0 & 0 & 0 & 0 \\
\hline 26 & 0 & 0 & 0 & 0 & 0 & 0 & 0 & 0 & 0 & 0 & 0 & 0 \\
\hline 27 & 0 & 0 & 0 & 0 & 0 & 0 & 0 & 0 & 0 & 0 & 0 & 0 \\
\hline 28 & 0 & 0 & 0 & 0 & 0 & 0 & 0 & 0 & 0 & 0 & 0 & 0 \\
\hline 29 & 0 & 0 & 0 & 0 & ------ & 0 & 0 & 0 & 0 & .08 & 0 & 0 \\
\hline 30 & 0 & 0 & 0 & 0 & ----- & 0 & 0 & 0 & .01 & 0 & 0 & 0 \\
\hline 31 & 0 & ------ & 0 & 0 & ------ & 0 & -.---. & 0 & ------ & 0 & 0 & --.-- \\
\hline Total & 0 & 0 & 0 & 0 & 0 & 0 & 0 & 0 & 0.01 & 0.08 & 1.21 & 0 \\
\hline Mean & 0 & 0 & 0 & 0 & 0 & 0 & 0 & 0 & 0 & .003 & .039 & 0 \\
\hline Max & 0 & 0 & 0 & 0 & 0 & 0 & 0 & 0 & .01 & .08 & .72 & 0 \\
\hline Min & 0 & 0 & 0 & 0 & 0 & 0 & 0 & 0 & 0 & 0 & 0 & 0 \\
\hline $\mathrm{Ac}-\mathrm{Ft}$ & 0 & 0 & 0 & 0 & 0 & 0 & 0 & 0 & .02 & .2 & 2.4 & 0 \\
\hline Wtr Year & 2000 & Total & 1. & & Mean & .004 & Max & .72 & Min & 0 & $\mathrm{Ac}-\mathrm{Ft}$ & 2.6 \\
\hline Cal Year & 1999 & Total & 10. & & Mean & .030 & Max & 5.9 & Min & 0 & $\mathrm{Ac}-\mathrm{Ft}$ & 22 \\
\hline
\end{tabular}




\section{E240 Pajarito Canyon above Highway 501 near Los Alamos, NM}

Location. Lat 3552'3.9", long 106²1'09", SE 1/4 NW 1/4, sec. 19, T. 19 N, R. 6 E, Los Alamos County, in Santa Fe National Forest, $200 \mathrm{ft}$ upstream from NM State Highway 501.

Drainage Area. $1.90 \mathrm{mi}^{2}$.

Period of Record. October 1993 to September 30, 2000.

Gage. Data logger with cellular telemetry and Parshall Flume. Elevation of gage is 7,740 $\mathrm{ft}$ above National Geodetic Vertical Datum of 1929, from GPS survey.

Revisions. Gage height “Extremes for Period of Record” WDR 1997 (gage height).

Remarks. Records poor. Gage destroyed by flood June 28.

Extremes for Period of Record. Maximum discharge 1,020 ft $3 / \mathrm{s}$, June 28, 2000, from peak flow computation, gage height not determined. No flow at times.

Extremes for Current Water Year. Maximum discharge $1,020 \mathrm{ft}^{3} / \mathrm{s}$ at about $1230 \mathrm{hrs,} \mathrm{June} \mathrm{28,}$ from peak flow computation, gage height not determined. No flow at times.

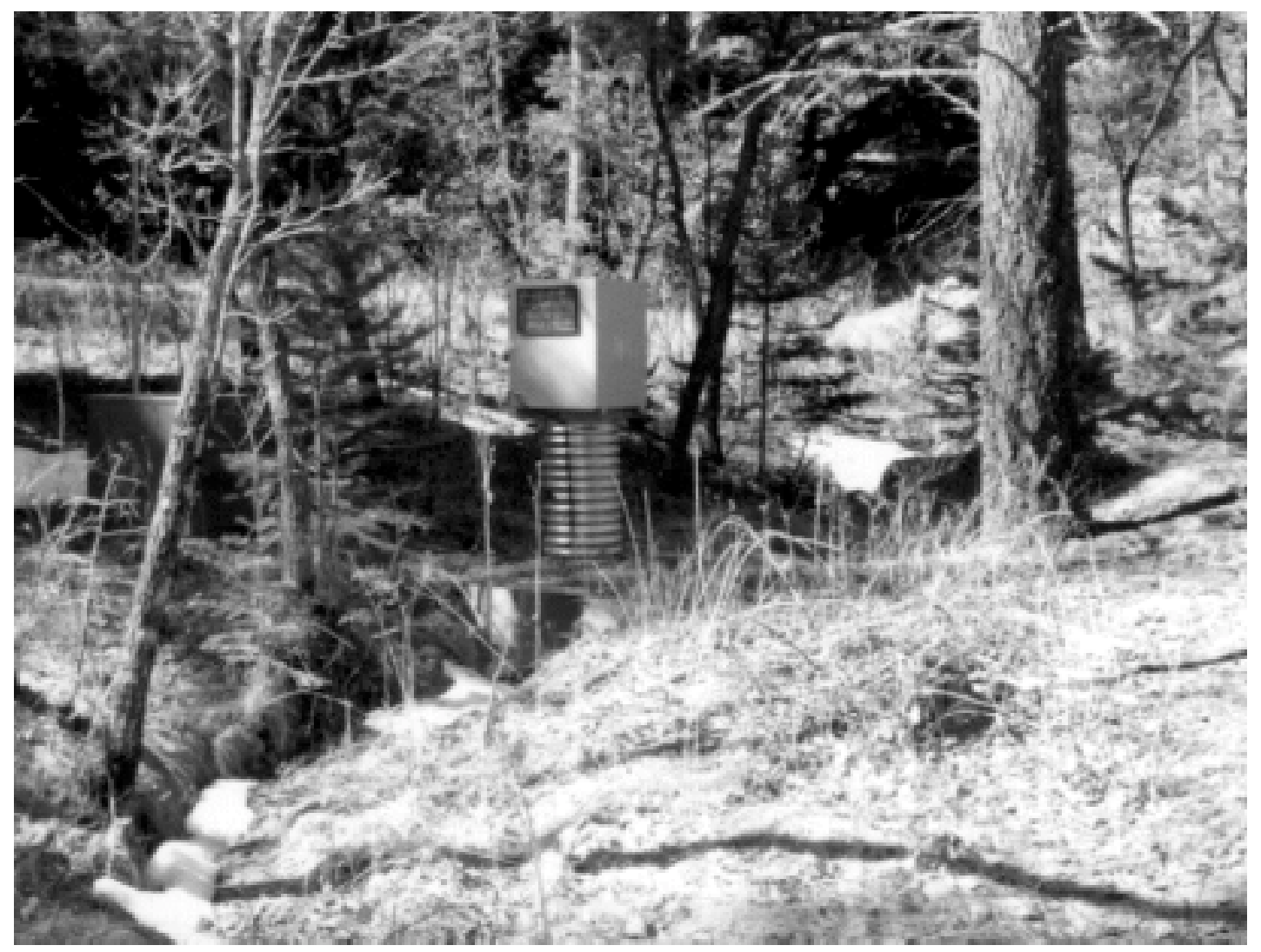


E240 Pajarito Canyon above Highway 501 near Los Alamos, NM

Daily Mean Discharge in Cubic Feet per Second

Water Year October 1999 to September 2000

\begin{tabular}{|c|c|c|c|c|c|c|c|c|c|c|c|c|}
\hline DAY & OCT & NOV & DEC & JAN & FEB & MAR & APR & MAY & JUN & JUL & AUG & SEP \\
\hline 1 & 0 & 0 & .03 & 0 & 0 & 0 & 0 & 0 & 0 & & & \\
\hline 2 & 0 & 0 & .06 & 0 & 0 & 0 & 0 & 0 & 1.8 & & & \\
\hline 3 & 0 & 0 & .07 & 0 & 0 & 0 & 0 & 0 & .25 & & & \\
\hline 4 & 0 & 0 & .12 & 0 & 0 & 0 & 0 & 0 & .10 & & & \\
\hline 5 & 0 & 0 & .09 & 0 & 0 & 0 & 0 & 0 & 0 & & & \\
\hline 6 & 0 & 0 & .05 & 0 & 0 & 0 & 0 & 0 & 0 & & & \\
\hline 7 & 0 & 0 & .02 & 0 & 0 & 0 & 0 & 0 & 0 & & & \\
\hline 8 & 0 & 0 & 0 & 0 & 0 & 0 & 0 & 0 & 0 & & & \\
\hline 9 & 0 & 0 & 0 & 0 & 0 & 0 & 0 & 0 & 0 & & & \\
\hline 10 & 0 & 0 & 0 & 0 & 0 & 0 & 0 & 0 & 0 & & & \\
\hline 11 & 0 & 0 & 0 & 0 & 0 & 0 & 0 & 0 & 0 & & & \\
\hline 12 & 0 & 0 & 0 & 0 & 0 & 0 & 0 & 0 & 0 & & & \\
\hline 13 & 0 & 0 & 0 & 0 & 0 & 0 & 0 & 0 & 0 & & & \\
\hline 14 & 0 & 0 & 0 & 0 & 0 & 0 & 0 & 0 & 0 & & & \\
\hline 15 & 0 & 0 & 0 & 0 & 0 & 0 & 0 & 0 & 0 & & & \\
\hline 16 & 0 & 0 & 0 & 0 & 0 & 0 & 0 & 0 & 0 & & & \\
\hline 17 & 0 & 0 & 0 & 0 & 0 & 0 & 0 & 0 & 0 & & & \\
\hline 18 & 0 & .01 & 0 & 0 & 0 & 0 & 0 & 0 & 0 & & & \\
\hline 19 & 0 & .08 & 0 & 0 & 0 & 0 & 0 & 0 & 0 & & & \\
\hline 20 & 0 & .14 & 0 & 0 & 0 & 0 & 0 & 0 & 0 & & & \\
\hline 21 & 0 & 0 & 0 & 0 & 0 & 0 & 0 & 0 & 0 & & & \\
\hline 22 & 0 & .04 & 0 & 0 & 0 & 0 & 0 & 0 & 0 & & & \\
\hline 23 & 0 & .11 & 0 & 0 & 0 & 0 & 0 & 0 & 0 & & & \\
\hline 24 & 0 & .17 & 0 & 0 & 0 & 0 & 0 & 0 & 0 & & & \\
\hline 25 & 0 & .14 & 0 & 0 & 0 & 0 & 0 & 0 & 0 & & & \\
\hline 26 & 0 & .12 & 0 & 0 & 0 & 0 & 0 & 0 & 0 & & & \\
\hline 27 & 0 & .03 & 0 & 0 & 0 & 0 & 0 & 0 & 0 & & & \\
\hline 28 & 0 & .02 & 0 & 0 & 0 & 0 & 0 & 0 & 24 & & & \\
\hline 29 & 0 & .01 & 0 & 0 & ------ & 0 & 0 & 0 & 1.0 & & & \\
\hline 30 & 0 & .03 & 0 & 0 & ------ & 0 & 0 & 0 & .25 & & & \\
\hline 31 & 0 & ----- & 0 & 0 & ----- & 0 & ----- & 0 & ----- & & & \\
\hline Total & 0 & 0.90 & 0.44 & 0 & 0 & 0 & 0 & 0 & 27.40 & & & \\
\hline Mean & 0 & .030 & .014 & 0 & 0 & 0 & 0 & 0 & .91 & & & \\
\hline Max & 0 & .17 & .12 & 0 & 0 & 0 & 0 & 0 & 24 & & & \\
\hline Min & 0 & 0 & 0 & 0 & 0 & 0 & 0 & 0 & 0 & & & \\
\hline Ac-Ft & 0 & 1.8 & .8 & 0 & 0 & 0 & 0 & 0 & 55 & & & \\
\hline Wtr Year & 2000 & Toté & & & Mean & .10 & Max & 24 & Min & 0 & $\mathrm{Ac}-\mathrm{Ft}$ & 57 \\
\hline Cal Year & 1999 & Totá & & & Mean & .055 & $\operatorname{Max}$ & .51 & Min & 0 & Ac-Ft & 40 \\
\hline
\end{tabular}




\section{E241 Pajarito Canyon at TA-22}

Location. Lat 3551'33.6", long 106²0'12.6", SW 1/4, SW 1/4 sec. 20, T. 19 N, R. 6 E, Los Alamos County, $100 \mathrm{ft}$ upstream from mouth of Starmers Gulch (E242), 0.5 mi S of LANL TA-22 building 91 .

Drainage Area. $3.97 \mathrm{mi}^{2}$.

Period of Record. March 1999 to September 30, 2000.

Gage. Data logger with cellular telemetry and $90^{\circ}$ sharp crested weir. Elevation of gage is 7,382 ft above National Geodetic Vertical Datum of 1929, from GPS survey.

Remarks. Records poor. Gage destroyed by flood June 28.

Extremes for Period of Record. Maximum discharge, $300 \mathrm{ft}^{3} / \mathrm{s}$, June 28, 2000, from peak flow computation, gage height $5.00 \mathrm{ft}$. No flow at times.

Extremes for Current Water Year. Maximum discharge, $300 \mathrm{ft}^{3} / \mathrm{s}$ at about $1250 \mathrm{hrs}$, June 28, from peak flow computation, gage height $5.00 \mathrm{ft}$. No flow at times.

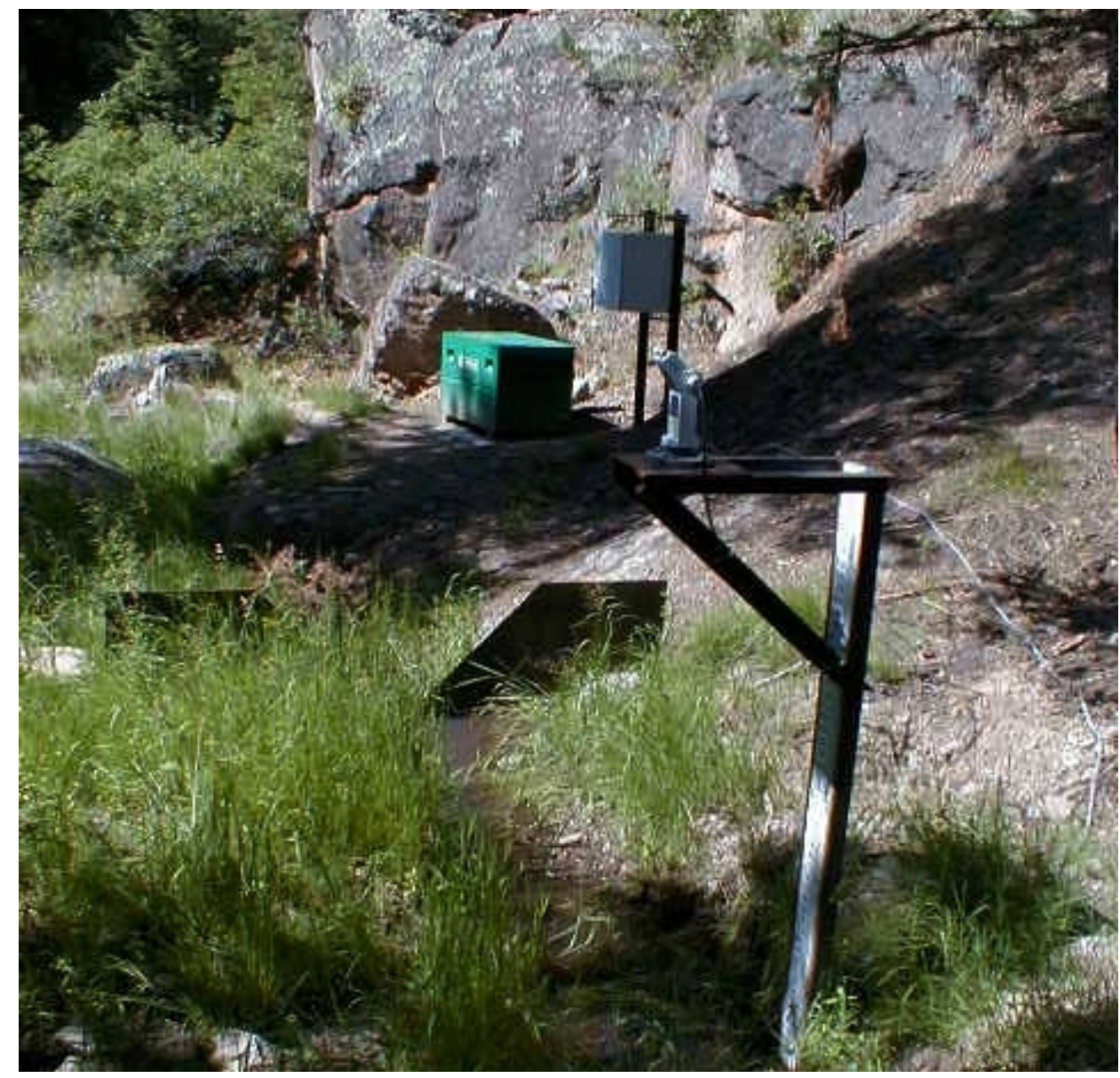




\section{E241 Pajarito Canyon at TA-22}

Daily Mean Discharge in Cubic Feet per Second

Water Year October 1999 to September 2000

\begin{tabular}{|c|c|c|c|c|c|c|c|c|c|c|c|c|}
\hline DAY & OCT & NOV & DEC & JAN & FEB & MAR & APR & MAY & JUN & JUL & AUG & SEP \\
\hline 1 & .04 & .02 & .02 & .59 & .24 & .02 & .04 & .04 & .07 & .09 & & \\
\hline 2 & .03 & .02 & .01 & .58 & .23 & .02 & .04 & .03 & 1.0 & .05 & & \\
\hline 3 & .03 & .02 & .02 & .57 & .18 & .02 & .05 & .02 & .30 & .02 & & \\
\hline 4 & .03 & .02 & .01 & .91 & .17 & .02 & .04 & .02 & .08 & & & \\
\hline 5 & .03 & .03 & .02 & 1.0 & .16 & .03 & .04 & .02 & .08 & & & \\
\hline 6 & .03 & .03 & .02 & 1.0 & .15 & .03 & .04 & .01 & .09 & & & \\
\hline 7 & .03 & .03 & .02 & 1.2 & .13 & .03 & .04 & .02 & .09 & & & \\
\hline 8 & .03 & .03 & .02 & 1.2 & .13 & .02 & .05 & .02 & .09 & & & \\
\hline 9 & .03 & .02 & .04 & 1.3 & .11 & .02 & .05 & .02 & .10 & & & \\
\hline 10 & .02 & .02 & .03 & 1.3 & .09 & .02 & .05 & .02 & .10 & & & \\
\hline 11 & .02 & .02 & .02 & 1.2 & .06 & .02 & .06 & .01 & .10 & & & \\
\hline 12 & .02 & .02 & .04 & 1.2 & .03 & .02 & .05 & .02 & .10 & & & \\
\hline 13 & .02 & .02 & .08 & 1.1 & .02 & .02 & .04 & .03 & .11 & & & \\
\hline 14 & .02 & .02 & .08 & 1.1 & .02 & .02 & .04 & .03 & .11 & & & \\
\hline 15 & .02 & .02 & .12 & 1.1 & .02 & .02 & .05 & .03 & .11 & & & \\
\hline 16 & .03 & .02 & .19 & 1.0 & .02 & .03 & .05 & .03 & .12 & & & \\
\hline 17 & .03 & .02 & .20 & .96 & .02 & .02 & .05 & .03 & .12 & & & \\
\hline 18 & .03 & .01 & .21 & .83 & .01 & .02 & .05 & .04 & .12 & & & \\
\hline 19 & .03 & .01 & .21 & .72 & .02 & .03 & .05 & .04 & .13 & & & \\
\hline 20 & .03 & .02 & .29 & .68 & .02 & .03 & .05 & .04 & .20 & & & \\
\hline 21 & .03 & .02 & .31 & .61 & .02 & .03 & .06 & .04 & .30 & & & \\
\hline 22 & .02 & .02 & .45 & .53 & .02 & .04 & .05 & .04 & .31 & & & \\
\hline 23 & .03 & .03 & .65 & .49 & .02 & .03 & .05 & .05 & .29 & & & \\
\hline 24 & .03 & .02 & .71 & .45 & .02 & .03 & .06 & .05 & .31 & & & \\
\hline 25 & .02 & .03 & .71 & .40 & .02 & .03 & .05 & .05 & .31 & & & \\
\hline 26 & .02 & .02 & .71 & .35 & .03 & .03 & .05 & .05 & .31 & & & \\
\hline 27 & .02 & .02 & .68 & .29 & .02 & .03 & .05 & .06 & .30 & & & \\
\hline 28 & .02 & .02 & .62 & .26 & .02 & .04 & .05 & .06 & 3.3 & & & \\
\hline 29 & .02 & .02 & .60 & .24 & ----- & .04 & .04 & .06 & .49 & & & \\
\hline 30 & .02 & .02 & .60 & .24 & ----- & .04 & .04 & .07 & .21 & & & \\
\hline 31 & .02 & ------ & .59 & .24 & ----- & .05 & ------ & .07 & ------ & & & \\
\hline Total & 0.80 & 0.64 & 8.28 & 23.64 & 2.01 & 0.85 & 1.43 & 1.12 & 9.35 & 0.16 & & \\
\hline Mean & .026 & .021 & .27 & .76 & .069 & .027 & .048 & .036 & .31 & .006 & & \\
\hline Max & .04 & .03 & .71 & 1.3 & .24 & .05 & .06 & .07 & 3.3 & .09 & & \\
\hline Min & .02 & .01 & .01 & .24 & .01 & .02 & .04 & .01 & .07 & 0 & & \\
\hline$A c-F t$ & 1.5 & 1.2 & 16 & 47 & 4.0 & 1.6 & 2.8 & 2.0 & 19 & .3 & & \\
\hline Wtr Year & 2000 & Tota & & 28 & lean & .16 & Max & 3.3 & Min & 0 & $\mathrm{Ac}-\mathrm{Ft}$ & 95 \\
\hline Cal Year & 1999 & Tota & & 42 & lean & .061 & Max & .71 & Min & 0 & $\mathrm{Ac}-\mathrm{Ft}$ & 34 \\
\hline
\end{tabular}




\section{E242 Starmer's Gulch at TA-22}

Location. Lat 3551'33.0", long 106²0'13.0", SW 1/4, SW 1/4 sec. 20, T. 19 N, R. 6 E, Los Alamos County, $100 \mathrm{ft}$ upstream from confluence of Starmer's Gulch and Pajarito Canyon, $0.5 \mathrm{mi} \mathrm{S}$ of LANL TA-22, building 91.

Drainage Area. $0.82 \mathrm{mi}^{2}$.

Period of Record. March 1999 to September 30, 2000.

Gage. Data logger with cellular telemetry. Elevation of gage is 7,390 ft above National Geodetic Vertical Datum of 1929, from GPS survey.

Remarks. Water discharge records fair.

Extremes for Period of Record. Maximum discharge $180 \mathrm{ft}^{3} / \mathrm{s}$, June 28, 2000, gage height $2.75 \mathrm{ft}$. Minimum daily $0.01 \mathrm{ft}^{3} / \mathrm{s}$, many days.

Extremes for March 1999-September 2000. Maximum discharge $180 \mathrm{ft}^{3} / \mathrm{s}$, at $1240 \mathrm{hrs,}$ June 28, gage height $2.75 \mathrm{ft}$. Minimum daily $0.01 \mathrm{ft}^{3} / \mathrm{s}$, many days.

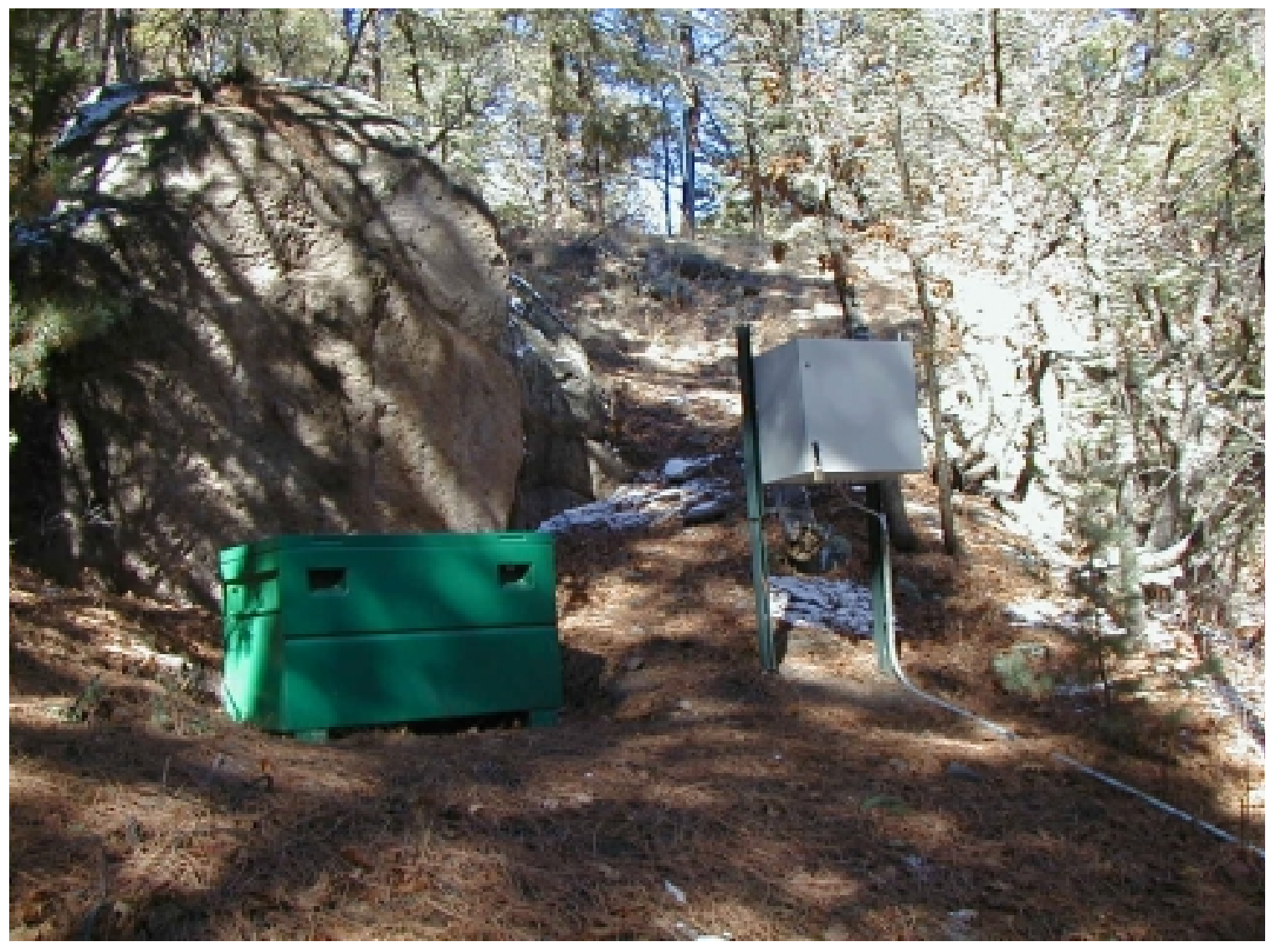


E242 Starmer's Gulch at TA-22

Daily Mean Discharge in Cubic Feet per Second

Water Year October 1998 to September 1999

\begin{tabular}{|c|c|c|c|c|c|c|c|c|c|c|c|c|}
\hline DAY & ОСТ & NOV & DEC & JAN & FEB & MAR & APR & MAY & JUN & JUL & AUG & SEP \\
\hline 1 & & & & & & & .05 & .39 & .18 & .03 & .01 & .05 \\
\hline 2 & & & & & & & .06 & .54 & .17 & .03 & .01 & .05 \\
\hline 3 & & & & & & & .08 & .74 & .15 & .03 & .02 & .05 \\
\hline 4 & & & & & & & .08 & .77 & .14 & .03 & .03 & .05 \\
\hline 5 & & & & & & & .08 & .74 & .13 & .03 & .04 & .05 \\
\hline 6 & & & & & & & .08 & .68 & .12 & .08 & .04 & .05 \\
\hline 7 & & & & & & & .09 & .60 & .12 & .04 & .04 & .05 \\
\hline 8 & & & & & & & .11 & .52 & .11 & .04 & .03 & .05 \\
\hline 9 & & & & & & & .14 & .45 & .10 & .06 & .03 & .04 \\
\hline 10 & & & & & & & .16 & .37 & .09 & .05 & .03 & .04 \\
\hline 11 & & & & & & & .16 & .31 & .09 & .04 & .03 & .04 \\
\hline 12 & & & & & & & .16 & .27 & .08 & .03 & .02 & .04 \\
\hline 13 & & & & & & & .15 & .24 & .08 & .03 & .02 & .04 \\
\hline 14 & & & & & & & .13 & .22 & .08 & .03 & .02 & .16 \\
\hline 15 & & & & & & & .12 & .20 & .08 & .03 & .03 & .03 \\
\hline 16 & & & & & & & .12 & .18 & .08 & .03 & .03 & .18 \\
\hline 17 & & & & & & & .12 & .16 & .10 & .02 & .05 & .06 \\
\hline 18 & & & & & & .03 & .10 & .15 & .11 & .03 & .08 & .04 \\
\hline 19 & & & & & & .04 & .09 & .14 & .10 & .02 & .08 & .04 \\
\hline 20 & & & & & & .04 & .09 & .14 & .09 & .03 & .07 & .03 \\
\hline 21 & & & & & & .04 & .08 & .13 & .11 & .02 & .07 & .02 \\
\hline 22 & & & & & & .05 & .08 & .12 & .08 & .02 & .07 & .02 \\
\hline 23 & & & & & & .06 & .08 & .12 & .06 & .02 & .07 & .01 \\
\hline 24 & & & & & & .06 & .09 & .12 & .05 & .01 & .06 & .01 \\
\hline 25 & & & & & & .05 & .15 & .13 & .05 & .01 & .06 & .01 \\
\hline 26 & & & & & & .05 & .13 & .14 & .04 & .01 & .06 & .01 \\
\hline 27 & & & & & & .05 & .15 & .15 & .04 & .01 & .06 & .01 \\
\hline 28 & & & & & & .06 & .16 & .25 & .04 & .01 & .05 & .01 \\
\hline 29 & & & & & & .06 & .16 & .20 & .04 & .02 & .05 & .01 \\
\hline 30 & & & & & & .06 & .46 & .20 & .03 & .02 & .06 & .01 \\
\hline 31 & & & & & & .05 & ----- & .20 & ----- & .01 & .06 & ----- \\
\hline Total & & & & & & 0.70 & 3.71 & 9.57 & 2.74 & 0.87 & 1.38 & 1.26 \\
\hline Mean & & & & & & .050 & .12 & .31 & .091 & .028 & .045 & .042 \\
\hline Max & & & & & & .06 & .46 & .77 & .18 & .08 & .08 & .18 \\
\hline Min & & & & & & .03 & .05 & .12 & .03 & .01 & .01 & .01 \\
\hline Ac-Ft & & & & & & 1.3 & 7.6 & 19 & 5.6 & 1.7 & 2.6 & 2.5 \\
\hline Wtr Year & 1999 & Total & 20.23 & & Mean & .10 & Max & .77 & Min & .01 & $\mathrm{Ac}-\mathrm{Ft}$ & 40 \\
\hline Cal Year & 1998 & Total & & & Mean & & Max & & Min & & $\mathrm{Ac}-\mathrm{Ft}$ & \\
\hline
\end{tabular}


E242 Starmer's Gulch at TA-22

Daily Mean Discharge in Cubic Feet per Second

Water Year October 1999 to September 2000

\begin{tabular}{|c|c|c|c|c|c|c|c|c|c|c|c|c|}
\hline DAY & ОСТ & NOV & DEC & JAN & FEB & MAR & APR & MAY & JUN & JUL & AUG & SEP \\
\hline 1 & .03 & .04 & .03 & .03 & .01 & .04 & .06 & .05 & .03 & .05 & .19 & .07 \\
\hline 2 & .03 & .04 & .03 & .03 & .01 & .04 & .06 & .04 & .03 & .05 & .19 & .08 \\
\hline 3 & .03 & .04 & .03 & .03 & .01 & .04 & .06 & .04 & .03 & .05 & .19 & .08 \\
\hline 4 & .03 & .04 & .03 & .03 & .01 & .04 & .06 & .04 & .03 & .05 & .21 & .07 \\
\hline 5 & .03 & .04 & .02 & .03 & .01 & .04 & .05 & .04 & .03 & .05 & .19 & .06 \\
\hline 6 & .03 & .04 & .02 & .03 & .01 & .04 & .05 & .04 & .03 & .05 & .20 & .06 \\
\hline 7 & .03 & .03 & .02 & .03 & .01 & .04 & .05 & .04 & .03 & .05 & .20 & .05 \\
\hline 8 & .03 & .03 & .02 & .03 & .01 & .04 & .05 & .04 & .03 & .05 & .20 & .05 \\
\hline 9 & .04 & .03 & .03 & .03 & .02 & .04 & .05 & .04 & .04 & .29 & .20 & .07 \\
\hline 10 & .04 & .03 & .03 & .03 & .02 & .04 & .05 & .04 & .04 & .04 & .21 & .08 \\
\hline 11 & .04 & .03 & .03 & .03 & .02 & .04 & .05 & .04 & .04 & .04 & .20 & .08 \\
\hline 12 & .03 & .03 & .03 & .03 & .02 & .04 & .05 & .04 & .04 & .04 & .21 & .07 \\
\hline 13 & .03 & .03 & .03 & .03 & .02 & .04 & .05 & .04 & .04 & .04 & .20 & .07 \\
\hline 14 & .03 & .03 & .03 & .02 & .02 & .04 & .05 & .04 & .04 & .04 & .20 & .06 \\
\hline 15 & .03 & .03 & .03 & .02 & .02 & .04 & .05 & .04 & .04 & .04 & .18 & .05 \\
\hline 16 & .03 & .03 & .03 & .02 & .02 & .04 & .05 & .04 & .04 & .04 & .16 & .05 \\
\hline 17 & .04 & .03 & .03 & .02 & .03 & .04 & .05 & .04 & .04 & .04 & .13 & .05 \\
\hline 18 & .04 & .03 & .03 & .02 & .03 & .04 & .05 & .04 & .04 & .04 & .21 & .05 \\
\hline 19 & .04 & .03 & .03 & .02 & .03 & .04 & .04 & .03 & .04 & .04 & .33 & .05 \\
\hline 20 & .04 & .03 & .03 & .02 & .03 & .04 & .04 & .03 & .04 & .04 & .33 & .05 \\
\hline 21 & .04 & .04 & .03 & .02 & .03 & .05 & .04 & .03 & .04 & .04 & .28 & .06 \\
\hline 22 & .04 & .04 & .03 & .02 & .03 & .05 & .04 & .03 & .04 & .04 & .25 & .06 \\
\hline 23 & .04 & .03 & .03 & .02 & .03 & .06 & .04 & .03 & .05 & .16 & .25 & .07 \\
\hline 24 & .04 & .03 & .03 & .02 & .03 & .07 & .04 & .03 & .06 & .15 & .21 & .06 \\
\hline 25 & .04 & .03 & .03 & .02 & .03 & .06 & .04 & .03 & .08 & .18 & .16 & .06 \\
\hline 26 & .04 & .02 & .03 & .02 & .03 & .06 & .04 & .03 & .09 & .22 & .13 & .06 \\
\hline 27 & .04 & .03 & .03 & .01 & .03 & .06 & .05 & .03 & .09 & .22 & .10 & .06 \\
\hline 28 & .04 & .03 & .03 & .01 & .03 & .06 & .05 & .03 & 3.0 & .22 & .09 & .06 \\
\hline 29 & .04 & .03 & .03 & .01 & ----- & .06 & .05 & .03 & .05 & .20 & .08 & .06 \\
\hline 30 & .04 & .03 & .03 & .01 & ----- & .06 & .05 & .03 & .06 & .19 & .08 & .06 \\
\hline 31 & .04 & ------ & .03 & .01 & ----- & .06 & ----- & .03 & ----- & .19 & .08 & ----- \\
\hline Total & 1.11 & 0.97 & 0.89 & 0.70 & 0.64 & 1.45 & 1.46 & 1.12 & 4.28 & 2.94 & 5.84 & 1.86 \\
\hline Mean & .036 & .032 & .029 & .023 & .022 & .047 & .049 & .036 & .14 & .095 & .19 & .062 \\
\hline Max & .04 & .04 & .03 & .03 & .04 & .07 & .06 & .05 & 3.0 & .29 & .33 & .08 \\
\hline Min & .03 & .02 & .02 & .01 & .01 & .04 & .04 & .03 & .03 & .04 & .08 & .05 \\
\hline $\mathrm{Ac}-\mathrm{Ft}$ & 2.2 & 2.0 & 1.8 & 1.4 & 1.3 & 2.8 & 2.8 & 2.2 & 8.5 & 5.8 & 12 & 3.4 \\
\hline Wtr Year & 2000 & Total & 23. & & lean & .064 & Max & 3.0 & Min & .01 & $\mathrm{Ac}-\mathrm{Ft}$ & 46 \\
\hline Cal Year & 1999 & Total & 23. & & lean & .080 & Max & .77 & Min & .01 & $\mathrm{Ac}-\mathrm{Ft}$ & 46 \\
\hline
\end{tabular}




\section{E245 Pajarito Canyon above TA-18 near Los Alamos, NM}

Location. Lat 3551'4.2", long 106²'11.4", Ramon Vigil Grant, Los Alamos County, 1.5 mi upstream from LANL TA-18 and Three-Mile Canyon and $0.15 \mathrm{mi}$ SE of Pajarito Road.

Drainage Area. $7.84 \mathrm{mi}^{2}$.

Period of Record. November 1993 to September 30, 2000.

Gage. Data logger with cellular telemetry and concrete control. Elevation of gage is $6,880 \mathrm{ft}$. above National Geodetic Vertical Datum of 1929, from GPS survey.

Remarks. Water discharge records fair.

Extremes for Period of Record. Maximum discharge $517 \mathrm{ft}^{3} / \mathrm{s}$, June 28, 2000, gage height $5.03 \mathrm{ft}$ (from flood mark). No flow most of the time.

Extremes for Current Water Year. Maximum discharge $517 \mathrm{ft}^{3} / \mathrm{s}, 1320 \mathrm{hrs}$, June 28, gage height $5.03 \mathrm{ft}$ (from flood mark). No flow most of the time.

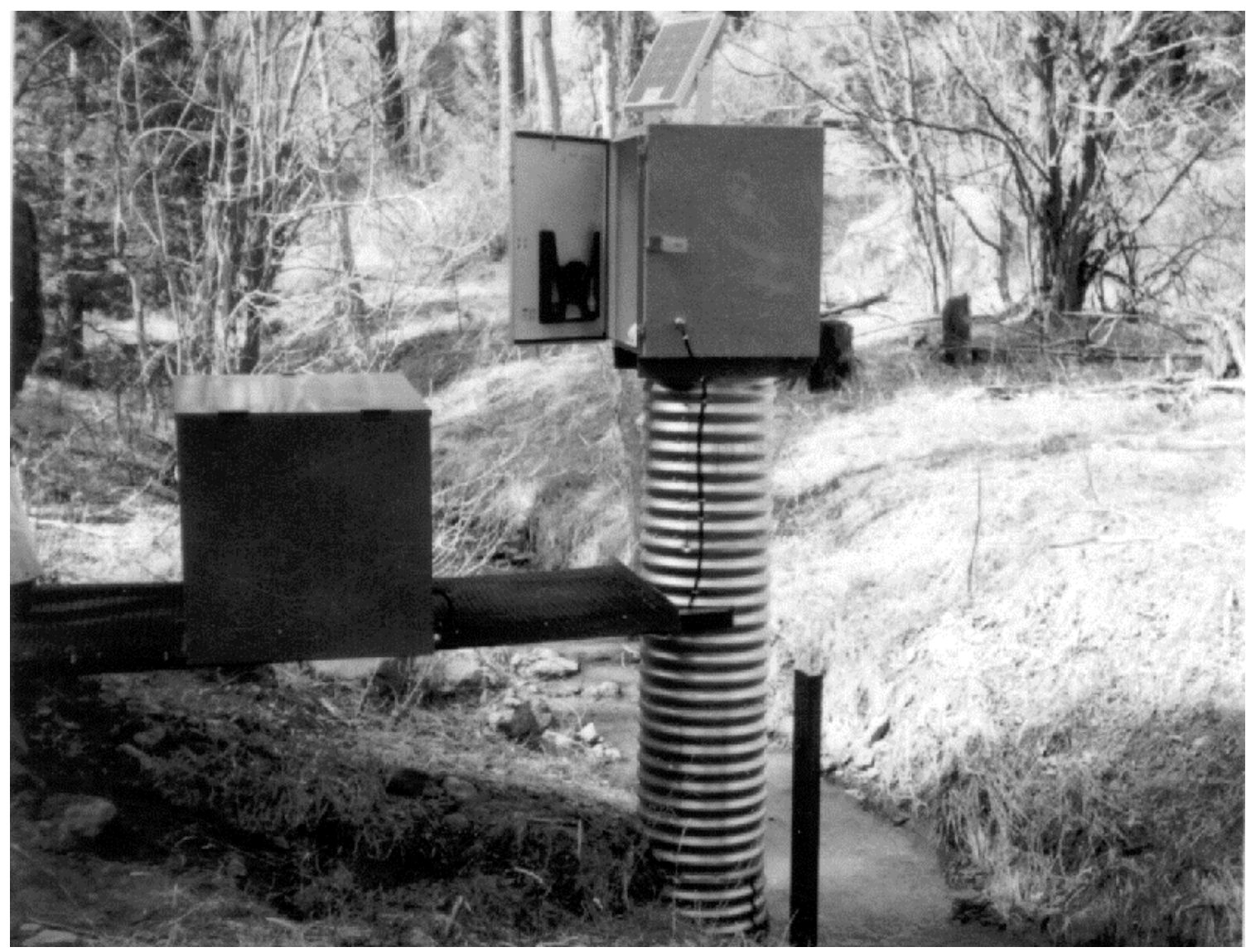


E245 Pajarito Canyon above TA-18 near Los Alamos, NM

Daily Mean Discharge in Cubic Feet per Second

Water Year October 1999 to September 2000

\begin{tabular}{|c|c|c|c|c|c|c|c|c|c|c|c|c|}
\hline DAY & OCT & NOV & DEC & JAN & FEB & MAR & APR & MAY & JUN & JUL & AUG & SEP \\
\hline 1 & 0 & 0 & 0 & 0 & 0 & 0 & 0 & 0 & 0 & 0 & 0 & 0 \\
\hline 2 & 0 & 0 & 0 & 0 & 0 & 0 & 0 & 0 & 0 & 0 & 0 & 0 \\
\hline 3 & 0 & 0 & 0 & 0 & 0 & 0 & 0 & 0 & 0 & 0 & 0 & 0 \\
\hline 4 & 0 & 0 & 0 & 0 & 0 & 0 & 0 & 0 & 0 & 0 & 0 & 0 \\
\hline 5 & 0 & 0 & 0 & 0 & 0 & 0 & 0 & 0 & 0 & 0 & 0 & 0 \\
\hline 6 & 0 & 0 & 0 & 0 & 0 & 0 & 0 & 0 & 0 & 0 & 0 & 0 \\
\hline 7 & 0 & 0 & 0 & 0 & 0 & 0 & 0 & 0 & 0 & 0 & 0 & 0 \\
\hline 8 & 0 & 0 & 0 & 0 & 0 & 0 & 0 & 0 & 0 & 0 & 0 & 0 \\
\hline 9 & 0 & 0 & 0 & 0 & 0 & 0 & 0 & 0 & 0 & 0 & 0 & 0 \\
\hline 10 & 0 & 0 & 0 & 0 & 0 & 0 & 0 & 0 & 0 & 0 & 0 & 0 \\
\hline 11 & 0 & 0 & 0 & 0 & 0 & 0 & 0 & 0 & 0 & .01 & 0 & 0 \\
\hline 12 & 0 & 0 & 0 & 0 & 0 & 0 & 0 & 0 & 0 & .01 & 0 & 0 \\
\hline 13 & 0 & 0 & 0 & 0 & 0 & 0 & 0 & 0 & 0 & 0 & 0 & 0 \\
\hline 14 & 0 & 0 & 0 & 0 & 0 & 0 & 0 & 0 & 0 & .02 & 0 & 0 \\
\hline 15 & 0 & 0 & 0 & 0 & 0 & 0 & 0 & 0 & 0 & .06 & 0 & 0 \\
\hline 16 & 0 & 0 & 0 & 0 & 0 & 0 & 0 & 0 & 0 & 0 & 0 & 0 \\
\hline 17 & 0 & 0 & 0 & 0 & 0 & 0 & 0 & 0 & 0 & 0 & 0 & 0 \\
\hline 18 & 0 & 0 & 0 & 0 & 0 & 0 & 0 & 0 & 0 & 0 & 0 & 0 \\
\hline 19 & 0 & 0 & 0 & 0 & 0 & 0 & 0 & 0 & 0 & 0 & 0 & 0 \\
\hline 20 & 0 & 0 & 0 & 0 & 0 & 0 & 0 & 0 & 0 & 0 & 0 & 0 \\
\hline 21 & 0 & 0 & 0 & 0 & 0 & 0 & 0 & 0 & 0 & 0 & 0 & 0 \\
\hline 22 & 0 & 0 & 0 & 0 & 0 & 0 & 0 & 0 & 0 & 0 & 0 & 0 \\
\hline 23 & 0 & 0 & 0 & 0 & 0 & 0 & 0 & 0 & 0 & 0 & 0 & 0 \\
\hline 24 & 0 & 0 & 0 & 0 & 0 & 0 & 0 & 0 & 0 & 0 & 0 & 0 \\
\hline 25 & 0 & 0 & 0 & 0 & 0 & 0 & 0 & 0 & 0 & 0 & 0 & 0 \\
\hline 26 & 0 & 0 & 0 & 0 & 0 & 0 & 0 & 0 & 0 & 0 & 0 & 0 \\
\hline 27 & 0 & 0 & 0 & 0 & 0 & 0 & 0 & 0 & 0 & 0 & 0 & 0 \\
\hline 28 & 0 & 0 & 0 & 0 & 0 & 0 & 0 & 0 & 5.4 & 0 & 0 & 0 \\
\hline 29 & 0 & 0 & 0 & 0 & ------ & 0 & 0 & 0 & .02 & .15 & 0 & 0 \\
\hline 30 & 0 & 0 & 0 & 0 & ------ & 0 & 0 & 0 & .11 & 0 & 0 & 0 \\
\hline 31 & 0 & ----- & 0 & 0 & ----- & 0 & ----- & 0 & ----- & 0 & 0 & ---- \\
\hline Total & 0 & 0 & 0 & 0 & 0 & 0 & 0 & 0 & 5.53 & .025 & 0 & 0 \\
\hline Mean & 0 & 0 & 0 & 0 & 0 & 0 & 0 & 0 & .18 & .008 & 0 & 0 \\
\hline Max & 0 & 0 & 0 & 0 & 0 & 0 & 0 & 0 & 5.4 & .15 & 0 & 0 \\
\hline Min & 0 & 0 & 0 & 0 & 0 & 0 & 0 & 0 & 0 & 0 & 0 & 0 \\
\hline $\mathrm{Ac}-\mathrm{Ft}$ & 0 & 0 & 0 & 0 & 0 & 0 & 0 & 0 & 11 & .5 & 0 & 0 \\
\hline Wtr Year & 2000 & Total & & & Mean & .016 & Max & 5.4 & Min & 0 & $\mathrm{Ac}-\mathrm{Ft}$ & 12 \\
\hline Cal Year & 1999 & Total & & & Mean & .020 & Max & .62 & Min & 0 & $\mathrm{Ac}-\mathrm{Ft}$ & 12 \\
\hline
\end{tabular}




\section{E250 Pajarito Canyon above Highway 4 near White Rock, NM}

Location. Lat $35^{\circ} 49^{\prime} 26.7^{\prime \prime}$, long $106^{\circ} 13^{\prime} 40.5^{\prime \prime}$, in Ramon Vigil Grant, Los Alamos County, 0.25 mi upstream from NM State Highway 4 and White Rock, NM.

Drainage Area. $10.9 \mathrm{mi}^{2}$.

Period of Record. November 1993 to September 30, 2000.

Gage. Data logger with cellular telemetry and concrete control. Elevation of gage is 6,535 ft above National Geodetic Vertical Datum of 1929, from GPS survey.

Remarks. Water discharge records good.

Extremes for Period of Record. Maximum discharge $20 \mathrm{ft}^{3} / \mathrm{s}$, June 17, 1999, gage height $3.71 \mathrm{ft}$. No flow most of the time.

Extremes for Current Water Year. Maximum discharge $14 \mathrm{ft}^{3} / \mathrm{s}$ at $1710 \mathrm{hrs}$, June 28, gage height $3.44 \mathrm{ft}$. No flow most of time.

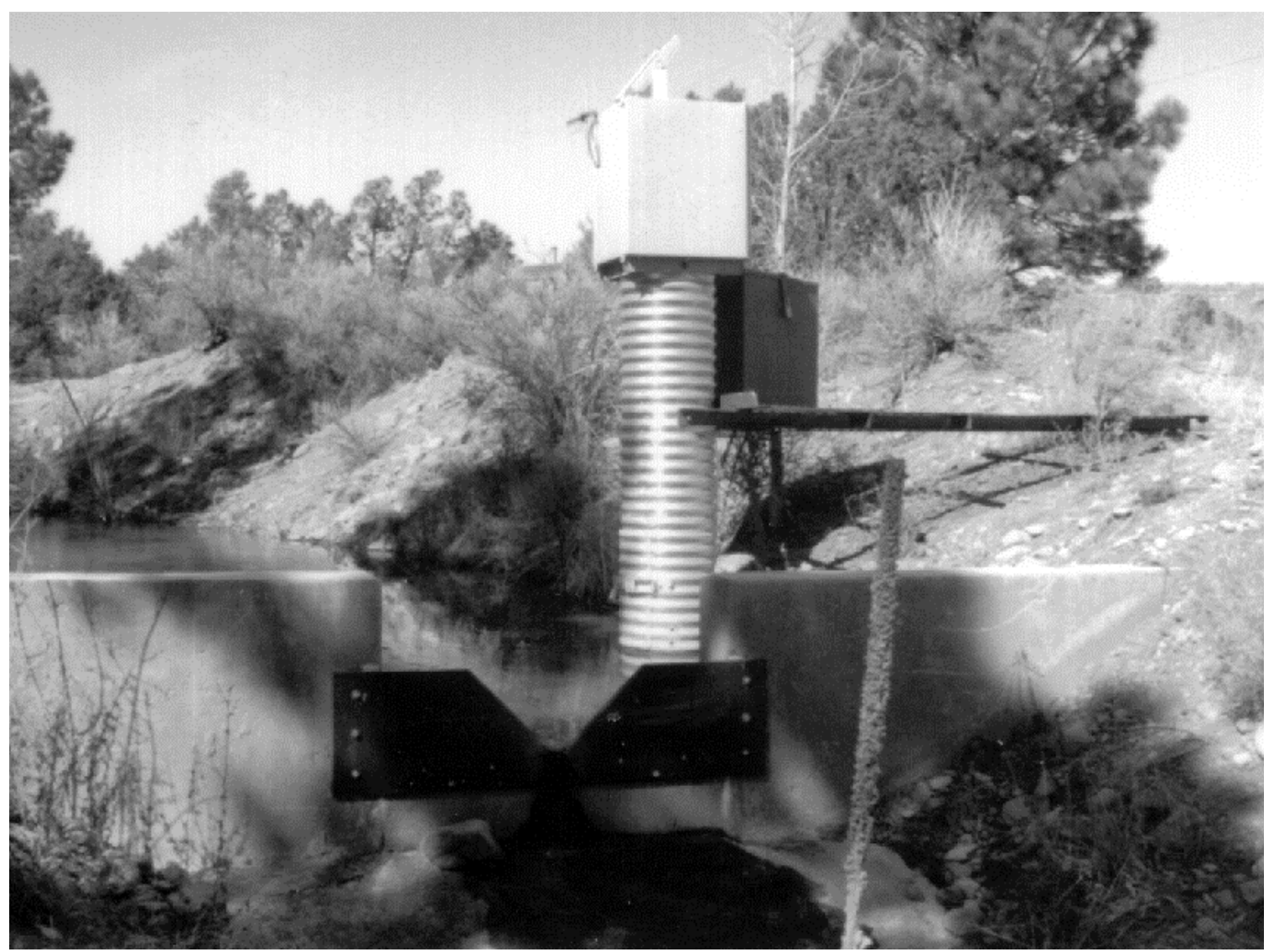




\section{E250 Pajarito Canyon above Highway 4 near White Rock, NM}

Daily Mean Discharge in Cubic Feet per Second

Water Year October 1999 to September 2000

\begin{tabular}{|c|c|c|c|c|c|c|c|c|c|c|c|c|}
\hline DAY & OCT & NOV & DEC & JAN & FEB & MAR & APR & MAY & JUN & JUL & AUG & SEP \\
\hline 1 & 0 & 0 & 0 & 0 & 0 & 0 & 0 & 0 & 0 & 0 & 0 & 0 \\
\hline 2 & 0 & 0 & 0 & 0 & 0 & 0 & 0 & 0 & 0 & 0 & 0 & 0 \\
\hline 3 & 0 & 0 & 0 & 0 & 0 & 0 & 0 & 0 & 0 & 0 & 0 & 0 \\
\hline 4 & 0 & 0 & 0 & 0 & 0 & 0 & 0 & 0 & 0 & 0 & 0 & 0 \\
\hline 5 & 0 & 0 & 0 & 0 & 0 & 0 & 0 & 0 & 0 & 0 & 0 & 0 \\
\hline 6 & 0 & 0 & 0 & 0 & 0 & 0 & 0 & 0 & 0 & 0 & 0 & 0 \\
\hline 7 & 0 & 0 & 0 & 0 & 0 & 0 & 0 & 0 & 0 & 0 & 0 & 0 \\
\hline 8 & 0 & 0 & 0 & 0 & 0 & 0 & 0 & 0 & 0 & 0 & 0 & 0 \\
\hline 9 & 0 & 0 & 0 & 0 & 0 & 0 & 0 & 0 & 0 & 0 & 0 & 0 \\
\hline 10 & 0 & 0 & 0 & 0 & 0 & 0 & 0 & 0 & 0 & 0 & 0 & 0 \\
\hline 11 & 0 & 0 & 0 & 0 & 0 & 0 & 0 & 0 & 0 & 0 & 0 & 0 \\
\hline 12 & 0 & 0 & 0 & 0 & 0 & 0 & 0 & 0 & 0 & 0 & 0 & 0 \\
\hline 13 & 0 & 0 & 0 & 0 & 0 & 0 & 0 & 0 & 0 & 0 & 0 & 0 \\
\hline 14 & 0 & 0 & 0 & 0 & 0 & 0 & 0 & 0 & 0 & 0 & 0 & 0 \\
\hline 15 & 0 & 0 & 0 & 0 & 0 & 0 & 0 & 0 & 0 & 0 & 0 & 0 \\
\hline 16 & 0 & 0 & 0 & 0 & 0 & 0 & 0 & 0 & 0 & 0 & 0 & 0 \\
\hline 17 & 0 & 0 & 0 & 0 & 0 & 0 & 0 & 0 & 0 & 0 & 0 & 0 \\
\hline 18 & 0 & 0 & 0 & 0 & 0 & 0 & 0 & 0 & 0 & 0 & 0 & 0 \\
\hline 19 & 0 & 0 & 0 & 0 & 0 & 0 & 0 & 0 & 0 & 0 & 0 & 0 \\
\hline 20 & 0 & 0 & 0 & 0 & 0 & 0 & 0 & 0 & 0 & 0 & 0 & 0 \\
\hline 21 & 0 & 0 & 0 & 0 & 0 & 0 & 0 & 0 & 0 & 0 & 0 & 0 \\
\hline 22 & 0 & 0 & 0 & 0 & 0 & 0 & 0 & 0 & 0 & 0 & 0 & 0 \\
\hline 23 & 0 & 0 & 0 & 0 & 0 & 0 & 0 & 0 & 0 & 0 & 0 & 0 \\
\hline 24 & 0 & 0 & 0 & 0 & 0 & 0 & 0 & 0 & 0 & 0 & 0 & 0 \\
\hline 25 & 0 & 0 & 0 & 0 & 0 & 0 & 0 & 0 & 0 & 0 & 0 & 0 \\
\hline 26 & 0 & 0 & 0 & 0 & 0 & 0 & 0 & 0 & 0 & 0 & 0 & 0 \\
\hline 27 & 0 & 0 & 0 & 0 & 0 & 0 & 0 & 0 & 0 & 0 & 0 & 0 \\
\hline 28 & 0 & 0 & 0 & 0 & 0 & 0 & 0 & 0 & 1.4 & 0 & 0 & 0 \\
\hline 29 & 0 & 0 & 0 & 0 & ----- & 0 & 0 & 0 & .09 & 0 & 0 & 0 \\
\hline 30 & 0 & 0 & 0 & 0 & ----- & 0 & 0 & 0 & 0 & 0 & 0 & 0 \\
\hline 31 & 0 & ----- & 0 & 0 & ------ & 0 & ------ & 0 & ----- & 0 & 0 & ----- \\
\hline Total & 0 & 0 & 0 & 0 & 0 & 0 & 0 & 0 & 1.49 & 0 & 0 & 0 \\
\hline Mean & 0 & 0 & 0 & 0 & 0 & 0 & 0 & 0 & .050 & 0 & 0 & 0 \\
\hline Max & 0 & 0 & 0 & 0 & 0 & 0 & 0 & 0 & 1.4 & 0 & 0 & 0 \\
\hline Min & 0 & 0 & 0 & 0 & 0 & 0 & 0 & 0 & 0 & 0 & 0 & 0 \\
\hline $\mathrm{Ac}-\mathrm{Ft}$ & 0 & 0 & 0 & 0 & 0 & 0 & 0 & 0 & 3.0 & 0 & 0 & 0 \\
\hline Wtr Year & 2000 & Total & & & Mean & .004 & Max & 1.4 & Min & 0 & Ac-Ft & 3.0 \\
\hline Cal Year & 1999 & Total & & & Mean & .005 & Max & 1.5 & Min & 0 & $\mathrm{Ac}-\mathrm{Ft}$ & 3.3 \\
\hline
\end{tabular}




\section{E252 Water Canyon above Highway 501 near Los Alamos, NM}

Location. Lat 3550'18", long 106²1'42.6", T. 19 N, R. 5 E., Los Alamos County in Santa Fe National Forest, $0.3 \mathrm{mi}$ upstream from NM State Highway 501 and $0.3 \mathrm{mi} \mathrm{NW}$ of junction of State Highways 501 and 4.

Drainage Area. $3.39 \mathrm{mi}^{2}$.

Period of Record. October 1994 to September 30, 2000.

Gage. Data logger with cellular telemetry and $120^{\circ}$ weir plate. Elevation of gage is 7,558 $\mathrm{ft}$ above National Geodetic Vertical Datum of 1929, from GPS survey.

Remarks. Records good until June 28, when gage was disabled by flood.

Extremes for Period of Record. Maximum discharge $840 \mathrm{ft}^{3} / \mathrm{s}$ on June 28, 2000, from peak flow computation, gage height $7.91 \mathrm{ft}$. No flow at times.

Extremes for Current Water Year. Maximum discharge $840 \mathrm{ft}^{3} / \mathrm{s}$ at about $1230 \mathrm{hrs,} \mathrm{June} \mathrm{28,}$ from peak flow computation, gage height $7.91 \mathrm{ft}$. No flow at times.

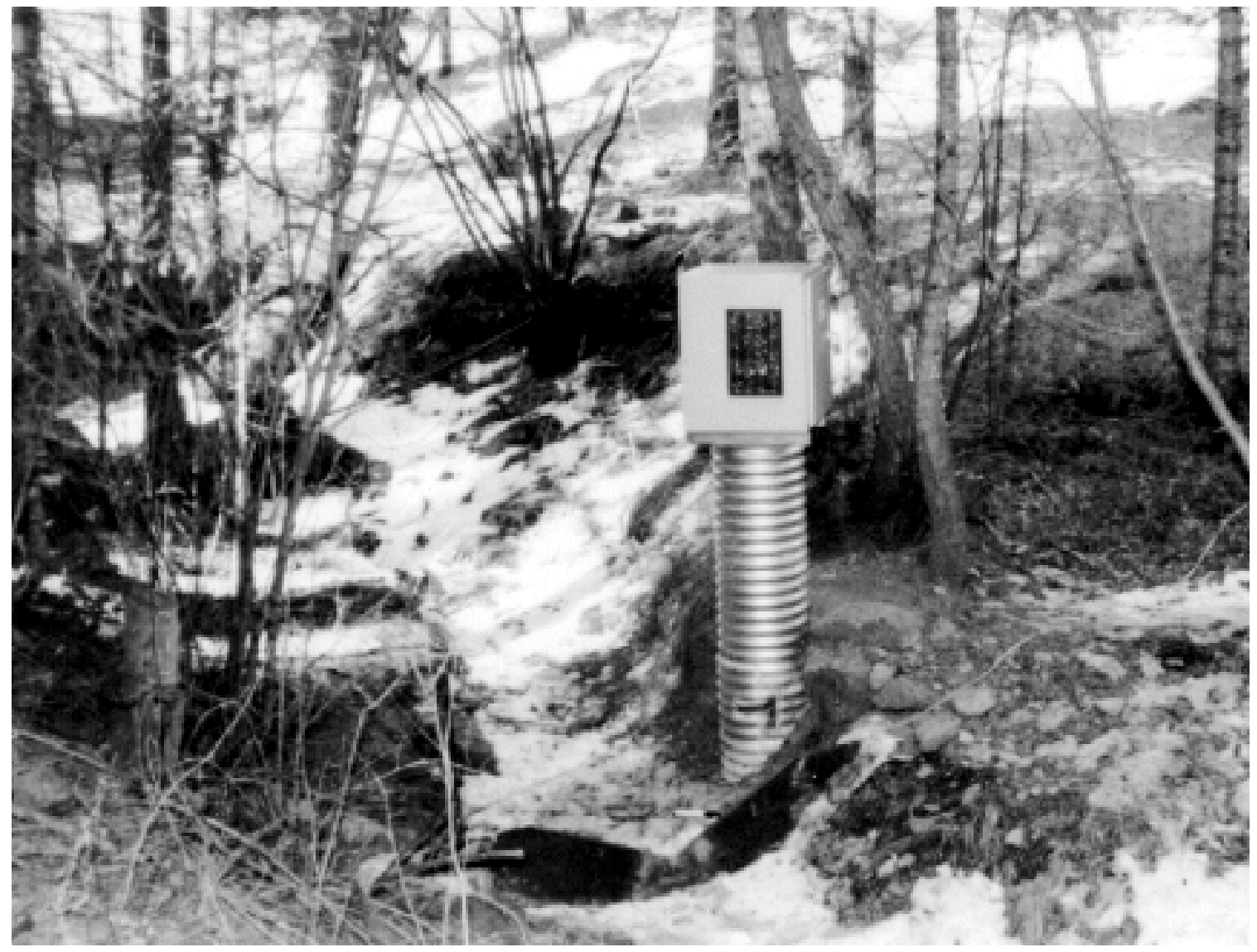




\section{E252 Water Canyon above Highway 501 near Los Alamos, NM}

Daily Mean Discharge in Cubic Feet per Second

Water Year October 1999 to September 2000

\begin{tabular}{|c|c|c|c|c|c|c|c|c|c|c|c|c|}
\hline DAY & OCT & NOV & DEC & JAN & FEB & MAR & APR & MAY & JUN & JUL & AUG & SEP \\
\hline 1 & .02 & .01 & .01 & .01 & .02 & .02 & .02 & .04 & .02 & & & \\
\hline 2 & .02 & .01 & .01 & .01 & .02 & .02 & .02 & .05 & .02 & & & \\
\hline 3 & .02 & .01 & .01 & .01 & .02 & .02 & .02 & .03 & .02 & & & \\
\hline 4 & .02 & .01 & .01 & .01 & .02 & .02 & .02 & .03 & .02 & & & \\
\hline 5 & .02 & .01 & .01 & .01 & .02 & .02 & .02 & .03 & .01 & & & \\
\hline 6 & .02 & .01 & .01 & .01 & .02 & .02 & .02 & .03 & .02 & & & \\
\hline 7 & .02 & .01 & .01 & .01 & .02 & .02 & .02 & .02 & .02 & & & \\
\hline 8 & .02 & .01 & .01 & .01 & .02 & .02 & .02 & .02 & .02 & & & \\
\hline 9 & .02 & .01 & .01 & .01 & .02 & .02 & .02 & .02 & .02 & & & \\
\hline 10 & .02 & .01 & .01 & .01 & .02 & .02 & .02 & .02 & .02 & & & \\
\hline 11 & .02 & .01 & .01 & .01 & .02 & .02 & .02 & .03 & .02 & & & \\
\hline 12 & .02 & .01 & .01 & .01 & .02 & .02 & .02 & .03 & .01 & & & \\
\hline 13 & .02 & .01 & .01 & .01 & .02 & .02 & .02 & .02 & .02 & & & \\
\hline 14 & .02 & .01 & .01 & .01 & .02 & .02 & .03 & .02 & .02 & & & \\
\hline 15 & .02 & .01 & .01 & .01 & .02 & .02 & .03 & .02 & .02 & & & \\
\hline 16 & .02 & .01 & .01 & .01 & .02 & .02 & .03 & .02 & .02 & & & \\
\hline 17 & .01 & .01 & .01 & .01 & .02 & .02 & .04 & .02 & .02 & & & \\
\hline 18 & .01 & .01 & .01 & .01 & .02 & .02 & .04 & .02 & .02 & & & \\
\hline 19 & .01 & .01 & .01 & .01 & .02 & .02 & .04 & .02 & .01 & & & \\
\hline 20 & .01 & .01 & .01 & .01 & .02 & .02 & .03 & .02 & .01 & & & \\
\hline 21 & .01 & .01 & .01 & .01 & .02 & .02 & .04 & .02 & .01 & & & \\
\hline 22 & .01 & .01 & .01 & .01 & .02 & .02 & .04 & .02 & .02 & & & \\
\hline 23 & .01 & .01 & .01 & .01 & .02 & .02 & .04 & .02 & .02 & & & \\
\hline 24 & .01 & .01 & .01 & .02 & .02 & .02 & .05 & .02 & .02 & & & \\
\hline 25 & .01 & .01 & .01 & .02 & .02 & .02 & .05 & .02 & .02 & & & \\
\hline 26 & .01 & .01 & .01 & .02 & .02 & .03 & .05 & .02 & .02 & & & \\
\hline 27 & .01 & .01 & .01 & .02 & .02 & .03 & .05 & .02 & .02 & & & \\
\hline 28 & .01 & .01 & .01 & .02 & .02 & .02 & .05 & .02 & 28 & & & \\
\hline 29 & .02 & .01 & .01 & .02 & ----- & .02 & .04 & .02 & .05 & & & \\
\hline 30 & .01 & .01 & .01 & .02 & ------ & .02 & .04 & .02 & .03 & & & \\
\hline 31 & .01 & ----- & .01 & .02 & ----- & .02 & ------ & .02 & ----- & & & \\
\hline Total & 0.48 & 0.30 & 0.31 & 0.39 & 0.58 & 0.64 & 0.95 & 0.73 & 28.57 & & & \\
\hline Mean & .015 & .010 & .010 & .013 & .020 & .021 & .032 & .024 & .95 & & & \\
\hline Max & .02 & .01 & .01 & .02 & .02 & .03 & .05 & .05 & 28 & & & \\
\hline Min & .01 & .01 & .01 & .01 & .02 & .02 & .02 & .02 & .01 & & & \\
\hline$A c-F t$ & 1 & .6 & .6 & .8 & .9 & 1.1 & 1.9 & 1.5 & 57 & & & \\
\hline Wtr Year & 2000 & Total & 32.9 & & ean & .12 & Max & 28 & Min & .01 & $\mathrm{Ac}-\mathrm{Ft}$ & 66 \\
\hline Cal Year & 1999 & Total & 5.0 & & ean & .014 & Max & .05 & Min & .01 & $\mathrm{Ac}-\mathrm{Ft}$ & 10 \\
\hline
\end{tabular}




\section{E253 Cañon del Valle above Highway 501 near Los Alamos, NM}

Location. Lat 3551'6.6", long 106²1'17", NE 1/4, NE 1/4, sec. 25, T. 19 N, R. 5 E, Los Alamos County in Santa Fe National Forest, 0.25 mi upstream from NM State Highway 501, $4.7 \mathrm{mi}$ above mouth and $1.5 \mathrm{mi} \mathrm{N}$ of junction of State Highways 501 and 4.

Drainage Area. $2.46 \mathrm{mi}^{2}$.

Period of Record. October 1994 to September 30, 2000.

Gage. Data logger with cellular telemetry and $120^{\circ}$ weir plate. Elevation of gage is $7,707 \mathrm{ft}$ above National Geodetic Vertical Datum of 1929, from GPS survey.

Remarks. Records good until June 28, when gage was destroyed by flood.

Extremes for Period of Record. Maximum dischage $740 \mathrm{ft}^{3} / \mathrm{s}$, June 28, 2000, from peak flow computation, gage height $8.42 \mathrm{ft}$. No flow most of time.

Extremes for Current Water Year. Maximum discharge $740 \mathrm{ft}^{3} / \mathrm{s}$ at about $1230 \mathrm{hrs,} \mathrm{June} \mathrm{28,}$ from peak flow computation, gage height $8.42 \mathrm{ft}$. No flow most of time.

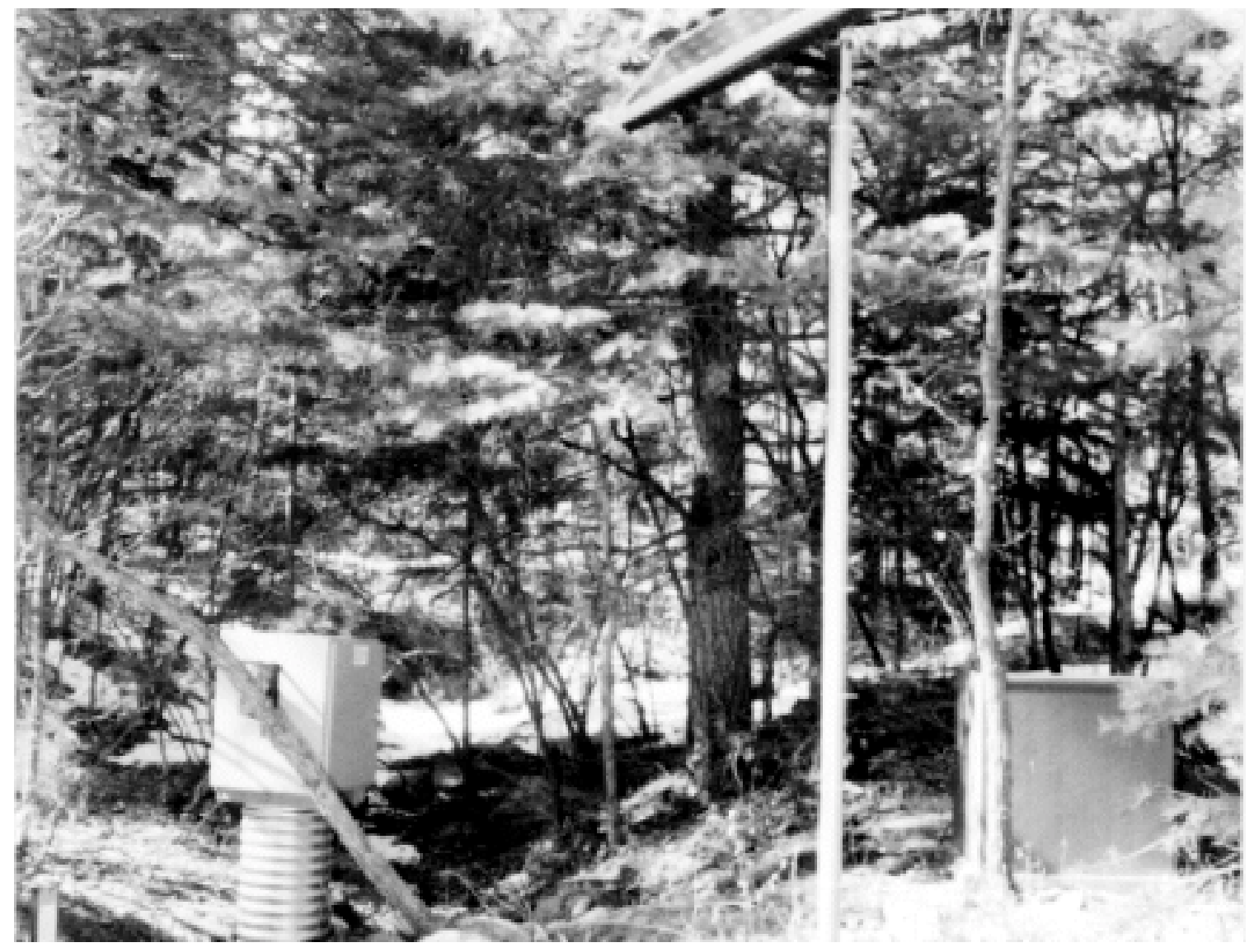




\section{E253 Cañon del Valle above Highway 501 near Los Alamos, NM}

Daily Mean Discharge in Cubic Feet per Second

Water Year October 1999 to September 2000

\begin{tabular}{|c|c|c|c|c|c|c|c|c|c|c|c|c|}
\hline DAY & ОСт & NOV & DEC & JAN & FEB & MAR & APR & MAY & JUN & JUL & AUG & SEP \\
\hline 1 & $0^{*}$ & $0^{*}$ & 0 & 0 & 0 & 0 & 0 & 0 & $0^{*}$ & & & \\
\hline 2 & 0 & $0^{*}$ & 0 & 0 & 0 & 0 & 0 & 0 & $0^{*}$ & & & \\
\hline 3 & 0 & $0^{*}$ & 0 & 0 & 0 & 0 & 0 & $0^{*}$ & $0^{*}$ & & & \\
\hline 4 & 0 & $0^{*}$ & 0 & 0 & 0 & 0 & 0 & $0^{*}$ & $0^{*}$ & & & \\
\hline 5 & 0 & 0 & 0 & 0 & 0 & 0 & 0 & $0^{*}$ & $0^{*}$ & & & \\
\hline 6 & $0^{*}$ & 0 & 0 & 0 & 0 & 0 & 0 & $0^{*}$ & $0^{*}$ & & & \\
\hline 7 & $0^{*}$ & 0 & 0 & 0 & 0 & 0 & 0 & $0^{*}$ & $0^{*}$ & & & \\
\hline 8 & $0^{*}$ & 0 & 0 & 0 & 0 & 0 & 0 & $0^{*}$ & $0^{*}$ & & & \\
\hline 9 & $0^{*}$ & 0 & 0 & 0 & 0 & 0 & 0 & $0^{*}$ & $0^{*}$ & & & \\
\hline 10 & $0^{*}$ & 0 & 0 & 0 & 0 & 0 & 0 & $0^{*}$ & $0^{*}$ & & & \\
\hline 11 & $0^{*}$ & 0 & 0 & 0 & 0 & 0 & 0 & $0^{*}$ & $0^{*}$ & & & \\
\hline 12 & $0^{*}$ & 0 & 0 & 0 & 0 & 0 & 0 & $0^{*}$ & $0^{*}$ & & & \\
\hline 13 & $0^{*}$ & 0 & 0 & 0 & 0 & 0 & 0 & $0^{*}$ & $0^{*}$ & & & \\
\hline 14 & $0^{*}$ & 0 & 0 & 0 & 0 & 0 & 0 & $0^{*}$ & $0^{*}$ & & & \\
\hline 15 & $0^{*}$ & 0 & 0 & 0 & 0 & 0 & 0 & $0^{*}$ & $0^{*}$ & & & \\
\hline 16 & $0^{*}$ & 0 & 0 & 0 & 0 & 0 & 0 & $0^{*}$ & $0^{*}$ & & & \\
\hline 17 & $0^{*}$ & 0 & 0 & 0 & 0 & 0 & 0 & $0^{*}$ & $0^{*}$ & & & \\
\hline 18 & $0^{*}$ & 0 & 0 & 0 & 0 & 0 & 0 & $0^{*}$ & $0^{*}$ & & & \\
\hline 19 & $0^{*}$ & 0 & 0 & 0 & 0 & 0 & 0 & $0^{*}$ & $0^{*}$ & & & \\
\hline 20 & $0^{*}$ & 0 & 0 & 0 & 0 & 0 & 0 & $0^{*}$ & $0^{*}$ & & & \\
\hline 21 & $0^{*}$ & 0 & 0 & 0 & 0 & 0 & 0 & $0^{*}$ & $0^{*}$ & & & \\
\hline 22 & $0^{*}$ & 0 & 0 & 0 & 0 & 0 & 0 & $0^{*}$ & $0^{*}$ & & & \\
\hline 23 & $0^{*}$ & 0 & 0 & 0 & 0 & 0 & 0 & $0^{*}$ & $0^{*}$ & & & \\
\hline 24 & $0^{*}$ & 0 & 0 & 0 & 0 & 0 & 0 & $0^{*}$ & $0^{*}$ & & & \\
\hline 25 & $0^{*}$ & 0 & 0 & 0 & 0 & 0 & 0 & $0^{*}$ & $0^{*}$ & & & \\
\hline 26 & $0^{*}$ & 0 & 0 & 0 & 0 & 0 & 0 & $0^{*}$ & $0^{*}$ & & & \\
\hline 27 & $0^{*}$ & 0 & 0 & 0 & 0 & 0 & 0 & $0^{*}$ & $0^{*}$ & & & \\
\hline 28 & $0^{*}$ & 0 & 0 & 0 & 0 & 0 & 0 & $0^{*}$ & 26 & & & \\
\hline 29 & $0^{*}$ & 0 & 0 & 0 & ----- & 0 & 0 & $0^{*}$ & $0^{*}$ & & & \\
\hline 30 & $0^{*}$ & 0 & 0 & 0 & ------ & 0 & 0 & $0^{*}$ & $0^{*}$ & & & \\
\hline 31 & $0^{*}$ & ------ & 0 & 0 & ----- & 0 & ----- & $0^{*}$ & ----- & & & \\
\hline Total & 0 & 0 & 0 & 0 & 0 & 0 & 0 & 0 & 26 & & & \\
\hline Mean & 0 & 0 & 0 & 0 & 0 & 0 & 0 & 0 & .87 & & & \\
\hline Max & 0 & 0 & 0 & 0 & 0 & 0 & 0 & 0 & 26 & & & \\
\hline Min & 0 & 0 & 0 & 0 & 0 & 0 & 0 & 0 & 0 & & & \\
\hline $\mathrm{Ac}-\mathrm{Ft}$ & 0 & 0 & 0 & 0 & 0 & 0 & 0 & 0 & 52 & & & \\
\hline Wtr Year & 2000 & Total & 26 & & lean & .095 & $\operatorname{Max}$ & 26 & Min & 0 & $\mathrm{Ac}-\mathrm{Ft}$ & 52 \\
\hline Cal Year & 1999 & Total & 0 & & lean & 0 & $\operatorname{Max}$ & 0 & Min & 0 & Ac- $\mathrm{Ft}$ & 0 \\
\hline
\end{tabular}

${ }^{\star}$ Estimated. 


\section{E263 Water Canyon at Highway 4 near White Rock, NM}

Location. Lat 3548'20.0", long 106 $14^{\circ} 52.0^{\prime \prime}$ in Ramon Vigil Grant, Los Alamos County, 4.0 mi SW of White Rock and $50 \mathrm{ft}$ downstream from NM State Highway 4 and $150 \mathrm{ft}$ above mouth of Indio Canyon.

Drainage Area. $8.20 \mathrm{mi}^{2}$.

Period of Record. April 1999 to September 30, 2000.

Gage. Data logger with cellular telemetry. Elevation of gage is 6,368 ft above National Geodetic Vertical Datum of 1929, from GPS survey.

Remarks. Water discharge records good.

Extremes for Period of Record. Maximum discharge $306 \mathrm{ft}^{3} / \mathrm{s}$, June 28, 2000, gage height $3.78 \mathrm{ft}$. No flow most of time.

Extremes for May 1999-September 2000. Maximum discharge $306 \mathrm{ft}^{3} / \mathrm{s}$ at $1350 \mathrm{hrs,} \mathrm{June} \mathrm{28,}$ gage height $3.78 \mathrm{ft}$. No flow most of time.

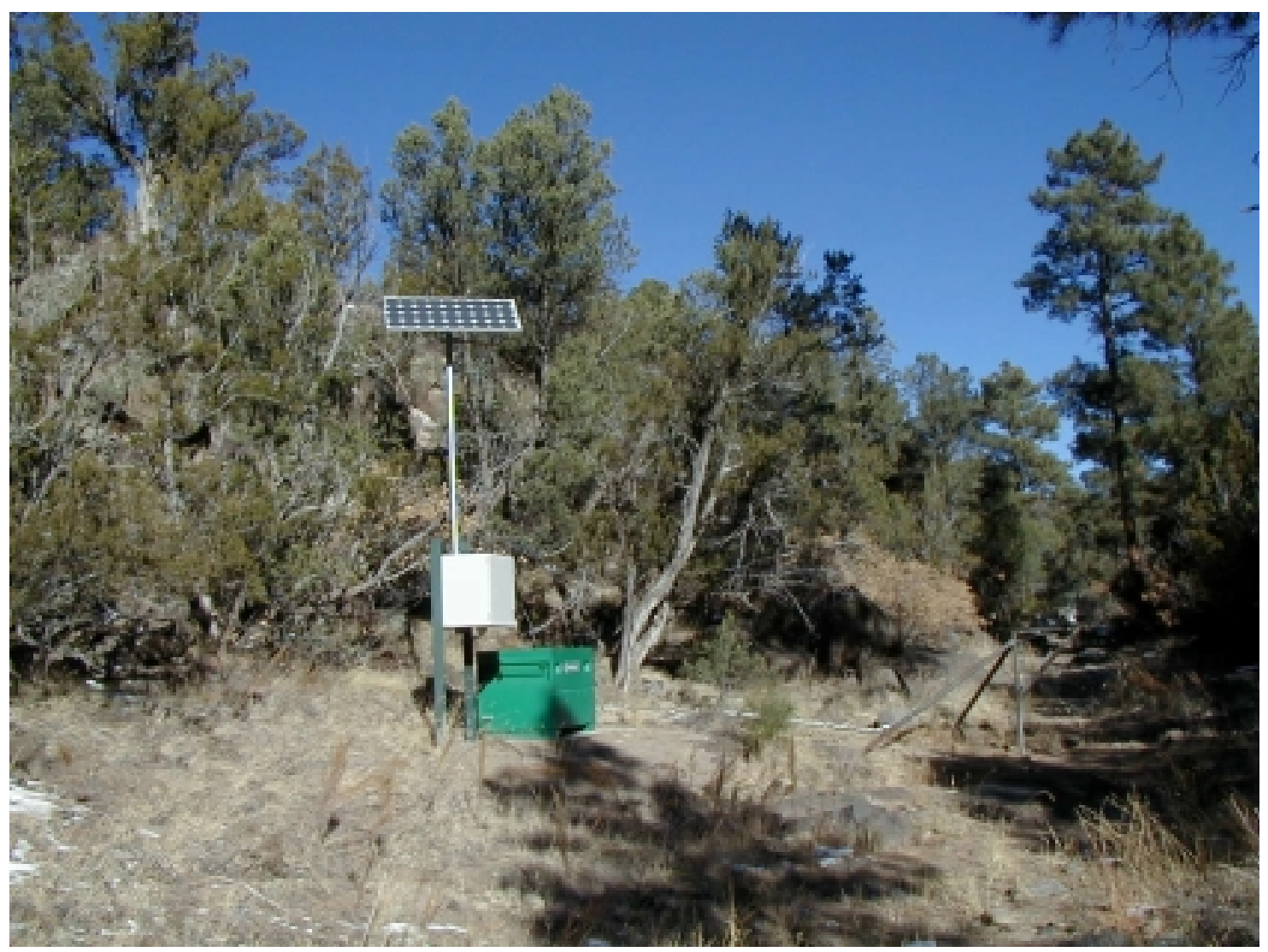




\section{E263 Water Canyon at Highway 4 near White Rock, NM}

Daily Mean Discharge in Cubic Feet per Second

Water Year October 1998 to September 1999

\begin{tabular}{|c|c|c|c|c|c|c|c|c|c|c|c|}
\hline DAY & OCT & NOV & DEC & FEB & MAR & APR & MAY & JUN & JUL & AUG & SEP \\
\hline 1 & & & & & & & 0 & 0 & 0 & 0 & 0 \\
\hline 2 & & & & & & & 0 & 0 & 0 & 0 & 0 \\
\hline 3 & & & & & & & 0 & 0 & 0 & 0 & 0 \\
\hline 4 & & & & & & & 0 & 0 & 0 & 0 & 0 \\
\hline 5 & & & & & & & 0 & 0 & 0 & 0 & 0 \\
\hline 6 & & & & & & & 0 & 0 & 0 & 0 & 0 \\
\hline 7 & & & & & & & 0 & 0 & 0 & 0 & 0 \\
\hline 8 & & & & & & & .03 & 0 & .18 & 0 & 0 \\
\hline 9 & & & & & & & 0 & 0 & .03 & 0 & 0 \\
\hline 10 & & & & & & & 0 & 0 & 0 & 0 & 0 \\
\hline 11 & & & & & & & 0 & 0 & 0 & 0 & 0 \\
\hline 12 & & & & & & & 0 & 0 & 0 & 0 & 0 \\
\hline 13 & & & & & & & 0 & .02 & 0 & 0 & 0 \\
\hline 14 & & & & & & & 0 & 0 & 0 & 0 & 0 \\
\hline 15 & & & & & & & 0 & 0 & 0 & 0 & 0 \\
\hline 16 & & & & & & & 0 & 0 & 0 & 0 & 0 \\
\hline 17 & & & & & & & 0 & .40 & 0 & 0 & 0 \\
\hline 18 & & & & & & & 0 & 0 & 0 & 0 & 0 \\
\hline 19 & & & & & & & 0 & 0 & 0 & 0 & 0 \\
\hline 20 & & & & & & & 0 & 0 & 0 & 0 & 0 \\
\hline 21 & & & & & & & 0 & .11 & 0 & 0 & 0 \\
\hline 22 & & & & & & & 0 & 0 & 0 & 0 & 0 \\
\hline 23 & & & & & & & 0 & 0 & 0 & 0 & 0 \\
\hline 24 & & & & & & & .04 & 0 & 0 & 0 & 0 \\
\hline 25 & & & & & & & 0 & 0 & 0 & 0 & 0 \\
\hline 26 & & & & & & & 0 & 0 & 0 & 0 & 0 \\
\hline 27 & & & & & & & 0 & 0 & 0 & 0 & 0 \\
\hline 28 & & & & & & & 0 & 0 & 0 & 0 & 0 \\
\hline 29 & & & & & & & 0 & 0 & 0 & 0 & 0 \\
\hline 30 & & & & & & & 0 & 0 & 0 & 0 & 0 \\
\hline 31 & & & & & & & 0 & ----- & 0 & 0 & ----- \\
\hline Total & & & & & & & 0.07 & 0.53 & 0.21 & 0 & 0 \\
\hline Mean & & & & & & & .004 & .018 & .007 & 0 & 0 \\
\hline Max & & & & & & & .04 & .40 & .18 & 0 & 0 \\
\hline Min & & & & & & & 0 & 0 & 0 & 0 & 0 \\
\hline Ac-Ft & & & & & & & .14 & 1.0 & .5 & 0 & 0 \\
\hline Wtr Year & 1999 & Total & 0.81 & Mean & .006 & Max & .40 & Min & 0 & $\mathrm{Ac}-\mathrm{Ft}$ & 1.6 \\
\hline Cal Year & 1998 & Total & 0.92 & Mean & .010 & Max & .01 & Min & .01 & $\mathrm{Ac}-\mathrm{Ft}$ & 1.8 \\
\hline
\end{tabular}




\section{E263 Water Canyon at Highway 4 near White Rock, NM}

Daily Mean Discharge in Cubic Feet per Second

Water Year October 1999 to September 2000

\begin{tabular}{|c|c|c|c|c|c|c|c|c|c|c|c|c|}
\hline DAY & OCT & NOV & DEC & JAN & FEB & MAR & APR & MAY & JUN & JUL & AUG & SEP \\
\hline 1 & 0 & 0 & 0 & 0 & 0 & 0 & 0 & 0 & 0 & 0 & 0 & 0 \\
\hline 2 & 0 & 0 & 0 & 0 & 0 & 0 & 0 & 0 & 0 & 0 & 0 & 0 \\
\hline 3 & 0 & 0 & 0 & 0 & 0 & 0 & 0 & 0 & 0 & 0 & 0 & 0 \\
\hline 4 & 0 & 0 & 0 & 0 & 0 & 0 & 0 & 0 & 0 & 0 & 0 & 0 \\
\hline 5 & 0 & 0 & 0 & 0 & 0 & 0 & 0 & 0 & 0 & 0 & 0 & 0 \\
\hline 6 & 0 & 0 & 0 & 0 & 0 & 0 & 0 & 0 & 0 & 0 & 0 & 0 \\
\hline 7 & 0 & 0 & 0 & 0 & 0 & 0 & 0 & 0 & 0 & 0 & 0 & 0 \\
\hline 8 & 0 & 0 & 0 & 0 & 0 & 0 & 0 & 0 & 0 & 0 & 0 & 0 \\
\hline 9 & 0 & 0 & 0 & 0 & 0 & 0 & 0 & 0 & 0 & 0 & 0 & 0 \\
\hline 10 & 0 & 0 & 0 & 0 & 0 & 0 & 0 & 0 & 0 & 0 & 0 & 0 \\
\hline 11 & 0 & 0 & 0 & 0 & 0 & 0 & 0 & 0 & 0 & 0 & 0 & 0 \\
\hline 12 & 0 & 0 & 0 & 0 & 0 & 0 & 0 & 0 & 0 & .62 & 2.2 & 0 \\
\hline 13 & 0 & 0 & 0 & 0 & 0 & 0 & 0 & 0 & 0 & .18 & 3.9 & 0 \\
\hline 14 & 0 & 0 & 0 & 0 & 0 & 0 & 0 & 0 & 0 & 0 & .53 & 0 \\
\hline 15 & 0 & 0 & 0 & 0 & 0 & 0 & 0 & 0 & 0 & 0 & 0 & 0 \\
\hline 16 & 0 & 0 & 0 & 0 & 0 & 0 & 0 & 0 & 0 & 0 & .04 & 0 \\
\hline 17 & 0 & 0 & 0 & 0 & 0 & 0 & 0 & 0 & 0 & 0 & 0 & 0 \\
\hline 18 & 0 & 0 & 0 & 0 & 0 & 0 & 0 & 0 & 0 & 0 & .66 & 0 \\
\hline 19 & 0 & 0 & 0 & 0 & 0 & 0 & 0 & 0 & 0 & .11 & 3.1 & 0 \\
\hline 20 & 0 & 0 & 0 & 0 & 0 & 0 & 0 & 0 & 0 & 0 & 2.2 & 0 \\
\hline 21 & 0 & 0 & 0 & 0 & 0 & 0 & 0 & 0 & 0 & 0 & 1.3 & 0 \\
\hline 22 & 0 & 0 & 0 & 0 & 0 & 0 & 0 & 0 & 0 & 0 & 1.3 & 0 \\
\hline 23 & 0 & 0 & 0 & 0 & 0 & 0 & 0 & 0 & 0 & 0 & .09 & 0 \\
\hline 24 & 0 & 0 & 0 & 0 & 0 & 0 & 0 & 0 & 0 & 0 & 0 & 0 \\
\hline 25 & 0 & 0 & 0 & 0 & 0 & 0 & 0 & 0 & 0 & 0 & 0 & 0 \\
\hline 26 & 0 & 0 & 0 & 0 & 0 & 0 & 0 & 0 & 0 & 0 & 0 & 0 \\
\hline 27 & 0 & 0 & 0 & 0 & 0 & 0 & 0 & 0 & 0 & 0 & 0 & 0 \\
\hline 28 & 0 & 0 & 0 & 0 & 0 & 0 & 0 & 0 & 11 & 0 & 0 & 0 \\
\hline 29 & 0 & 0 & 0 & 0 & ------ & 0 & 0 & 0 & .34 & 1.6 & 1.6 & 0 \\
\hline 30 & 0 & 0 & 0 & 0 & ------ & 0 & 0 & 0 & 0 & .34 & .04 & 0 \\
\hline 31 & 0 & ------ & 0 & 0 & ----- & 0 & ----- & $0^{*}$ & ----- & 0 & 0 & ----- \\
\hline Total & 0 & 0 & 0 & 0 & 0 & 0 & 0 & 0 & 11.34 & 2.85 & 16.96 & 0 \\
\hline Mean & 0 & 0 & 0 & 0 & 0 & 0 & 0 & 0 & .38 & .092 & .55 & 0 \\
\hline Max & 0 & 0 & 0 & 0 & 0 & 0 & 0 & 0 & 11 & 1.6 & 3.9 & 0 \\
\hline Min & 0 & 0 & 0 & 0 & 0 & 0 & 0 & 0 & 0 & 0 & 0 & 0 \\
\hline$A c-F t$ & 0 & 0 & 0 & 0 & 0 & 0 & 0 & 0 & 22 & 5.6 & 34 & 0 \\
\hline Wtr Year & 2000 & Total & & & Mean & .085 & Max & 11 & Min & 0 & Ac- $\mathrm{Ft}$ & 61 \\
\hline Cal Year & 1999 & Total & & 82 & Mean & .003 & Max & .40 & Min & 0 & $\mathrm{Ac}-\mathrm{Ft}$ & 1.6 \\
\hline
\end{tabular}




\section{E265 Water Canyon below Highway 4 near White Rock, NM}

Location. Lat 3548'17.7", long 106²'14'31.6" in Ramon Vigil Grant, Los Alamos County, $4.0 \mathrm{mi}$ SW of White Rock and 0.4 mi downstream from NM State Highway 4.

Drainage Area. $8.70 \mathrm{mi}^{2}$ (revised).

Period of Record. October 1993 through September 30, 2000.

Gage. Data logger with cellular telemetry and stabilized natural rock control. Elevation of gage is 6,314 ft above National Geodetic Vertical Datum of 1929, from GPS survey.

Remarks. Water discharge records fair.

Extremes for Period of Record. Maximum discharge, $274 \mathrm{ft}^{3} / \mathrm{s}$, gage height $5.13 \mathrm{ft}$ (from floodmark), June 28, 2000. No flow most of time.

Extremes for Current Year. Maximum discharge $274 \mathrm{ft}^{3} / \mathrm{s}$ at about $1400 \mathrm{hrs,} \mathrm{June} \mathrm{28,} \mathrm{gage}$ height $5.13 \mathrm{ft}$ (from floodmark). No flow most of time.

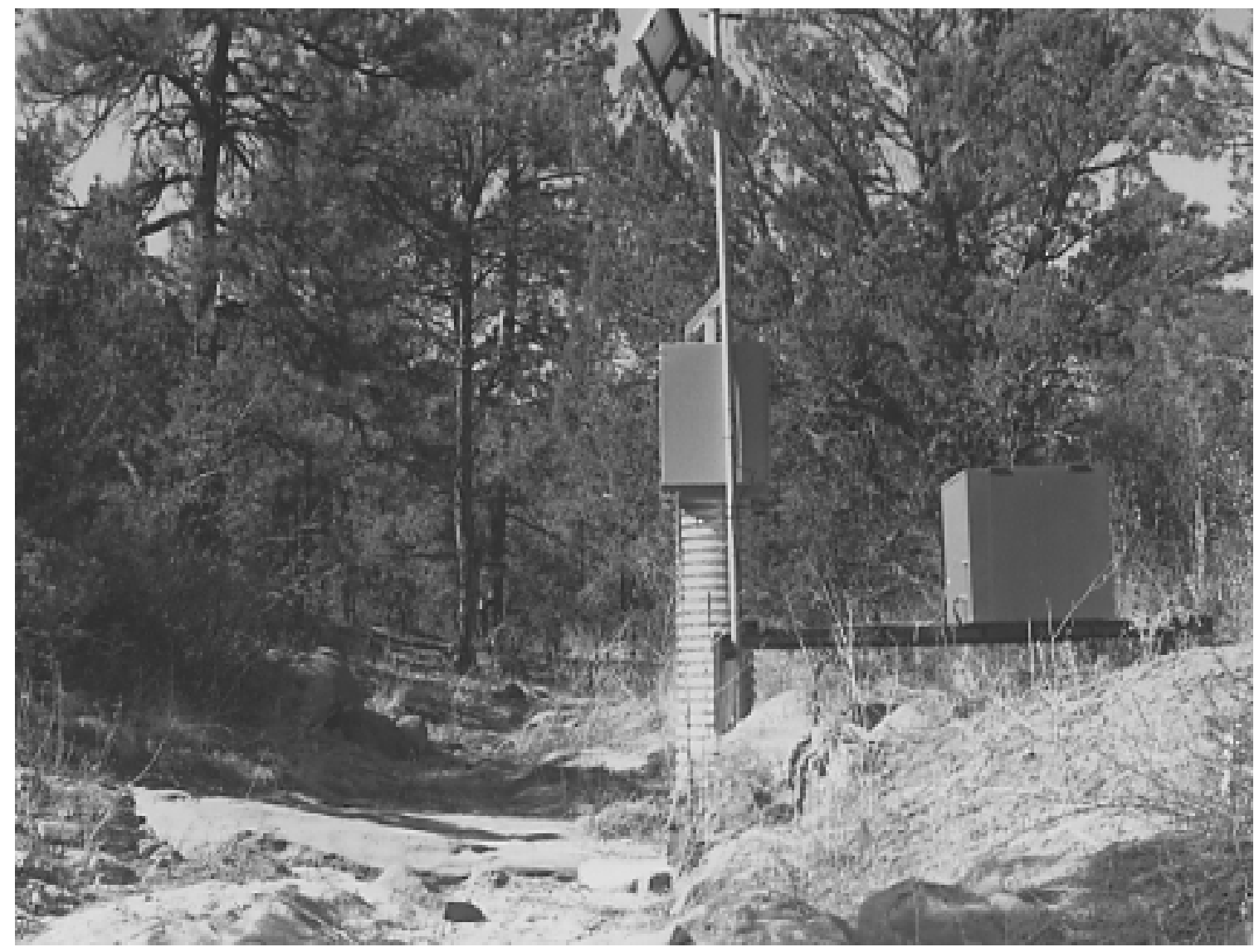




\section{E265 Water Canyon below Highway 4 near White Rock, NM}

Daily Mean Discharge in Cubic Feet per Second

Water Year October 1999 to September 2000

\begin{tabular}{|c|c|c|c|c|c|c|c|c|c|c|c|c|}
\hline DAY & OCT & NOV & DEC & JAN & FEB & MAR & APR & MAY & JUN & JUL & AUG & SEP \\
\hline 1 & 0 & 0 & 0 & 0 & 0 & 0 & 0 & 0 & 0 & 0 & 0 & 0 \\
\hline 2 & 0 & 0 & 0 & 0 & 0 & 0 & 0 & 0 & 0 & 0 & 0 & 0 \\
\hline 3 & 0 & 0 & 0 & 0 & 0 & 0 & 0 & 0 & 0 & 0 & 0 & 0 \\
\hline 4 & 0 & 0 & 0 & 0 & 0 & 0 & 0 & 0 & 0 & 0 & 0 & 0 \\
\hline 5 & 0 & 0 & 0 & 0 & 0 & 0 & 0 & 0 & 0 & 0 & 0 & 0 \\
\hline 6 & 0 & 0 & 0 & 0 & 0 & 0 & 0 & 0 & 0 & 0 & 0 & 0 \\
\hline 7 & 0 & 0 & 0 & 0 & 0 & 0 & 0 & 0 & 0 & 0 & 0 & 0 \\
\hline 8 & 0 & 0 & 0 & 0 & 0 & 0 & 0 & 0 & 0 & 0 & 0 & 0 \\
\hline 9 & 0 & 0 & 0 & 0 & 0 & 0 & 0 & 0 & 0 & 0 & 0 & 0 \\
\hline 10 & 0 & 0 & 0 & 0 & 0 & 0 & 0 & 0 & 0 & 0 & 0 & 0 \\
\hline 11 & 0 & 0 & 0 & 0 & 0 & 0 & 0 & 0 & 0 & 0 & .01 & 0 \\
\hline 12 & 0 & 0 & 0 & 0 & 0 & 0 & 0 & 0 & 0 & .41 & 1.4 & 0 \\
\hline 13 & 0 & 0 & 0 & 0 & 0 & 0 & 0 & 0 & 0 & .16 & 3.5 & 0 \\
\hline 14 & 0 & 0 & 0 & 0 & 0 & 0 & 0 & 0 & 0 & 0 & .43 & 0 \\
\hline 15 & 0 & 0 & 0 & 0 & 0 & 0 & 0 & 0 & 0 & 0 & 0 & 0 \\
\hline 16 & 0 & 0 & 0 & 0 & 0 & 0 & 0 & 0 & 0 & 0 & .02 & 0 \\
\hline 17 & 0 & 0 & 0 & 0 & 0 & 0 & 0 & 0 & .14 & 0 & 0 & 0 \\
\hline 18 & 0 & 0 & 0 & 0 & 0 & 0 & 0 & 0 & 0 & 0 & .12 & 0 \\
\hline 19 & 0 & 0 & 0 & 0 & 0 & 0 & 0 & 0 & 0 & .08 & 3.1 & 0 \\
\hline 20 & 0 & 0 & 0 & 0 & 0 & 0 & 0 & 0 & 0 & 0 & 3.3 & 0 \\
\hline 21 & 0 & 0 & 0 & 0 & 0 & 0 & 0 & 0 & 0 & 0 & .66 & 0 \\
\hline 22 & 0 & 0 & 0 & 0 & 0 & 0 & 0 & 0 & 0 & 0 & .27 & 0 \\
\hline 23 & 0 & 0 & 0 & 0 & 0 & 0 & 0 & 0 & 0 & 0 & .15 & 0 \\
\hline 24 & 0 & 0 & 0 & 0 & 0 & 0 & 0 & 0 & 0 & 0 & .01 & 0 \\
\hline 25 & 0 & 0 & 0 & 0 & 0 & 0 & 0 & 0 & 0 & 0 & 0 & 0 \\
\hline 26 & 0 & 0 & 0 & 0 & 0 & 0 & 0 & 0 & 0 & 0 & 0 & 0 \\
\hline 27 & 0 & 0 & 0 & 0 & 0 & 0 & 0 & 0 & 0 & 0 & 0 & 0 \\
\hline 28 & 0 & 0 & 0 & 0 & 0 & 0 & 0 & 0 & 11 & 0 & 0 & 0 \\
\hline 29 & 0 & 0 & 0 & 0 & ------ & 0 & 0 & 0 & .38 & .81 & 1.0 & 0 \\
\hline 30 & 0 & 0 & 0 & 0 & ------ & 0 & 0 & 0 & 0 & .40 & .10 & 0 \\
\hline 31 & 0 & ------ & 0 & 0 & ------ & 0 & ------ & 0 & ------ & .07 & 0 & ------ \\
\hline Total & 0 & 0 & 0 & 0 & 0 & 0 & 0 & 0 & 11.38 & 1.93 & 14.07 & 0 \\
\hline Mean & 0 & 0 & 0 & 0 & 0 & 0 & 0 & 0 & .38 & .062 & .45 & 0 \\
\hline Max & 0 & 0 & 0 & 0 & 0 & 0 & 0 & 0 & 11 & .81 & 3.5 & 0 \\
\hline Min & 0 & 0 & 0 & 0 & 0 & 0 & 0 & 0 & 0 & 0 & 0 & 0 \\
\hline Ac-Ft & 0 & 0 & 0 & 0 & 0 & 0 & 0 & 0 & 22 & 3.8 & 28 & 0 \\
\hline Wtr Year & 2000 & Total & & & Mean & .075 & Max & 11 & Min & 0 & $\mathrm{Ac}-\mathrm{Ft}$ & 53 \\
\hline Cal Year & 1999 & Total & & 22 & Mean & .001 & $\operatorname{Max}$ & .14 & Min & 0 & $\mathrm{Ac}-\mathrm{Ft}$ & .4 \\
\hline
\end{tabular}




\section{E267 Potrillo Canyon near White Rock, NM}

Location. Lat $35^{\circ} 48^{\prime} 48^{\prime \prime}$, long $106^{\circ} 14^{\prime} 00^{\prime \prime}$, in Ramon Vigil Grant, Los Alamos County, $2.0 \mathrm{mi}$ SW of White Rock and 0.25 mi upstream from NM State Highway 4.

Drainage Area. $2.25 \mathrm{mi}^{2}$.

Period of Record. October 1993 to September 30, 2000.

Gage. Data logger with cellular telemetry and concrete control. Elevation of gage is $6,458 \mathrm{ft}$ above National Geodetic Vertical Datum of 1929, from GPS survey.

Remarks. Water discharge records good.

Revisions. Station number; LA-13551-PR (1998).

Extremes for Period of Record. Maximum discharge $63 \mathrm{ft}^{3} / \mathrm{s}$, August 29, 1995, gage height $2.70 \mathrm{ft}$ (from slope-area determination). No flow most of time.

Extremes for Current Water Year. Maximum discharge $7 \mathrm{ft}^{3} / \mathrm{s}$ at $2055 \mathrm{hrs,} \mathrm{August} \mathrm{9,} \mathrm{gage}$ height $1.35 \mathrm{ft}$. No flow most of time.

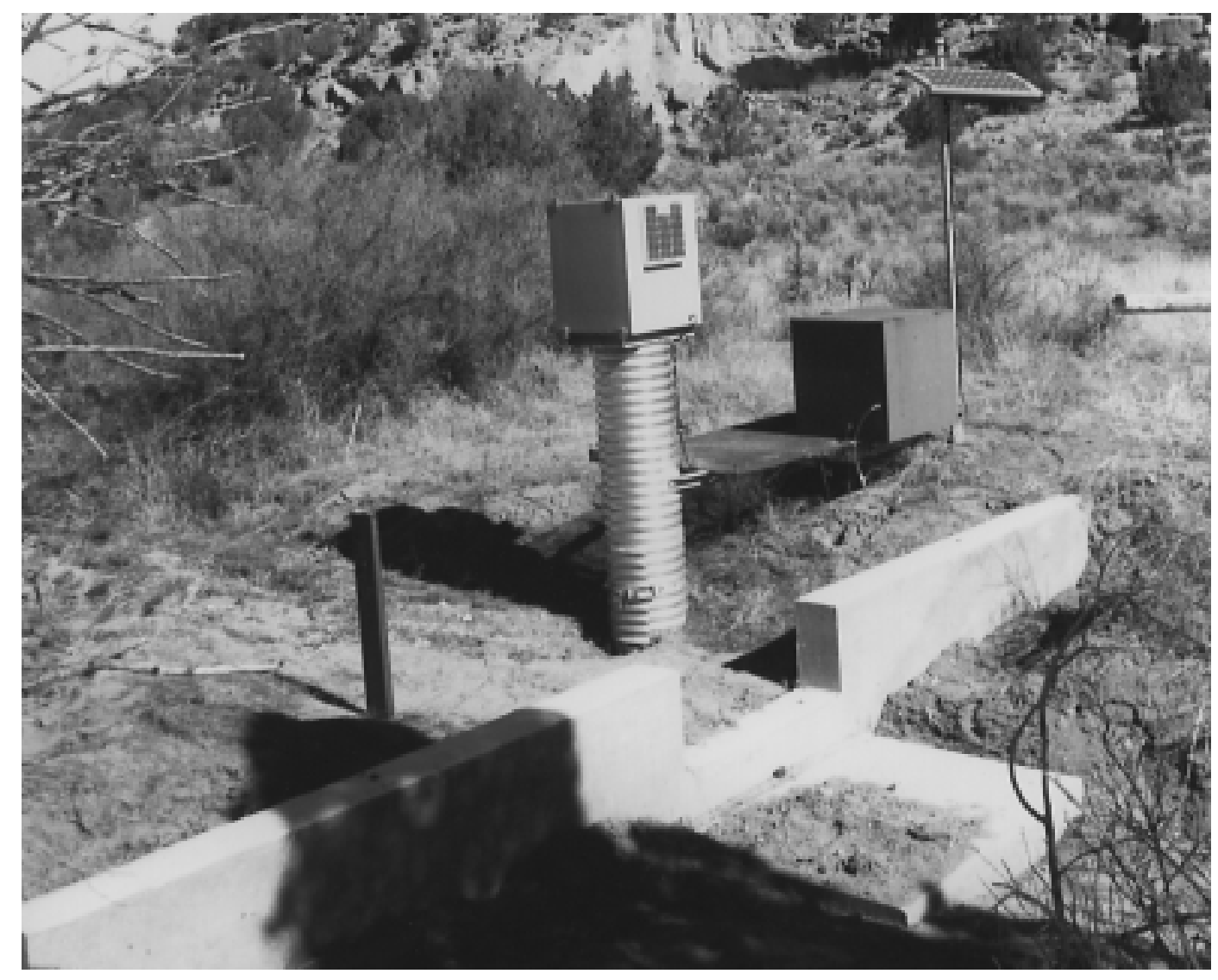




\section{E267 Potrillo Canyon near White Rock, NM}

Daily Mean Discharge in Cubic Feet per Second

Water Year October 1999 to September 2000

\begin{tabular}{|c|c|c|c|c|c|c|c|c|c|c|c|c|}
\hline DAY & ОСТ & NOV & DEC & JAN & FEB & MAR & APR & MAY & JUN & JUL & AUG & SEP \\
\hline 1 & 0 & 0 & 0 & 0 & 0 & 0 & 0 & 0 & 0 & 0 & 0 & 0 \\
\hline 2 & 0 & 0 & 0 & 0 & 0 & 0 & 0 & 0 & 0 & 0 & 0 & 0 \\
\hline 3 & 0 & 0 & 0 & 0 & 0 & 0 & 0 & 0 & 0 & 0 & .07 & 0 \\
\hline 4 & 0 & 0 & 0 & 0 & 0 & 0 & 0 & 0 & 0 & 0 & 0 & 0 \\
\hline 5 & 0 & 0 & 0 & 0 & 0 & 0 & 0 & 0 & 0 & 0 & 0 & 0 \\
\hline 6 & 0 & 0 & 0 & 0 & 0 & 0 & 0 & 0 & 0 & 0 & 0 & 0 \\
\hline 7 & 0 & 0 & 0 & 0 & 0 & 0 & 0 & 0 & 0 & 0 & 0 & 0 \\
\hline 8 & 0 & 0 & 0 & 0 & 0 & 0 & 0 & 0 & 0 & 0 & 0 & 0 \\
\hline 9 & 0 & 0 & 0 & 0 & 0 & 0 & 0 & 0 & 0 & 0 & .24 & 0 \\
\hline 10 & 0 & 0 & 0 & 0 & 0 & 0 & 0 & 0 & 0 & 0 & 0 & 0 \\
\hline 11 & 0 & 0 & 0 & 0 & 0 & 0 & 0 & 0 & 0 & 0 & 0 & 0 \\
\hline 12 & 0 & 0 & 0 & 0 & 0 & 0 & 0 & 0 & 0 & 0 & 0 & 0 \\
\hline 13 & 0 & 0 & 0 & 0 & 0 & 0 & 0 & 0 & 0 & 0 & 0 & 0 \\
\hline 14 & 0 & 0 & 0 & 0 & 0 & 0 & 0 & 0 & 0 & 0 & 0 & 0 \\
\hline 15 & 0 & 0 & 0 & 0 & 0 & 0 & 0 & 0 & 0 & 0 & 0 & 0 \\
\hline 16 & 0 & 0 & 0 & 0 & 0 & 0 & 0 & 0 & 0 & 0 & 0 & 0 \\
\hline 17 & 0 & 0 & 0 & 0 & 0 & 0 & 0 & 0 & 0 & 0 & 0 & 0 \\
\hline 18 & 0 & 0 & 0 & 0 & 0 & 0 & 0 & 0 & 0 & 0 & .01 & 0 \\
\hline 19 & 0 & 0 & 0 & 0 & 0 & 0 & 0 & 0 & 0 & 0 & .02 & 0 \\
\hline 20 & 0 & 0 & 0 & 0 & 0 & 0 & 0 & 0 & 0 & 0 & 0 & 0 \\
\hline 21 & 0 & 0 & 0 & 0 & 0 & 0 & 0 & 0 & 0 & 0 & 0 & 0 \\
\hline 22 & 0 & 0 & 0 & 0 & 0 & 0 & 0 & 0 & 0 & 0 & 0 & 0 \\
\hline 23 & 0 & 0 & 0 & 0 & 0 & 0 & 0 & 0 & 0 & 0 & 0 & 0 \\
\hline 24 & 0 & 0 & 0 & 0 & 0 & 0 & 0 & 0 & 0 & 0 & 0 & 0 \\
\hline 25 & 0 & 0 & 0 & 0 & 0 & 0 & 0 & 0 & 0 & 0 & 0 & 0 \\
\hline 26 & 0 & 0 & 0 & 0 & 0 & 0 & 0 & 0 & 0 & 0 & 0 & 0 \\
\hline 27 & 0 & 0 & 0 & 0 & 0 & 0 & 0 & 0 & 0 & 0 & 0 & 0 \\
\hline 28 & 0 & 0 & 0 & 0 & 0 & 0 & 0 & 0 & 0 & 0 & 0 & 0 \\
\hline 29 & 0 & 0 & 0 & 0 & ----- & 0 & 0 & 0 & 0 & .03 & 0 & 0 \\
\hline 30 & 0 & 0 & 0 & 0 & ----- & 0 & 0 & 0 & 0 & 0 & 0 & 0 \\
\hline 31 & 0 & ----- & 0 & 0 & ----- & 0 & ----- & 0 & ----- & 0 & 0 & $---\cdot$ \\
\hline Total & 0 & 0 & 0 & 0 & 0 & 0 & 0 & 0 & 0 & 0.03 & 0.34 & 0 \\
\hline Mean & 0 & 0 & 0 & 0 & 0 & 0 & 0 & 0 & 0 & .001 & .011 & 0 \\
\hline Max & 0 & 0 & 0 & 0 & 0 & 0 & 0 & 0 & 0 & .03 & .24 & 0 \\
\hline Min & 0 & 0 & 0 & 0 & 0 & 0 & 0 & 0 & 0 & 0 & 0 & 0 \\
\hline $\mathrm{Ac}-\mathrm{Ft}$ & 0 & 0 & 0 & 0 & 0 & 0 & 0 & 0 & 0 & .05 & .7 & 0 \\
\hline Wtr Year & 2000 & Total & & & Mean & .001 & Max & .24 & Min & 0 & $\mathrm{Ac}-\mathrm{Ft}$ & .7 \\
\hline Cal Year & 1999 & Total & c & & Mean & 0 & Max & 0 & Min & 0 & $\mathrm{Ac}-\mathrm{Ft}$ & 0 \\
\hline
\end{tabular}




\section{E275 Ancho Canyon near Bandelier National Monument, NM}

Location. Lat 3546'54.2", long 106¹4'41.9", in Ramon Vigil Grant, Los Alamos County, $5.5 \mathrm{mi} \mathrm{SW}$ of White Rock at $0.3 \mathrm{mi}$ downstream from NM State Highway 4.

Drainage Area. $4.55 \mathrm{mi}^{2}$.

Period of Record. December 1993 to September 30, 2000.

Gage. Data logger with cellular telemetry and concrete stabilized natural control. Elevation of gage is 6,198 ft above National Geodetic Vertical Datum of 1929, from GPS survey.

Remarks. Water discharge records fair.

Extremes for Period of Record. Maximum discharge $520 \mathrm{ft}^{3} / \mathrm{s}$, gage height $2.71 \mathrm{ft}$, June 29, 1995. No flow most of time.

Extremes for Current Year. Maximum discharge $349 \mathrm{ft}^{3} / \mathrm{s}$, gage height $2.35 \mathrm{ft}$ at $1630 \mathrm{hrs,}$ August 6. No flow most of time.

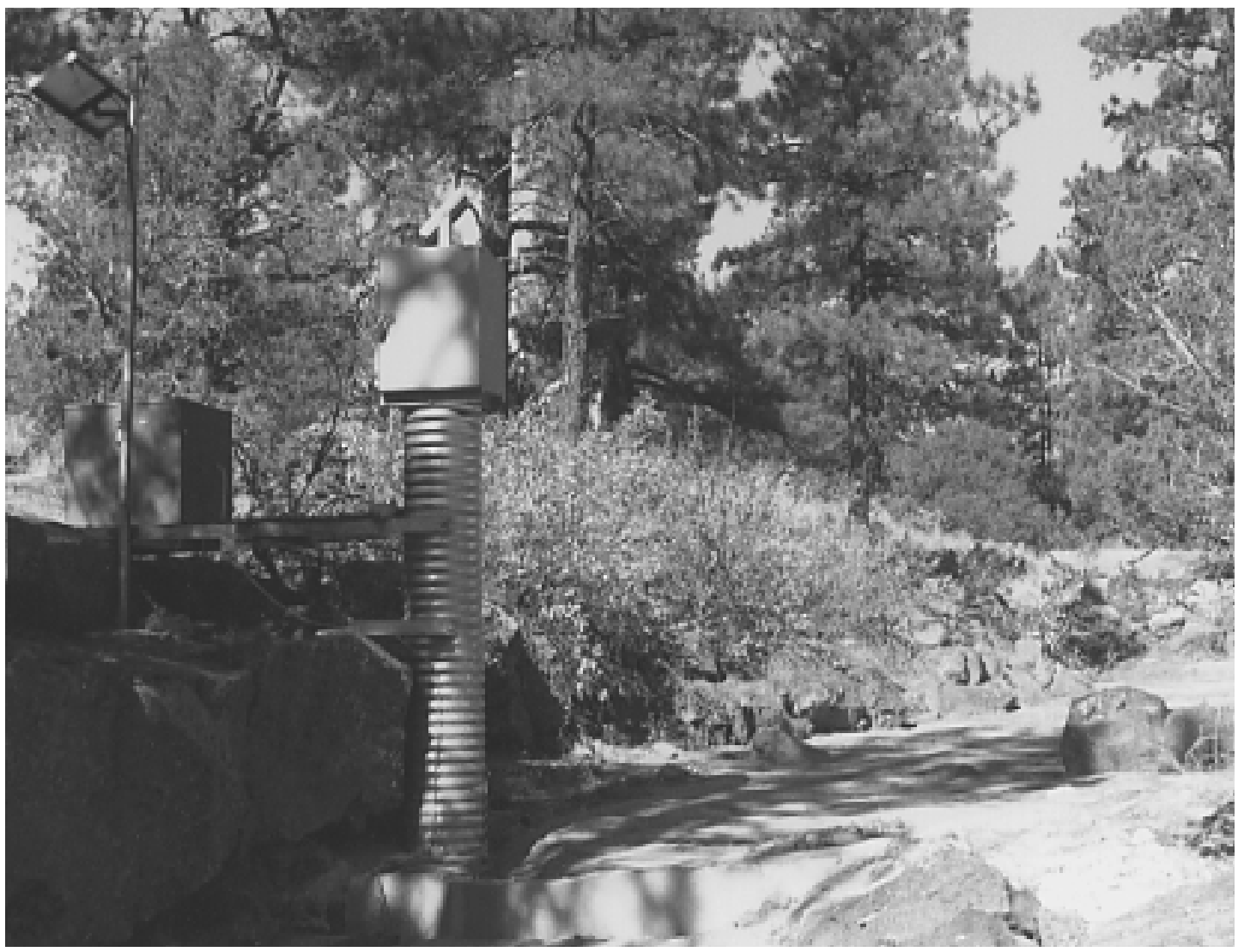


E275 Ancho Canyon near Bandelier National Monument, NM

Daily Mean Discharge in Cubic Feet per Second

Water Year October 1999 to September 2000

\begin{tabular}{|c|c|c|c|c|c|c|c|c|c|c|c|c|}
\hline DAY & OCT & NOV & DEC & JAN & FEB & MAR & APR & MAY & JUN & JUL & AUG & SEP \\
\hline 1 & 0 & 0 & 0 & 0 & 0 & 0 & 0 & 0 & 0 & 0 & 0 & 0 \\
\hline 2 & 0 & 0 & 0 & 0 & 0 & 0 & 0 & 0 & 0 & 0 & 0 & 0 \\
\hline 3 & 0 & 0 & 0 & 0 & 0 & 0 & 0 & 0 & 0 & 0 & 0 & 0 \\
\hline 4 & 0 & 0 & 0 & 0 & 0 & 0 & 0 & 0 & 0 & 0 & 0 & 0 \\
\hline 5 & 0 & 0 & 0 & 0 & 0 & 0 & 0 & 0 & 0 & 0 & 0 & 0 \\
\hline 6 & 0 & 0 & 0 & 0 & 0 & 0 & 0 & 0 & 0 & 0 & 3.0 & 0 \\
\hline 7 & 0 & 0 & 0 & 0 & 0 & 0 & 0 & 0 & 0 & 0 & 0 & 0 \\
\hline 8 & 0 & 0 & 0 & 0 & 0 & 0 & 0 & 0 & 0 & 0 & 0 & 0 \\
\hline 9 & 0 & 0 & 0 & 0 & 0 & 0 & 0 & 0 & 0 & 0 & .23 & 0 \\
\hline 10 & 0 & 0 & 0 & 0 & 0 & 0 & 0 & 0 & 0 & 0 & 0 & 0 \\
\hline 11 & 0 & 0 & 0 & 0 & 0 & 0 & 0 & 0 & 0 & 0 & 0 & 0 \\
\hline 12 & 0 & 0 & 0 & 0 & 0 & 0 & 0 & 0 & 0 & 0 & 0 & 0 \\
\hline 13 & 0 & 0 & 0 & 0 & 0 & 0 & 0 & 0 & 0 & 0 & 0 & 0 \\
\hline 14 & 0 & 0 & 0 & 0 & 0 & 0 & 0 & 0 & 0 & 0 & 0 & 0 \\
\hline 15 & 0 & 0 & 0 & 0 & 0 & 0 & 0 & 0 & 0 & 0 & 0 & 0 \\
\hline 16 & 0 & 0 & 0 & 0 & 0 & 0 & 0 & 0 & 0 & 0 & 0 & 0 \\
\hline 17 & 0 & 0 & 0 & 0 & 0 & 0 & 0 & 0 & 0 & 0 & 0 & 0 \\
\hline 18 & 0 & 0 & 0 & 0 & 0 & 0 & 0 & 0 & 0 & 0 & .19 & 0 \\
\hline 19 & 0 & 0 & 0 & 0 & 0 & 0 & 0 & 0 & 0 & 0 & .65 & 0 \\
\hline 20 & 0 & 0 & 0 & 0 & 0 & 0 & 0 & 0 & 0 & 0 & .16 & 0 \\
\hline 21 & 0 & 0 & 0 & 0 & 0 & 0 & 0 & 0 & 0 & 0 & 0 & 0 \\
\hline 22 & 0 & 0 & 0 & 0 & 0 & 0 & 0 & 0 & 0 & 0 & 0 & 0 \\
\hline 23 & 0 & 0 & 0 & 0 & 0 & 0 & 0 & 0 & 0 & 0 & 0 & 0 \\
\hline 24 & 0 & 0 & 0 & 0 & 0 & 0 & 0 & 0 & 0 & 0 & 0 & 0 \\
\hline 25 & 0 & 0 & 0 & 0 & 0 & 0 & 0 & 0 & 0 & 0 & 0 & 0 \\
\hline 26 & 0 & 0 & 0 & 0 & 0 & 0 & 0 & 0 & 0 & 0 & 0 & 0 \\
\hline 27 & 0 & 0 & 0 & 0 & 0 & 0 & 0 & 0 & 0 & 0 & 0 & 0 \\
\hline 28 & 0 & 0 & 0 & 0 & 0 & 0 & 0 & 0 & 0 & 0 & 0 & 0 \\
\hline 29 & 0 & 0 & 0 & 0 & ------ & 0 & 0 & 0 & 0 & .10 & 0 & 0 \\
\hline 30 & 0 & 0 & 0 & 0 & ------ & 0 & 0 & 0 & 0 & 0 & 0 & 0 \\
\hline 31 & 0 & ------ & 0 & 0 & ------ & 0 & ------ & 0 & -.---- & 0 & 0 & ------ \\
\hline Total & 0 & 0 & 0 & 0 & 0 & 0 & 0 & 0 & 0 & .10 & 4.23 & 0 \\
\hline Mean & 0 & 0 & 0 & 0 & 0 & 0 & 0 & 0 & 0 & .003 & .14 & 0 \\
\hline Max & 0 & 0 & 0 & 0 & 0 & 0 & 0 & 0 & 0 & .10 & 3.0 & 0 \\
\hline Min & 0 & 0 & 0 & 0 & 0 & 0 & 0 & 0 & 0 & 0 & 0 & 0 \\
\hline Ac-Ft & 0 & 0 & 0 & 0 & 0 & 0 & 0 & 0 & 0 & .2 & 8.4 & 0 \\
\hline Wtr Year & 2000 & Total & & & lean & .012 & $\operatorname{Max}$ & 3.0 & Min & 0 & $\mathrm{Ac}-\mathrm{Ft}$ & 8.6 \\
\hline Cal Year & 1999 & Total & & & lean & .014 & Max & 2.0 & Min & 0 & Ac-Ft & 10 \\
\hline
\end{tabular}




\section{E350 Rio de los Frijoles at Bandelier}

Location. Lat 3546'37.0", long 106²' $6^{\prime} 9.6^{\prime \prime}$, Ramon Vigil Grant, Sandoval County, in Bandelier National Monument, on right bank $800 \mathrm{ft}$ downstream from Monument Headquarters, $6.5 \mathrm{mi} \mathrm{S}$ of Los Alamos, $18.5 \mathrm{mi} \mathrm{NW}$ of Santa Fe, and at mile 2.0.

Drainage Area. $18.16 \mathrm{mi}^{2}$.

Period of Record. July 1963 to September 1969, July 1977 to September 1982, May 1993 to September 1996, and October 1998 to September 30, 2000.

Gage. Data logger and concrete control. Elevation of gage is 6,046 ft above National Geodetic Vertical Datum of 1929, from GPS Survey.

Remarks. Water discharge records fair except those for winter period, which are poor. One small diversion from left bank about 1.0 mi upstream for irrigation of small orchard. The La Mesa fire, which occurred during mid June 1977, burned about $40 \%$ of the forest cover of this watershed.

Extremes for Period of Record. Maximum discharge 3,030 $\mathrm{ft}^{3} / \mathrm{s}$, July 21, 1978, gage height $6.34 \mathrm{ft}$, site and datum then in use. Minimum daily discharge $0.15 \mathrm{ft}^{3} / \mathrm{s}$ July 9, 1989.

Extremes for Current Water Year. Maximum discharge $40 \mathrm{ft}^{3} / \mathrm{s}$, at $1325 \mathrm{hrs,} \mathrm{June} \mathrm{28,} \mathrm{gage}$ height $2.66 \mathrm{ft}$. Minimum daily $0.22 \mathrm{ft}^{3} / \mathrm{s}$, July 7 .

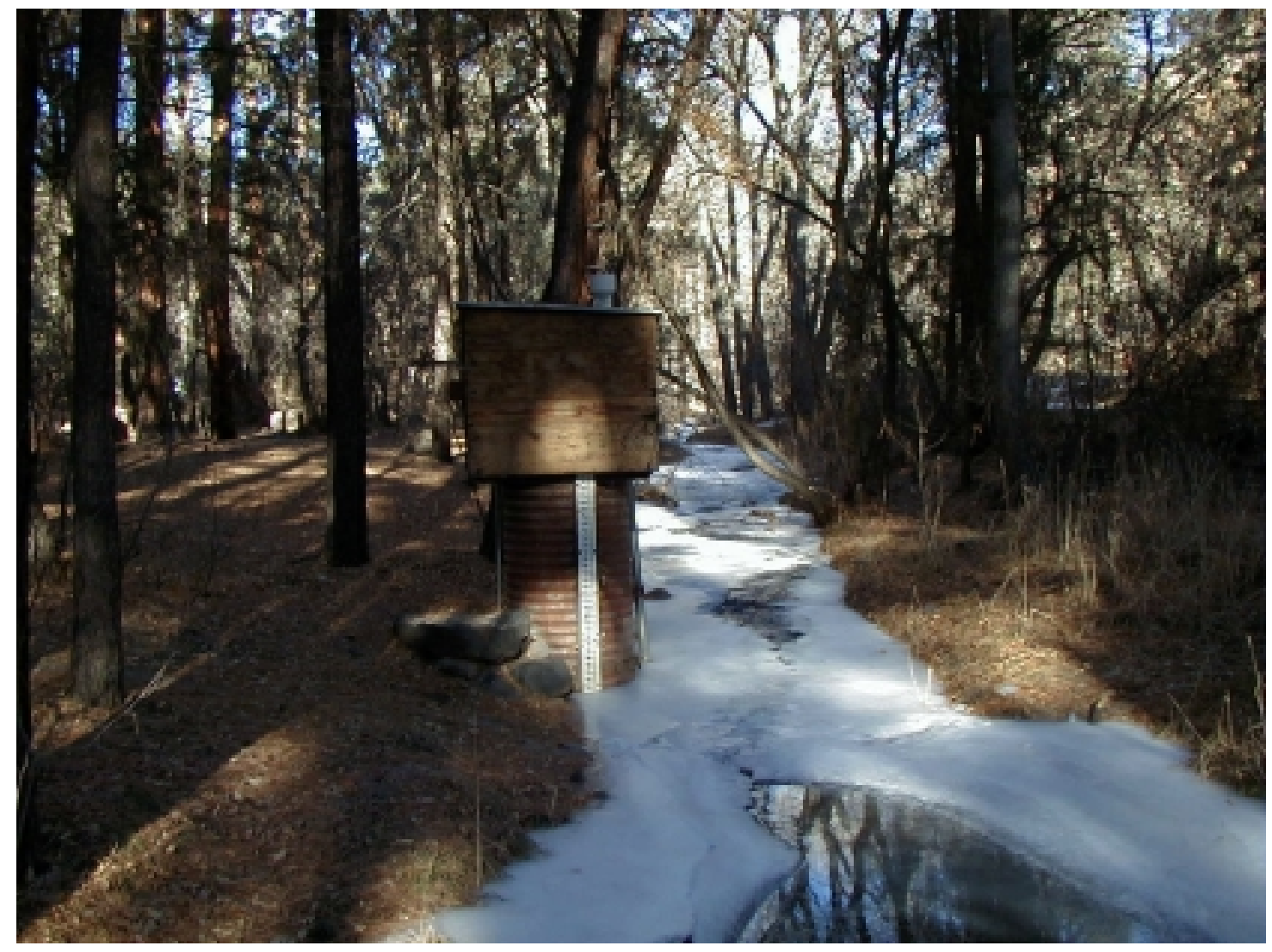




\section{E350 Rio de los Frijoles at Bandelier}

Daily Mean Discharge in Cubic Feet per Second

Water Year October 1999 to September 2000

\begin{tabular}{|c|c|c|c|c|c|c|c|c|c|c|c|c|}
\hline DAY & OCT & NOV & DEC & JAN & FEB & MAR & APR & MAY & JUN & JUL & AUG & SEP \\
\hline 1 & .74 & .81 & .70 & .73 & 1.3 & .98 & 1.2 & .96 & .33 & .42 & .35 & .40 \\
\hline 2 & .66 & .81 & .70 & .75 & 3.3 & .98 & 1.2 & .90 & .36 & .35 & .33 & .38 \\
\hline 3 & .66 & .81 & .70 & .74 & 2.4 & .98 & 1.2 & .85 & .50 & .40 & .32 & .36 \\
\hline 4 & .66 & .81 & .70 & .70 & 1.1 & .98 & 1.1 & .81 & .46 & .30 & .34 & .33 \\
\hline 5 & .66 & .81 & .70 & .65 & 1.2 & .98 & 1.1 & .76 & .42 & .25 & .39 & .30 \\
\hline 6 & .66 & .81 & .70 & .63 & 1.2 & .98 & 1.1 & .74 & .46 & .23 & .36 & .29 \\
\hline 7 & .66 & .81 & .70 & .60 & 1.1 & 1.0 & 1.1 & .72 & .42 & .22 & .35 & .29 \\
\hline 8 & .66 & .81 & .70 & .60 & 1.1 & 1.0 & 1.1 & .70 & .37 & .23 & .34 & .31 \\
\hline 9 & .66 & .81 & .70 & .65 & 1.0 & .98 & 1.1 & .71 & .49 & .23 & .52 & .45 \\
\hline 10 & .66 & .81 & .65 & .81 & 1.0 & .98 & 1.1 & .66 & .44 & .41 & .49 & .35 \\
\hline 11 & .66 & .81 & .65 & 1.2 & 1.0 & .99 & 1.1 & .62 & .36 & .39 & .33 & .31 \\
\hline 12 & .66 & .81 & .65 & 1.1 & 1.0 & .98 & 1.2 & .61 & .33 & .46 & .38 & .30 \\
\hline 13 & .66 & .81 & .60 & 1.0 & 1.0 & .98 & 1.1 & .63 & .31 & .53 & .70 & .29 \\
\hline 14 & .66 & .81 & .60 & 1.0 & 1.0 & .98 & 1.1 & .64 & .30 & .42 & .75 & .28 \\
\hline 15 & .66 & .81 & .60 & 1.1 & 1.0 & .96 & 1.1 & .61 & .29 & .43 & .45 & .27 \\
\hline 16 & .71 & .81 & .60 & .99 & .98 & .97 & 1.1 & .58 & .27 & .37 & .49 & .28 \\
\hline 17 & .79 & .81 & .60 & 1.0 & .99 & .98 & 1.1 & .59 & .28 & .46 & .43 & .26 \\
\hline 18 & .80 & .81 & .60 & 1.0 & .98 & .98 & 1.0 & .58 & .28 & .64 & 1.0 & .29 \\
\hline 19 & .80 & .81 & .60 & 1.0 & .98 & .95 & 1.0 & .62 & .36 & .65 & 1.5 & .31 \\
\hline 20 & .80 & .80 & .60 & .98 & .98 & .98 & 1.0 & .64 & .31 & .51 & 1.1 & .31 \\
\hline 21 & .81 & .80 & .60 & 1.0 & .98 & 1.0 & .99 & .58 & .27 & .48 & .75 & .37 \\
\hline 22 & .81 & .79 & .65 & .98 & .98 & 1.3 & .98 & .51 & .28 & .46 & .68 & .45 \\
\hline 23 & .81 & .79 & .68 & 1.1 & .98 & 1.2 & .97 & .47 & .37 & .40 & .59 & .36 \\
\hline 24 & .82 & .78 & .68 & 1.2 & .98 & 1.2 & .94 & .45 & .31 & .39 & .54 & .35 \\
\hline 25 & .82 & .78 & .70 & 1.0 & .98 & 1.1 & .92 & .45 & .28 & .40 & .50 & .40 \\
\hline 26 & .83 & .78 & .70 & 1.1 & 1.1 & 1.1 & .89 & .45 & .27 & .53 & .48 & .42 \\
\hline 27 & .83 & .75 & .70 & 1.1 & 1.2 & 1.1 & .85 & .42 & .40 & .49 & .46 & .38 \\
\hline 28 & .83 & .70 & .70 & 1.0 & 1.1 & 1.1 & .87 & .40 & 2.5 & .40 & .43 & .36 \\
\hline 29 & .84 & .70 & .70 & 1.0 & ----- & 1.1 & .86 & .37 & .67 & .48 & .43 & .36 \\
\hline 30 & .76 & .70 & .70 & .95 & ------ & 1.1 & .87 & .35 & .48 & .53 & .43 & .37 \\
\hline 31 & .76 & ----- & .72 & 1.0 & ------ & 1.1 & ----- & .33 & ----- & .40 & .42 & ----- \\
\hline Total & 22.80 & 23.76 & 20.58 & 28.66 & 33.91 & 31.99 & 31.24 & 18.71 & 13.17 & 12.86 & 16.63 & 10.18 \\
\hline Mean & .74 & .79 & .66 & .92 & 1.17 & 1.03 & 1.04 & .60 & .44 & .41 & .54 & .34 \\
\hline Max & .84 & .81 & .72 & 1.2 & 3.3 & 1.3 & 1.2 & .96 & 2.5 & .65 & 1.5 & .45 \\
\hline Min & .66 & .70 & .60 & .60 & .98 & .95 & .85 & .33 & .27 & .22 & .32 & .26 \\
\hline Ac-Ft & 45 & 47 & 41 & 57 & 68 & 63 & 62 & 37 & 26 & 26 & 34 & 20 \\
\hline Wtr Year & 2000 & Tota & & & Mean & .72 & Max & 3.3 & Min & .22 & $\mathrm{Ac}-\mathrm{Ft}$ & 526 \\
\hline Cal Year & 1999 & Tota & 30 & & Mean & .82 & Max & 4.0 & Min & .26 & Ac-Ft & 595 \\
\hline
\end{tabular}



Spring Stations 



\section{S001 SWSC Line Spring at TA-16}

Location. Lat $35^{\circ} 51^{\prime} 1 "$, long $106^{\circ} 20^{\prime} 23^{\prime \prime}, 30 \mathrm{ft}$ upstream from the sanitary wastewater system consolidation (SWSC) line crossing of Cañon del Valle in Laboratory TA-16.

Gage. Data logger with $90^{\circ}$ weir. Elevation of gage is $7,437.0 \mathrm{ft}$ above National Geodetic Vertical Datum of 1929, from GPS survey.

Period of Record. October 1, 1996, to September 30, 2000.

Remarks. Water discharge records good. This spring is in the Cañon del Valle drainage.

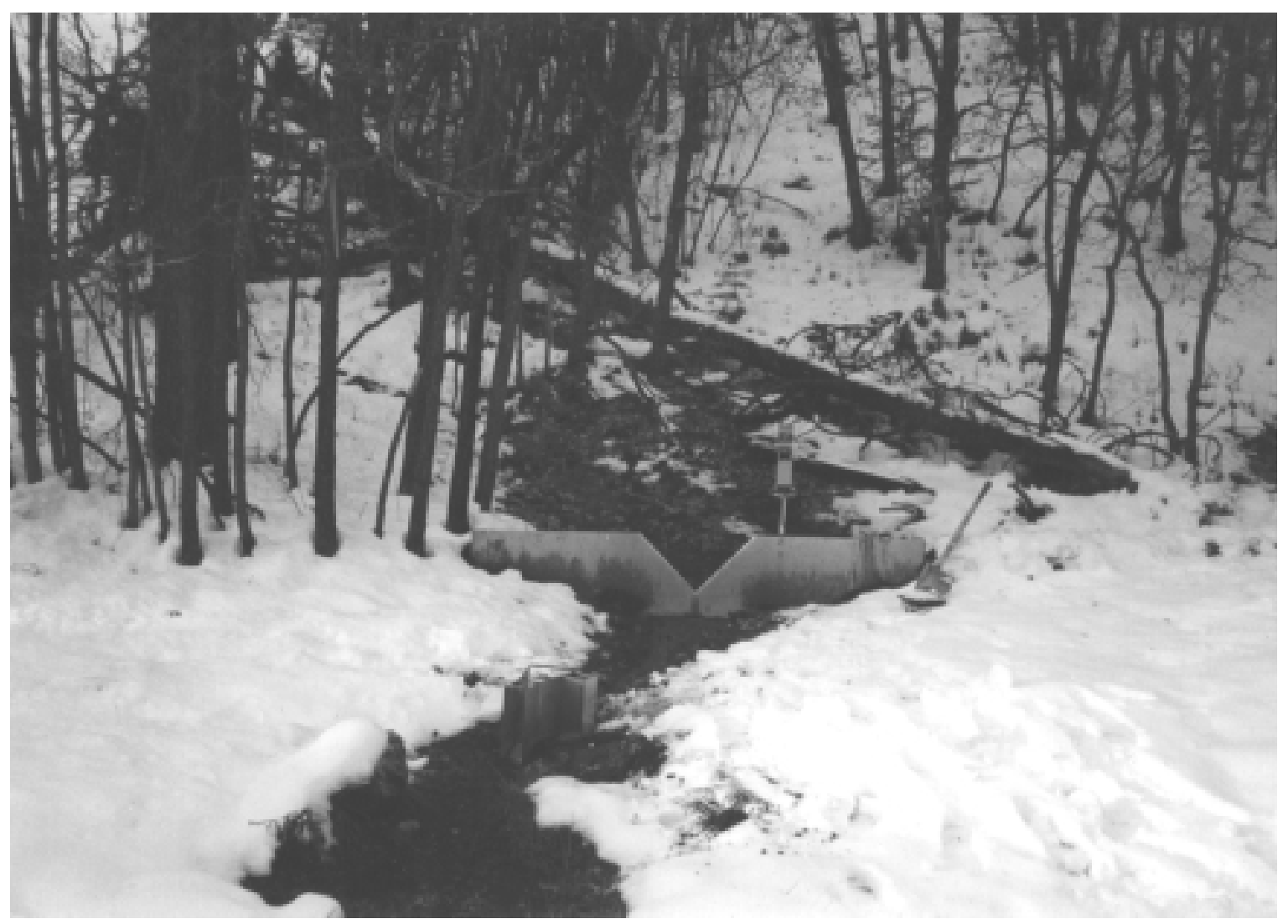




\section{S001 SWSC Line Spring at TA-16}

Daily Mean Discharge in Cubic Feet per Second

Water Year October 1999 to September 2000

\begin{tabular}{|c|c|c|c|c|c|c|c|c|c|c|c|c|}
\hline DAY & OCT & NOV & DEC & JAN & FEB & MAR & APR & MAY & JUN & JUL & AUG & SEP \\
\hline 1 & .009 & .006 & .004 & .006 & .004 & .007 & .003 & .003 & .002 & .002 & .003 & .004 \\
\hline 2 & .008 & .006 & .005 & .006 & .004 & .007 & .003 & .003 & .002 & .002 & .003 & .004 \\
\hline 3 & .005 & .006 & .008 & .006 & .004 & .005 & .003 & .003 & .002 & .001 & .003 & .004 \\
\hline 4 & .005 & .006 & .007 & .007 & .004 & .005 & .003 & .003 & .002 & .002 & .003 & .004 \\
\hline 5 & .005 & .005 & .006 & .009 & .004 & .005 & .003 & .002 & .002 & .002 & .004 & .004 \\
\hline 6 & .006 & .005 & .006 & .008 & .004 & .005 & .003 & .002 & .002 & .003 & .003 & .004 \\
\hline 7 & .009 & .005 & .006 & .006 & .004 & .005 & .003 & .002 & .002 & .003 & .003 & .004 \\
\hline 8 & .007 & .005 & .005 & .005 & .004 & .007 & .003 & .002 & .002 & .003 & .003 & .004 \\
\hline 9 & .006 & .006 & .004 & .009 & .004 & .008 & .003 & .002 & .003 & .003 & .003 & .004 \\
\hline 10 & .007 & .005 & .004 & .010 & .004 & .007 & .003 & .002 & .003 & .003 & .003 & .004 \\
\hline 11 & .005 & .005 & .005 & .012 & .004 & .005 & .003 & .002 & .003 & .003 & .003 & .004 \\
\hline 12 & .007 & .005 & .004 & .015 & .004 & .005 & .003 & .002 & .002 & .003 & .003 & .004 \\
\hline 13 & .006 & .005 & .004 & .013 & .004 & .004 & .003 & .003 & .003 & .003 & .003 & .004 \\
\hline 14 & .006 & .005 & .005 & .008 & .004 & .004 & .003 & .003 & .003 & .003 & .003 & .004 \\
\hline 15 & .006 & .005 & .005 & .008 & .004 & .005 & .003 & .002 & .002 & .003 & .004 & .004 \\
\hline 16 & .008 & .005 & .005 & .007 & .004 & .004 & .003 & .003 & .002 & .003 & .004 & .004 \\
\hline 17 & .006 & .005 & .006 & .007 & .004 & .004 & .003 & .003 & .002 & .004 & .004 & .004 \\
\hline 18 & .006 & .006 & .006 & .005 & .004 & .004 & .003 & .003 & .002 & .004 & .005 & .004 \\
\hline 19 & .007 & .006 & .006 & .004 & .004 & .004 & .003 & .003 & .002 & .004 & .005 & .004 \\
\hline 20 & .007 & .006 & .006 & .004 & .004 & .004 & .003 & .003 & .002 & .003 & .004 & .004 \\
\hline 21 & .008 & .005 & .007 & .004 & .004 & .004 & .003 & .003 & .002 & .003 & .004 & .004 \\
\hline 22 & .008 & .004 & .007 & .004 & .004 & .004 & .003 & .003 & .003 & .003 & .005 & .004 \\
\hline 23 & .007 & .004 & .006 & .004 & .004 & .004 & .004 & .002 & .003 & .004 & .005 & .004 \\
\hline 24 & .006 & .004 & .005 & .004 & .004 & .004 & .004 & .002 & .003 & .003 & .005 & .004 \\
\hline 25 & .006 & .004 & .005 & .004 & .004 & .004 & .004 & .002 & .002 & .003 & .004 & .004 \\
\hline 26 & .006 & .005 & .005 & .004 & .004 & .004 & .004 & .002 & .002 & .003 & .004 & .004 \\
\hline 27 & .006 & .005 & .005 & .004 & .005 & .004 & .004 & .002 & .002 & .003 & .004 & .004 \\
\hline 28 & .006 & .005 & .005 & .003 & .006 & .004 & .003 & .002 & .001 & .004 & .004 & .004 \\
\hline 29 & .006 & .005 & .005 & .004 & .007 & .003 & .003 & .002 & .001 & .004 & .004 & .005 \\
\hline 30 & .006 & .004 & .005 & .004 & ----- & .003 & .003 & .002 & .001 & .004 & .004 & .005 \\
\hline 31 & .006 & ----- & .006 & .004 & ----- & .003 & ----- & .002 & ------ & .004 & .004 & ----- \\
\hline Total & 0.202 & 0.153 & 0.168 & 0.198 & 0.122 & 0.145 & 0.095 & 0.075 & 0.065 & 0.095 & 0.116 & 0.122 \\
\hline Mean & .007 & .005 & .005 & .006 & .004 & .005 & .003 & .002 & .002 & .003 & .004 & .004 \\
\hline Max & .009 & .006 & .008 & .015 & .007 & .008 & .004 & .003 & .003 & .004 & .005 & .005 \\
\hline Min & .005 & .004 & .004 & .003 & .004 & .003 & .003 & .002 & .001 & .001 & .003 & .004 \\
\hline $\mathrm{Ac}-\mathrm{Ft}$ & .401 & .303 & .333 & .393 & .242 & .288 & .188 & .149 & .129 & .188 & .230 & .242 \\
\hline Cal Year & 1999 & Tota & & 263 & Mean & .006 & Max & .040 & Min & 0 & Ac-Ft & 4.49 \\
\hline Wtr Year & 2000 & Tota & & 556 & Mean & .004 & Max & .015 & Min & .001 & $\mathrm{Ac}-\mathrm{Ft}$ & 3.09 \\
\hline
\end{tabular}




\section{S002 Burning Ground Spring at TA-16}

Location. Lat 35'50'58", long 106 $20^{\prime} 17^{\prime \prime}, 150$ yds downstream from the SWSC line crossing of Cañon del Valle in Laboratory TA-16.

Gage. Data logger with $90^{\circ}$ weir. Elevation of gage is $7,420.8 \mathrm{ft}$ above National Geodetic Vertical Datum of 1929, from GPS survey.

Period of Record. October 1, 1996, to September 30, 2000.

Remarks. Water discharge records fair. This spring is in the Cañon del Valle drainage.

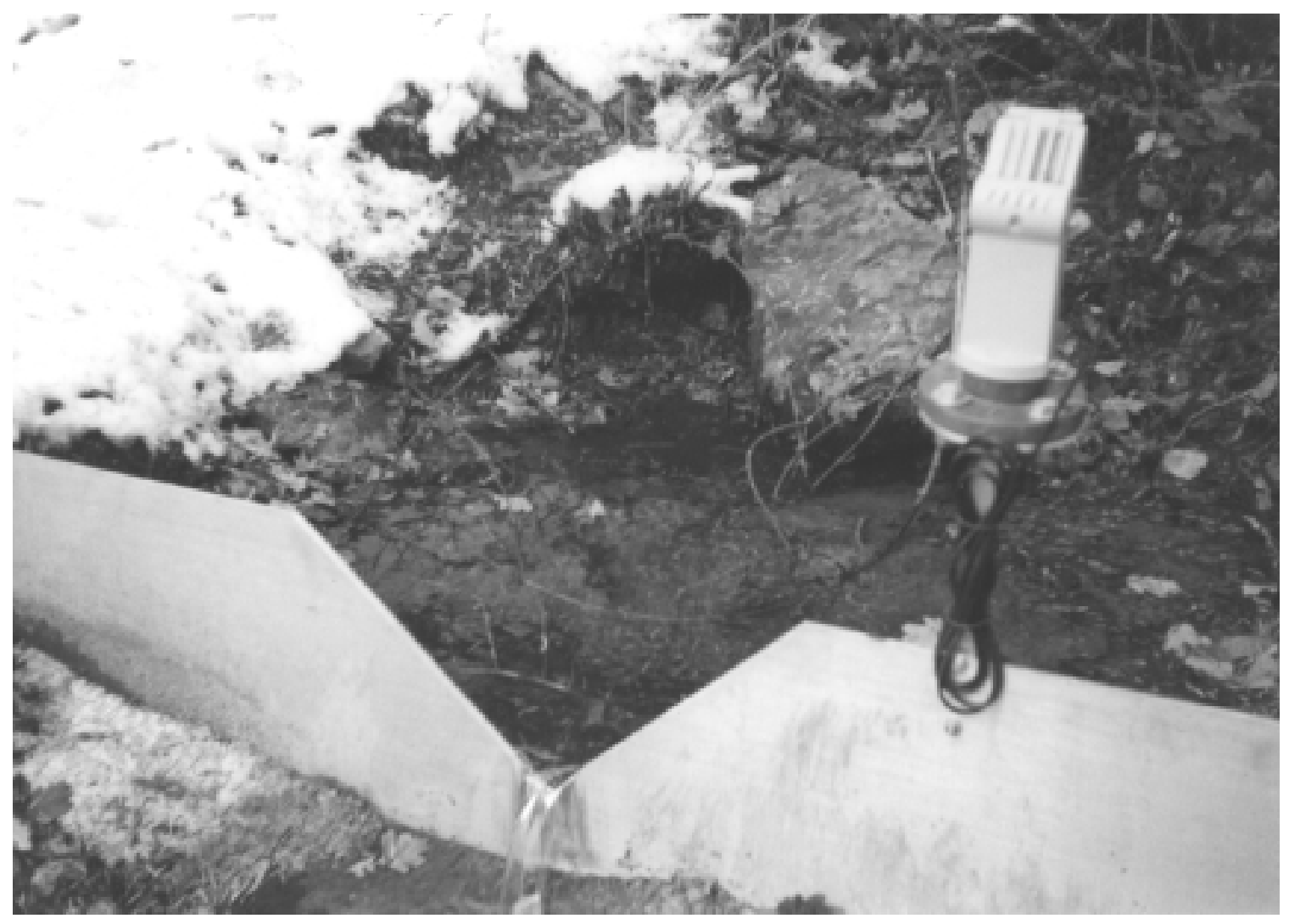




\section{S002 Burning Ground Spring at TA-16}

Daily Mean Discharge in Cubic Feet per Second

Water Year October 1999 to September 2000

\begin{tabular}{|c|c|c|c|c|c|c|c|c|c|c|c|c|}
\hline DAY & OCT & NOV & DEC & JAN & FEB & MAR & APR & MAY & JUN & JUL & AUG & SEP \\
\hline 1 & .017 & .017 & .015 & .015 & .017 & .016 & .011 & .010 & .008 & .008 & .009 & .009 \\
\hline 2 & .017 & .017 & .015 & .014 & .018 & .014 & .012 & .010 & .008 & .009 & .009 & .009 \\
\hline 3 & .018 & .017 & .017 & .014 & .017 & .014 & .012 & .010 & .009 & .009 & .009 & .008 \\
\hline 4 & .019 & .017 & .016 & .013 & .018 & .015 & .013 & .010 & .009 & .009 & .009 & .008 \\
\hline 5 & .021 & .017 & .016 & .014 & .017 & .015 & .013 & .010 & .008 & .009 & .009 & .008 \\
\hline 6 & .021 & .017 & .016 & .014 & .018 & .016 & .013 & .010 & .008 & .009 & .008 & .008 \\
\hline 7 & .020 & .017 & .015 & .014 & .017 & .015 & .013 & .010 & .008 & .009 & .009 & .009 \\
\hline 8 & .021 & .017 & .013 & .014 & .018 & .015 & .013 & .011 & .008 & .009 & .008 & .009 \\
\hline 9 & .023 & .017 & .013 & .015 & .018 & .015 & .013 & .010 & .008 & .009 & .008 & .008 \\
\hline 10 & .023 & .017 & .014 & .015 & .018 & .015 & .013 & .010 & .008 & .009 & .008 & .008 \\
\hline 11 & .028 & .017 & .014 & .015 & .018 & .014 & .013 & .010 & .008 & .009 & .008 & .008 \\
\hline 12 & .028 & .017 & .014 & .015 & .018 & .015 & .012 & .009 & .008 & .009 & .008 & .008 \\
\hline 13 & .029 & .017 & .015 & .015 & .019 & .015 & .013 & .010 & .009 & .009 & .009 & .008 \\
\hline 14 & .028 & .017 & .014 & .015 & .018 & .015 & .013 & .010 & .008 & .009 & .008 & .008 \\
\hline 15 & .027 & .016 & .014 & .015 & .018 & .015 & .014 & .008 & .009 & .009 & .008 & .008 \\
\hline 16 & .022 & .016 & .015 & .016 & .019 & .014 & .014 & .008 & .008 & .009 & .008 & .008 \\
\hline 17 & .023 & .017 & .015 & .015 & .018 & .015 & .013 & .008 & .008 & .009 & .009 & .009 \\
\hline 18 & .026 & .017 & .013 & .015 & .018 & .014 & .013 & .007 & .009 & .009 & .009 & .008 \\
\hline 19 & .028 & .016 & .013 & .015 & .016 & .013 & .013 & .007 & .009 & .009 & .009 & .008 \\
\hline 20 & .029 & .017 & .013 & .015 & .016 & .013 & .013 & .008 & .009 & .010 & .009 & .008 \\
\hline 21 & .028 & .017 & .013 & .018 & .015 & .012 & .012 & .009 & .008 & .010 & .009 & .009 \\
\hline 22 & .031 & .016 & .014 & .018 & .015 & .012 & .012 & .011 & .008 & .010 & .009 & .009 \\
\hline 23 & .030 & .016 & .016 & .018 & .015 & .012 & .010 & .010 & .009 & .010 & .010 & .008 \\
\hline 24 & .027 & .015 & .015 & .018 & .015 & .013 & .010 & .008 & .008 & .010 & .010 & .008 \\
\hline 25 & .027 & .015 & .014 & .018 & .014 & .013 & .011 & .008 & .008 & .010 & .010 & .008 \\
\hline 26 & .027 & .015 & .015 & .019 & .014 & .013 & .010 & .008 & .008 & .010 & .010 & .008 \\
\hline 27 & .023 & .016 & .015 & .017 & .015 & .013 & .010 & .008 & .008 & .009 & .009 & .008 \\
\hline 28 & .018 & .016 & .014 & .017 & .015 & .013 & .010 & .008 & .009 & .009 & .009 & .008 \\
\hline 29 & .018 & .015 & .015 & .017 & .015 & .013 & .010 & .008 & .008 & .009 & .009 & .008 \\
\hline 30 & .018 & .016 & .014 & .017 & ----- & .013 & .010 & .008 & .008 & .009 & .009 & .008 \\
\hline 31 & .017 & ----- & .015 & .017 & ----- & .012 & ----- & .008 & ------ & .009 & .009 & ----- \\
\hline Total & 0.732 & 0.494 & 0.450 & 0.487 & 0.487 & 0.432 & 0.362 & 0.280 & 0.249 & 0.285 & 0.274 & 0.247 \\
\hline Mean & .024 & .016 & .015 & .016 & .017 & .014 & .012 & .009 & .008 & .009 & .009 & .008 \\
\hline Max & .031 & .017 & .017 & .019 & .019 & .016 & .014 & .011 & .009 & .010 & .010 & .009 \\
\hline Min & .017 & .015 & .013 & .013 & .014 & .012 & .010 & .007 & .008 & .008 & .008 & .008 \\
\hline Ac-Ft & 1.45 & .980 & .893 & .966 & .966 & .857 & .718 & .555 & .494 & .565 & .543 & .490 \\
\hline Cal Year & 1999 & Tota & & & Mean & .105 & Max & 2.20 & Min & .010 & $\mathrm{Ac}-\mathrm{Ft}$ & 75.7 \\
\hline Wtr Year & 2000 & Tota & & & Mean & .013 & Max & .031 & Min & .007 & Ac-Ft & 9.48 \\
\hline
\end{tabular}




\section{S003 Martin Spring at TA-16}

Location. Lat $35^{\circ} 50^{\prime} 32^{\prime \prime}$, long $106^{\circ} 20^{\prime} 11^{\prime \prime}, 1 / 4$ mi south of building 344 in Laboratory TA-16.

Gage. Data logger with $90^{\circ}$ weir. Elevation of gage is $7,429.5 \mathrm{ft}$ above National Geodetic Vertical Datum of 1929, from GPS survey.

Period of Record. October 1, 1996, to September 30, 2000.

Remarks. Water discharge records fair. This spring is in the Water Canyon drainage.

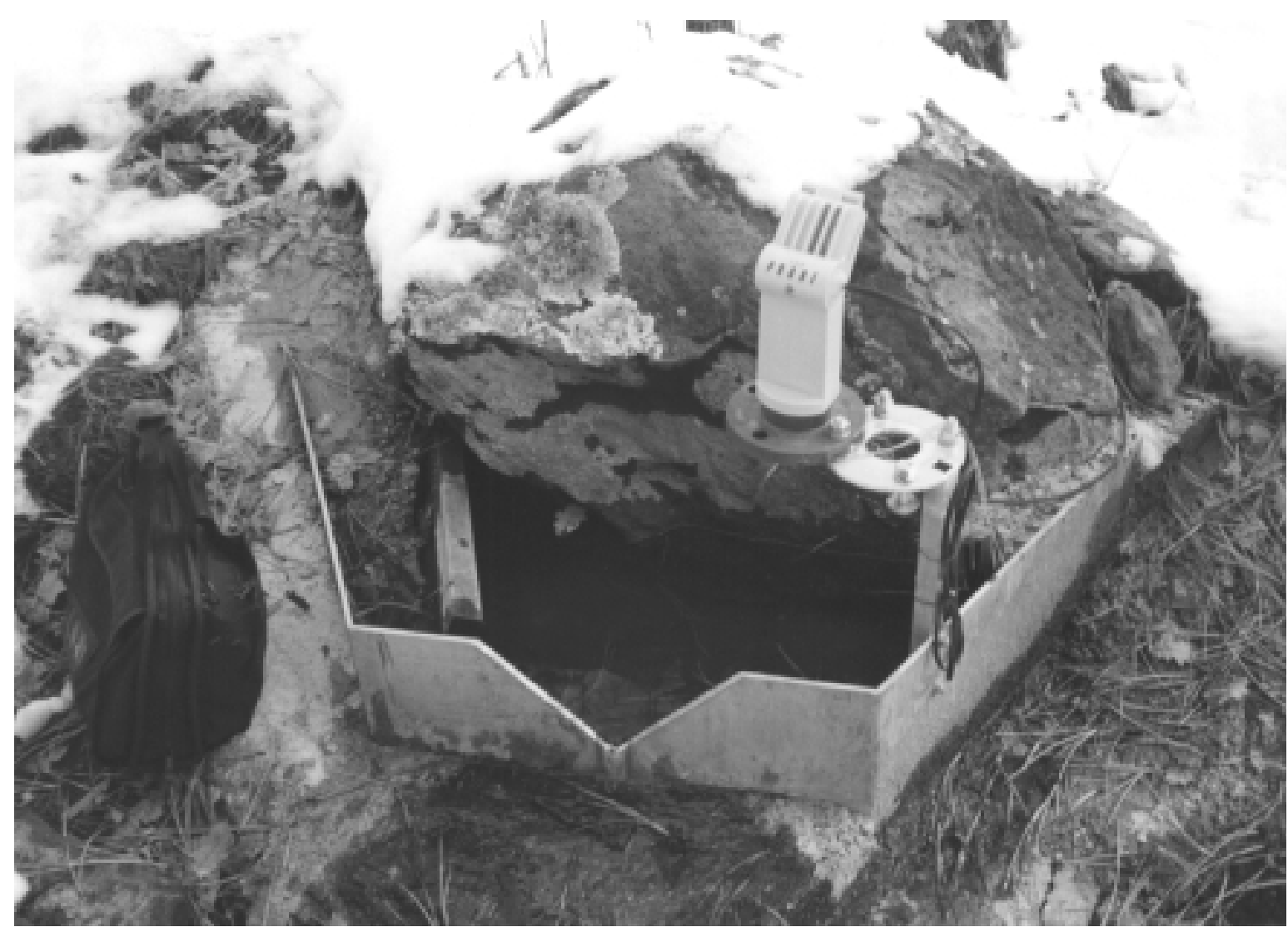


S003 Martin Spring at TA-16

Daily Mean Discharge in Cubic Feet per Second

Water Year October 1999 to September 2000

\begin{tabular}{|c|c|c|c|c|c|c|c|c|c|c|c|c|}
\hline DAY & OCT & NOV & DEC & JAN & FEB & MAR & APR & MAY & JUN & JUL & AUG & SEP \\
\hline 1 & .002 & .002 & .002 & .002 & .002 & .002 & .002 & .002 & .001 & .002 & .002 & .002 \\
\hline 2 & .002 & .002 & .002 & .002 & .002 & .001 & .002 & .002 & .002 & .002 & .002 & .001 \\
\hline 3 & .002 & .002 & .002 & .002 & .002 & .002 & .002 & .002 & .002 & .002 & .002 & .001 \\
\hline 4 & .002 & .002 & .002 & .002 & .002 & .002 & .002 & .002 & .002 & .002 & .002 & .001 \\
\hline 5 & .002 & .002 & .002 & .002 & .002 & .002 & .003 & .001 & .001 & .002 & .002 & .002 \\
\hline 6 & .002 & .002 & .002 & .001 & .002 & .002 & .002 & .001 & .001 & .002 & .002 & .001 \\
\hline 7 & .002 & .002 & .002 & .002 & .002 & .002 & .002 & .002 & .001 & .001 & .001 & .001 \\
\hline 8 & .002 & .002 & .002 & .002 & .002 & .002 & .002 & .002 & .002 & .002 & .002 & .001 \\
\hline 9 & .002 & .002 & .002 & .002 & .002 & .002 & .002 & .002 & .002 & .002 & .001 & .002 \\
\hline 10 & .002 & .002 & .002 & .002 & .002 & .002 & .002 & .002 & .002 & .002 & .001 & .001 \\
\hline 11 & .002 & .002 & .002 & .002 & .002 & .001 & .002 & .002 & .002 & .002 & .001 & .001 \\
\hline 12 & .002 & .002 & .002 & .002 & .002 & .001 & .002 & .001 & .002 & .002 & .001 & .002 \\
\hline 13 & .002 & .002 & .002 & .002 & .002 & .002 & .002 & .002 & .002 & .002 & .002 & .001 \\
\hline 14 & .002 & .002 & .002 & .002 & .002 & .002 & .002 & .002 & .002 & .002 & .002 & .002 \\
\hline 15 & .002 & .002 & .002 & .002 & .002 & .002 & .002 & .002 & .002 & .002 & .001 & .001 \\
\hline 16 & .002 & .002 & .002 & .002 & .002 & .001 & .002 & .002 & .002 & .002 & .001 & .001 \\
\hline 17 & .002 & .002 & .002 & .002 & .002 & .001 & .002 & .002 & .001 & .001 & .002 & .001 \\
\hline 18 & .002 & .002 & .002 & .002 & .002 & .001 & .002 & .002 & .001 & .001 & .002 & .001 \\
\hline 19 & .002 & .002 & .002 & .002 & .002 & .001 & .002 & .002 & .002 & .002 & .002 & .001 \\
\hline 20 & .002 & .002 & .002 & .002 & .002 & .002 & .002 & .002 & .002 & .002 & .002 & .001 \\
\hline 21 & .002 & .002 & .001 & .002 & .002 & .002 & .002 & .002 & .002 & .002 & .001 & .002 \\
\hline 22 & .002 & .002 & .002 & .002 & .002 & .002 & .002 & .002 & .001 & .002 & .001 & .002 \\
\hline 23 & .002 & .002 & .002 & .002 & .002 & .002 & .002 & .002 & .002 & .001 & .001 & .001 \\
\hline 24 & .002 & .002 & .002 & .002 & .002 & .002 & .001 & .001 & .002 & .001 & .001 & .002 \\
\hline 25 & .002 & .002 & .002 & .001 & .002 & .002 & .001 & .002 & .001 & .001 & .001 & .001 \\
\hline 26 & .002 & .002 & .002 & .001 & .002 & .002 & .001 & .002 & .002 & .001 & .002 & .001 \\
\hline 27 & .002 & .002 & .002 & .002 & .002 & .002 & .001 & .002 & .002 & .001 & .002 & .001 \\
\hline 28 & .002 & .002 & .002 & .002 & .002 & .002 & .002 & .002 & .002 & .002 & .002 & .001 \\
\hline 29 & .002 & .002 & .002 & .001 & .002 & .002 & .002 & .002 & .002 & .002 & .002 & .001 \\
\hline 30 & .002 & .002 & .002 & .001 & ----- & .002 & .002 & .002 & .002 & .002 & .002 & .001 \\
\hline 31 & .002 & ----- & .002 & .002 & ---- & .002 & $----{ }_{-}$ & .002 & $----\cdot$ & .002 & .002 & ----- \\
\hline Total & 0.062 & 0.060 & 0.061 & 0.057 & 0.058 & 0.055 & 0.057 & 0.058 & 0.052 & 0.054 & 0.050 & 0.038 \\
\hline Mean & .002 & .002 & .002 & .002 & .002 & .002 & .002 & .002 & .002 & .002 & .002 & .001 \\
\hline $\operatorname{Max}$ & .002 & .002 & .002 & .002 & .002 & .002 & .003 & .002 & .002 & .002 & .002 & .002 \\
\hline Min & .002 & .002 & .001 & .001 & .002 & .001 & .001 & .001 & .001 & .001 & .001 & .001 \\
\hline $\mathrm{Ac}-\mathrm{Ft}$ & .123 & .119 & .121 & .113 & .115 & .109 & .113 & .115 & .103 & .107 & .099 & .075 \\
\hline Cal Year & 1999 & Tota & & 213 & Mean & .001 & $\operatorname{Max}$ & .030 & Min & 0 & $\mathrm{Ac}-\mathrm{Ft}$ & .422 \\
\hline Wtr Year & 2000 & Tota & & 662 & Mean & .002 & $\operatorname{Max}$ & .003 & Min & .001 & $\mathrm{Ac}-\mathrm{Ft}$ & 1.31 \\
\hline
\end{tabular}


This report has been reproduced directly from the best available copy. It is available electronically on the Web (http://www.doe.gov/bridge).

Copies are available for sale to U.S. Department of Energy employees and contractors from-

Office of Scientific and Technical Information

P.O. Box 62

Oak Ridge, TN 37831

(423) 576-8401

Copies are available for sale to the public from-

National Technical Information Service

U.S. Department of Commerce

5285 Port Royal Road

Springfield, VA 22616

(800) 553-6847 


\section{Los Alamos \\ NATIONAL LABORATORY \\ Los Alamos, New Mexico 87545}

